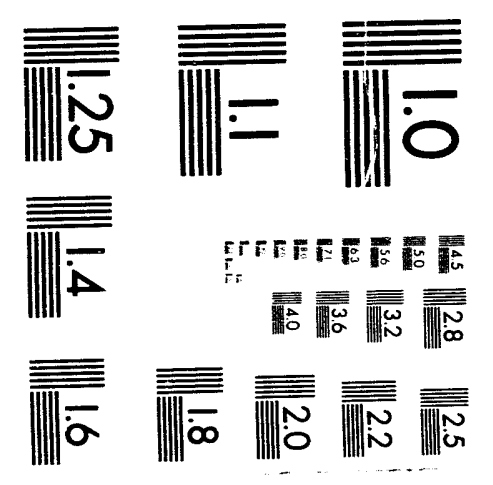



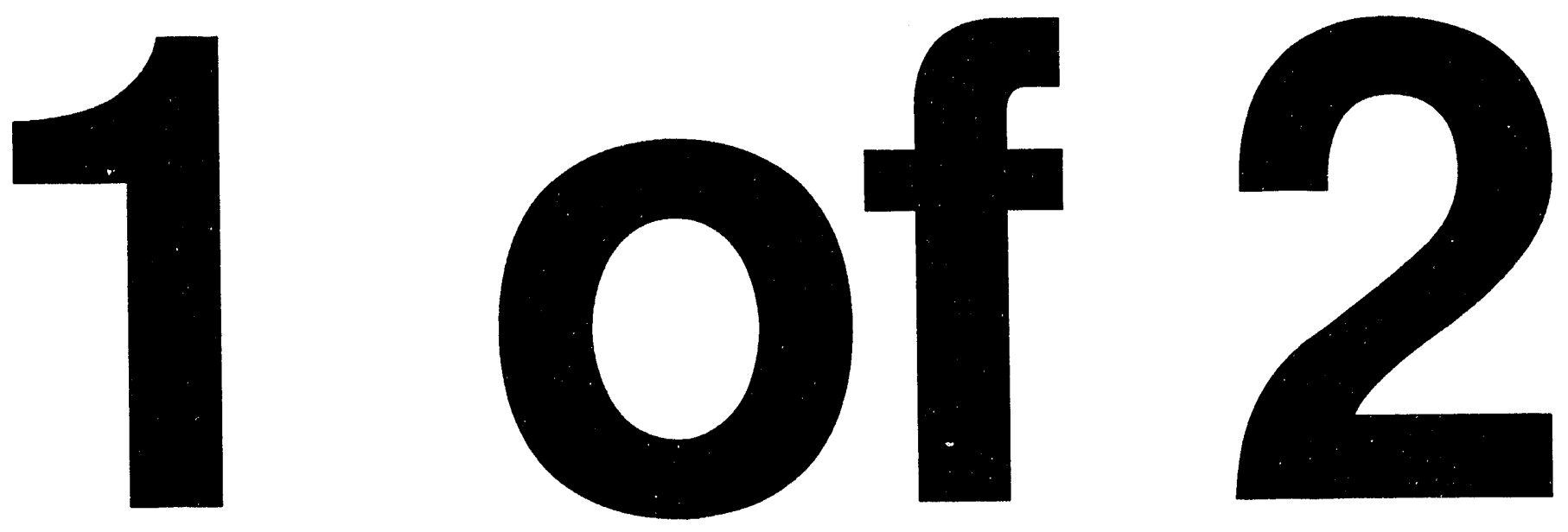


\title{
Vacuum System Pump Down Analysis
}

\author{
D. R. Rohrdanz
}

Date Published

August 1990

Prepared for the U.S. Deparment of Energy Assistant Secretary for Nuclear Energy

\footnotetext{
(20) Westinghouse Hanford Company Richland, Washington 99352

Hanford Operations and Engineering Contractor for the U.S. Department of Energy under Contract DE-AC06-87RL10930
}

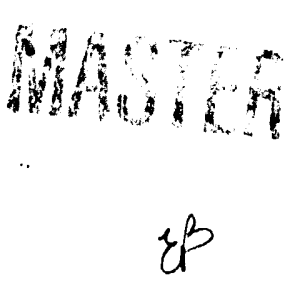




\section{DISCLAIMER}

This report was prepared as an account of work sponsored by an agency of the United States Government. Neither the United States Government nor any agency thereof, nor any of their employees, nor any of their contractors, subcontractors or their employees, makes any warranty, express or implied, or assumes any legal liability or responsibility for the accuracy, completeness, or any third party's use of the results of such use of any information, apparatus, product, or process disclosed, of represents that its use would not inifinge privately owned rights. Reference herein to any specific commercial product, process, or service by trade name, trademark, manufacturer, or otherwise, does not necessarily constiture or imply its endorsement, recommendation, or favoring by the United States Government of any agency thereof or its contractors or subcontractors. The views and opinions of authors expressed herein do nol necessarily state or reflect those of the United States Government or any agency thereof.

Printed in the Unitod States of Ameriea

DISCLM-2.CHP (7-90) 
NORCUS

SP-100 Vacuum System

Denise S Fohrdanz

July 27,1990

\section{INTRODUCTION}

My assignment on the SP-100 Vacuum Vessel Vacuum System Team was to perform a transient pump down analysis for the vacuum vessel that will house the SP. 100 reactor during testing. Pump down time was calculated for air and helium.

\section{PUMPS}

The baseline vacuum pumping system configuration consisted of a rotary piston or roots blower pump in parallel with one or two turbomolecular pumps. The rotary piston and roots blower pumps were used in the viscous and transitional flow regimes. The turbomolecular pumps operate in the molecular flow regime.

Pumps were evaluated on a pumping speed vs inlet pressure basis. For the rotary piston/roots blower pumps, the same pump curve were used for both air and helium according to manufacturer's recommendation. Air and helium were plotted separately for the turbomolecular pumps.

\section{CONDUCTANCE}

The conductance elements (pipes, elbows, bends, etc.) for the roughing line connecting the rotary piston/roots blower pump to the vessel were input to * VACTRAN (VACTRAN A Vacuum System Design Tool, version 2.0) based on configurations provided and the conductance was calculated by the program.

Conductance for the turbomolecular pumps was calculated based on configurations provided. The tube-only conductance for the ducting connecting the turbomolecular pump to the vessel was calculated using the equations for molecular flow (Santeler, 338-342).

\section{GAS LOAD}

Bake-out, heating the vessel for a period of 15 days, will accelerate outgassing and vaporize any water collected on the interior wall of the vessel. Following bake-out, this analys is assumed a steady outgassing rate of 1.1E-06 torr-liters/second.

*VACTRAN is a trademark of Professional Engineering Computations 


\section{SP-100 Vacuum System \\ Denise S Rohrdanz \\ July 27, 1990}

PUMP DOWN

The pump down time is the amount of time it takes to achieve a target pressure for any given system model. Eight systems were modelled; 4-air and 4-helium. Pump curves were plotted for each case assuming duct sizes of 4,6 and 8 inches and the results tabulated. The specified maximum time allowed for pump down is 24 hours.

\section{ACCURACY}

A subsequent analysis using the Vacuum System Design (VSO) (Vacuum System Design, version 2.2-2) software confirmed the results obtained from the VACTRAN program.

The necessary pump curve constants were calculated from the pump curves plotted on the VACTRAN system for air and helium and input to VSO (Vacuum System Design, 33).

Conductance was calculated by VSD (Vacuum System Design, version 2.2-2) using a rotary piston pump and 2-turbopumps with 4 -inch ducting. The results of the two programs were compared and found to be in agreement. Therefore, the results obtained from VACTRAN were considered valid and reasonable.

\section{CONCLUSION}

For all cases the proposed vacuum system will be able to pump down the vessel within the required time. The use of a larger rotary piston pump (DU0250) improves the pump down time by 35 minutes and therefore should be considered. The 6 -inch duct for the roughing line is optimal, however, because all cases are well below the 24 hour time frame, the 4 -inch duct is sufficient. The use of the single turbomolecular pump during pump down is sufficient. A pump down with helium in the vessel and a helium inleakage delays the time to achieve the base pressure marginally and is acceptable. 

3

$\begin{array}{llll} & & 0 & 0 \\ 0 & & 0 & 0 \\ 0 & & 0 & c \\ 0 & & 0 & c \\ 0 & & z & 3 \\ 0 & 0 & z & \end{array}$

ग्रे $>$

$z$ is

$\vec{r} \vec{m}$

is 3

$m$ 
TABLE OF CONTENTS

Vacuum System

Pump Down Analysis

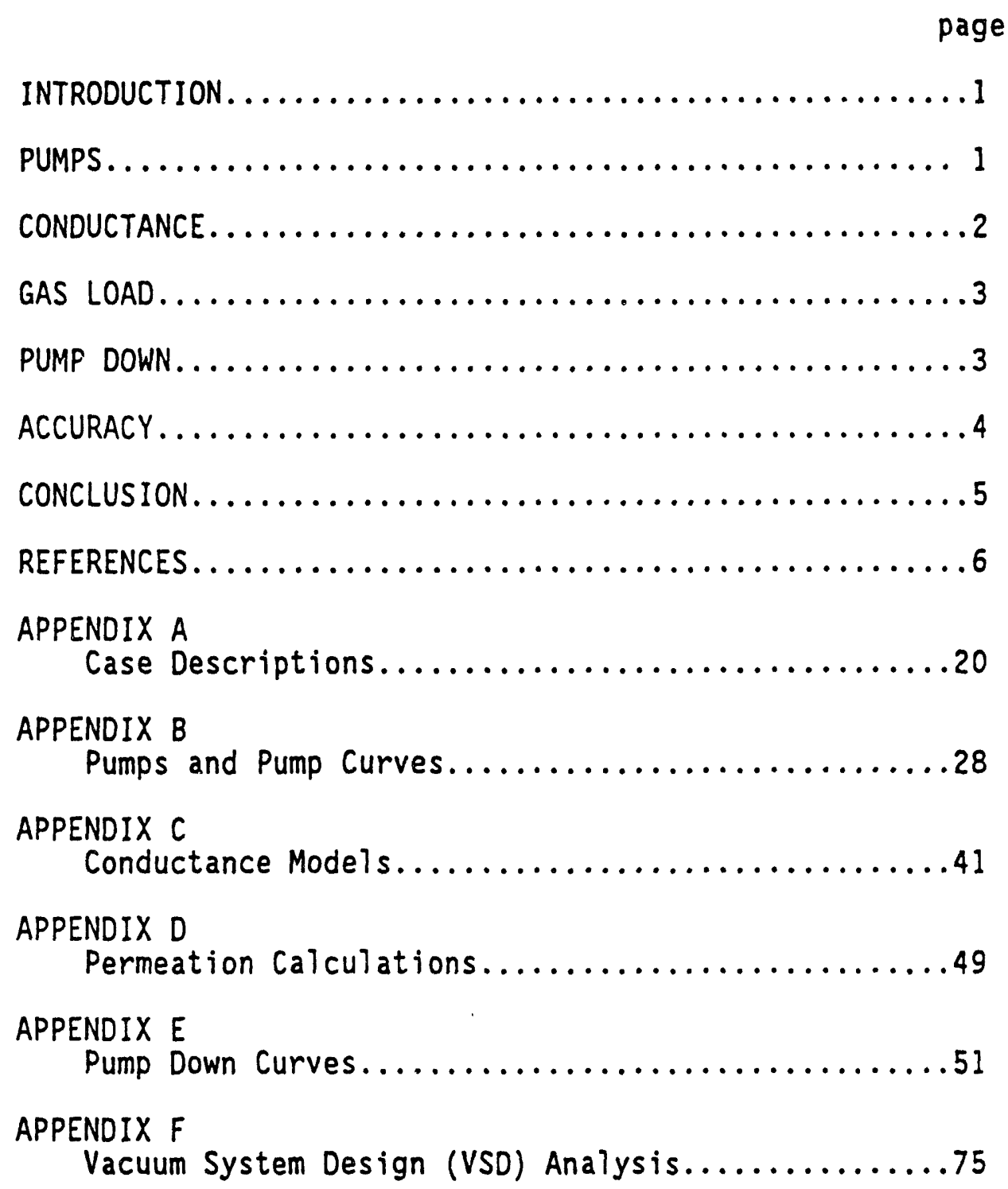




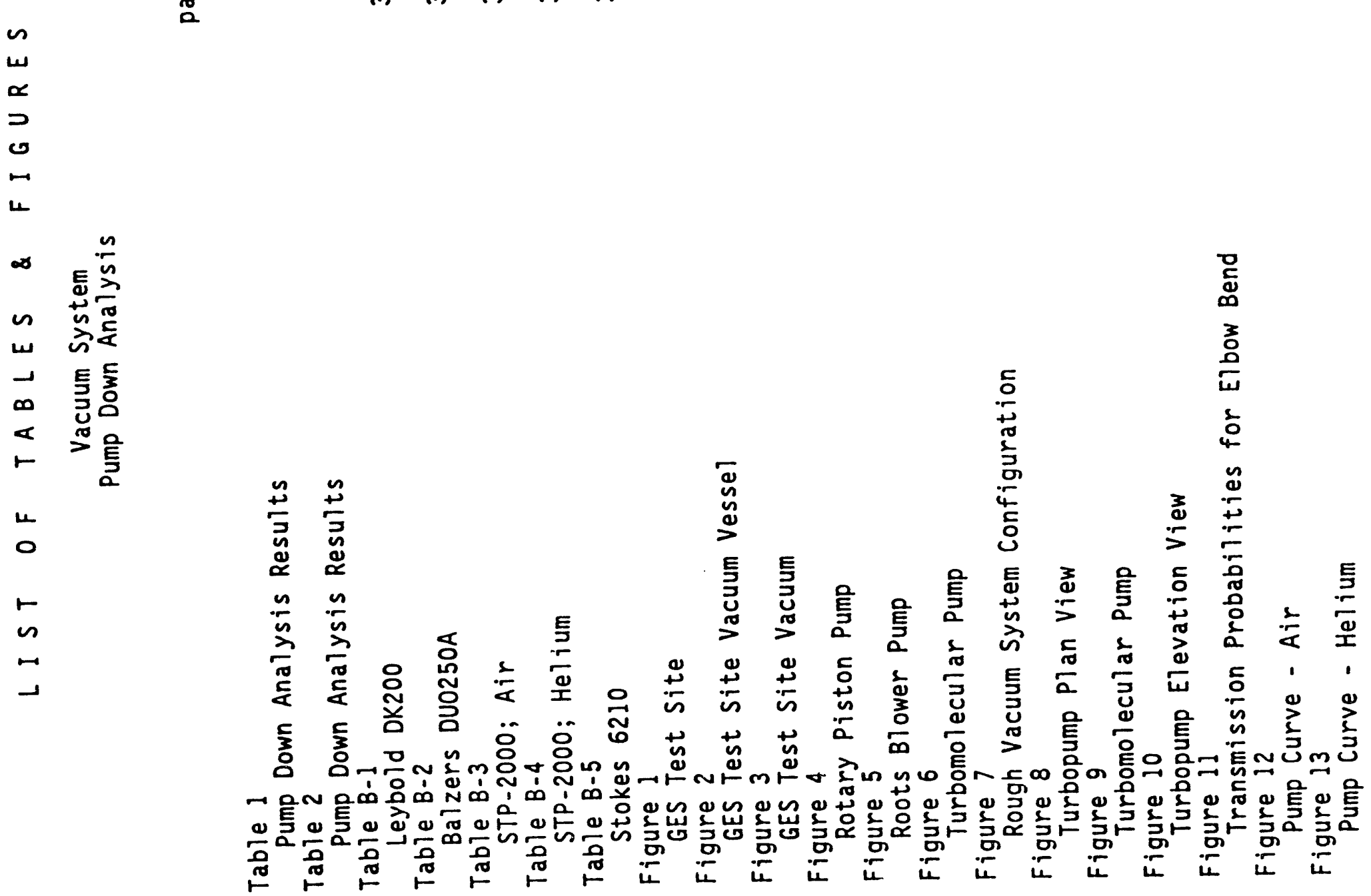


LIST OF TA (continued)

Vacuum System

Pump Down Analysis

page

Appendix B

Figure $B-1$

DK200 Pump Curve - Air/Helium 32

Figure $\mathrm{B}-2$

DK200 Manufacturers Pump Curve-Air/Helium 33

Figure $B-3$ DU0250A Pump Curve-Air/Helium 34

Figure $B-4$ DU0250A Manufacturers Pump Curve-Air/Helium 35

Figure $B-5$ STP2000 Pump Curve - Air 36

Figure $B-6$ STP2000 Pump Curve - Helium 37

Figure $B-7$ STP2000 Manufacturers Pump Curve - Air/Helium 38

Figure $B-8$ Stokes 6210 Pump Curve - Air/Helium 39

Figure $B-9$ Stokes 6210 Manufacturers Pump Curve - Air/Helium 40

Appendix $C$

Figure $C-1 A$ Rough Pump Conductance - Air (4-inch duct) 43

Figure $C-1 B$ Rough Pump Conductance - Air (6-inch duct) 44

Figure $\mathrm{C}-1 \mathrm{C}$ Rough Pump Conductance - Air (8-inch duct) 45

Figure $C-2 A$ Rough Pump Conductance - Helium (4-inch duct) 46

Figure $C-2 B$ Rough Pump Conductance - Helium (6-inch duct) 47

Figure $\mathrm{C}-2 \mathrm{C}$ Rough Pump Conductance - Helium (8-inch duct) 48

Appendix E

Figure $E-1 A$ Case IA Pump Down Curve - Air (4-inch duct) 51

Figure $E-1 B$ Case IB Pump Down Curve - Air (6-inch duct) 52

Figure $E-1 C$

Case IC Pump Down Curve - Air (8-inch duct) 53

Figure $E-2 A$

Case 2A Pump Down Curve - Air (4-inch duct) 


\section{LIST OF TABLES \& FIGURES (continued)}

Vacuum System

Pump Down Analysis

Appendix $E$ (cont'd)

Figure $E-2 B$

Case 2B Pump Down Curve - Air (6-inch duct) 55

Figure $E-2 C$

Case 2C Pump Down Curve - Air (8-inch duct) 56

Figure $E-3 A$ Case $3 A$ Pump Down Curve - Air (4-inch duct) 57

Figure $E-3 B$

Case 3B Pump Down Curve - Air (6-inch duct) 58

Figure $E-3 C$

Case 3C Pump Down Curve - Air (8-inch duct) 59

Figure $E-4 A$

Case 4A Pump Down Curve - Air (4-inch duct) 60

Figure $E-4 B$

Case $4 B$ Pump Down Curve - Air (6-inch duct) 61

Figure $E-4 C$

Case 4C Pump Down Curve - Air (8-inch duct) 62

Figure $E-5 A$

Case 5A Pump Down Curve - Helium (4-inch duct) 63

Figure $E-5 B$

Case 5B Pump Down Curve - Helium (6-inch duct) 64

Figure $E-5 C$

Case 5C Pump Down Curve - Helium (8-inch duct) 65

Figure $E-6 A$

Case 6A Pump Down Curve - Helium (4-inch duct) 66

Figure $E-6 B$

Case 6B Pump Down Curve - Helium (6-inch duct) 67

Figure $E-6 C$

Case 6C Pump Down Curve - Helium (8-inch duct) 68

Figure $E-7 A$

Case 7A Pump Down Curve - Helium (4-inch duct) 69

Figure $E-7 B$

Case 7B Pump Down Curve - Helium (6-inch duct) 70

Figure $E-7 C$

Case 7C Pump Down Curve - Helium (8-inch duct) 71

Figure E-8A

Case 8A Pump Down Curve - Helium (4-inch duct) 72

Figure $E-8 B$

Case 8B Pump Down Curve - Helium (6-inch duct) 73

Figure $E-8 C$

Case 8C Pump Down Curve - Helium (8-inch duct) 74 


\section{INTRODUCTION}

The following is a transient pump down analysis for the vacuum vessel, shown in Figure 2, that will house a reactor during ground testing. The test facilities will be designed to simulate space by providing a vacuum environment for the reactor test assembly. The yessel volume is 34,130 liters with an inside surface area of $781,638 \mathrm{~cm}^{2}$.

The baseline vacuum pumping system configuration consists of a rotary piston or roots blower pump in parallel with one or two turbomolecular pumps.

Figure 3 is a schematic of the baseline vacuum system. The rotary piston/roots blower pump is assumed to be turned on at 760 torr and will operate until a pressure of 8E-03 torr is achieved. Once 8E-03 torr is reached the turbomolecular pump(s) is turned on and the rotary piston/roots blower pump turned off. The target pressure for this analys is is $1 E-8$ torr.

The vessel is initially assumed to contain air from the atmosphere during assembly. Helium will be used to backfill the vessel to avoid oxygen entering the vessel and reacting with the test assembly refractory metals. Therefore, pump down time is also calculated for helium.

\section{PUMPS}

The rotary piston (Figure 4 ) and roots blower (Figure 5) pumps are used in the viscous and transitional flow regimes. Rotary piston pumps normally operate at a speed of 400-500 rpm but can be as low as $300 \mathrm{rpm}$ or as high as. $1200 \mathrm{rpm}$. (0'Hanlon, 167-168) The rotors on the roots blower pump are synchronized and rotate in opposite directions at speeds ranging from 3000 to $3500 \mathrm{rpm}$ (O'Hanion, 169-170). The rotary piston pumps modelied are the Leybold DK200 and the Balzers DU0250A. The roots blower modelled is the Stokes 6210.

The turbomolecular pumps (Figure 6) (0-Hanlon, 174) operate in the molecular flow regime. If allowed to operate with the rear blades in viscous or transitional flow the rotor will be subjected to additional torque due to the viscous drag. This results in loss of pumping speed. The turbomolecular pump modelled is a Seiko STP2000, a turbomolecular pump with magnetic Tevitated bearings.

Pumps are evaluated on a pumping speed vs inlet pressure basis as show'n in Appentix $B$. Data from the manufacturers' pump performance curve is input to VACTRAN (VACTRAN A Vacuum System Design Tool, version 2.0) and plotted. For the rotary piston/roots blower pumps, the same pump curve is used for

*VACTRAN is a trademark of Professional Engineering Computations 
both air and helium according to manufacturer's recommendation. Air and helium are plotted separately for the turbomolecular pumps.

\section{CONDUCTANCE}

Flow in the roughing line connecting the rotary piston/roots blower pump to the vessel is assumed to be in the viscous or transitional flow regimes and is therefore a function of pressure. The conductance elements (pipes, elbows, bends, etc.) are input to VACTRAN (VACTRAN A Vacuum System Design Tool, version 2.0) based on the configuration shown in Figure 7 and the conductance is calculated by the program.

In the molecular flow regime, conductance, at a given temperature, is proportional to the molecular weight of the gas species. As stated above, the turbomolecular pumps are assumed to be in molecular flow. Conductance is calculated based on the configuration shown in Figures 8,9 and 10 . The tube-only conductance for the ducting connecting the turbomolecular pump to the vessel is calculated using the equations for molecular flow (Santeler, 338-342).

$$
\begin{aligned}
C_{t}=[\alpha /(1-\alpha)] C_{o m} \\
\text { where: } \begin{aligned}
& C_{t}=\text { tube-only conductance, iters } / \mathrm{sec} \\
& \alpha=\text { transmission probability of pump duct } \\
& \text { and, } C_{\text {om }}=11.43(T / M) \cdot 5 \mathrm{a}^{2}
\end{aligned} \\
\text { where: } \begin{aligned}
T & =\text { temperature }(\mathrm{K}) \\
M & =\text { molecular weight } \\
\mathrm{a} & =\text { radius of pump duct, } \mathrm{cm}
\end{aligned}
\end{aligned}
$$

Transmission probability is taken from Figure 11 (0'Hanlon, 33). Figures 8 and 9 show the dimensions for the values $A$ and $B$ respectively.

For air at $366 \mathrm{~K}$, the system operating temperature, and transmission probability of .24, the conductance for the turbomolecular pumps is:

$$
\begin{aligned}
& C_{t}=[\alpha /(1-\alpha)]\left[11.43(T / M) \cdot 5 \mathrm{a}^{2}\right] \\
& C_{t}=[.24 /(1-.24)]\left[11.43(366 / 28) \cdot 513.06^{2}\right] \\
& C_{t}=2226 \mathrm{l} / \mathrm{s}, \text { Air }
\end{aligned}
$$


Vacuum System Analysis

Denise S Rohrdanz

July 19, 1990

Under the same conditions, the conductance for helium is:

$$
\begin{aligned}
& c_{t}=[.24 /(1-.24)]\left[11.43(366 / 4) \cdot 5_{\left.13.06^{2}\right]}\right. \\
& c_{t}=5889 \mathrm{l} / \mathrm{s}
\end{aligned}
$$

Conductance models are defined in Appendix $C$.

\section{GAS LOAD}

The gas load is defined as the rate of gas introduction into the vacuum chamber in units of pressure-volume/time. The sources of gas to the chamber are material outgassing, pelmeation, leaks and backstreaming.

Outgassing by diffusion and desorption is the release of gases trapped in the vessel material during fabrication as well as gas and vapor adsorbed on the interior walls from the atmosphere after assembly. The release of these gases varies with time, temperature and vacuum conditions.

At equilibrium, permeation behaves as a slow leak. Gases collect on the outer wall of the vessel and work their way through the vessel material to the inner wall to be desorbed into the vessel. Permeation through stainless steel is predominantiy hydrogen. Permeation in this case, calculated in Appendix $D$, is negligible compared to vessel leaks through flanges, etc. Leaks through the vessel flanges and mechanical penetration is specified at 1.1E-6 torr-liters/sec as a design limit.

Backstreaming is that amount of gas which flows back into the vessel from the pump. This may also include pump oil if proper precautions are not taken such as sorption traps and gas purges. 0il backstreaming can be eliminated by using 0il-free pumps such as the Seiko STP2000 turbomolecular pump.

Bake-out, heating the vessel at $423 \mathrm{~K}$ for a period of 15 days, will accelerate outgassing due to diffusion and desorption as well as vaporize any water collected on the interior wall of the vessel. Once bake-out is complete, the initial volume of gas is pumped and the outgassing load is depleted, this analysis assumes a steady outgassing rate of $1.1 \mathrm{E}-06$ torr-liters/second.

\section{PUMP DOWN}

The pump down time is the amount of time it takes to go from an initial pressure of 760 torr at start-up to a final pressure of $10^{-8}$ torr for any given system model. Appendix A contains descriptions of eight system models; 4-air and 4-helium. In each case the gas load is assumed to be 1.1E-6 torrliters/second. Appendix E contains all pump down curves for each case. Each case has three curves marked $A, B$ and $C$ corresponding to duct sizes of 
Vacuum System Analysis

Denise S Rohrdanz

July 19, 1990

4-inch, 6 -inch and 8 -inch respectively. The results are 1 isted in Table 1 below. The specified maximum time allowed for pump down is 24 hours.

\section{TABLE 1}

Pump Down Analysis Results

\section{Case Description}

No. AIR:

$\begin{array}{ll}1 & \text { DK200+2-STP2000 } \\ 2 & \text { DUO250+2-STP2000 } \\ 3 & \text { DK200+1-STP2000 } \\ 4 & \text { STOKES } 6210+2-\text { STP2000 }\end{array}$

HELIUM:

$\begin{array}{ll}5 & \text { DK200+2-STP2000 } \\ 6 & \text { DUO250+2-STP2000 } \\ 7 & \text { DK200+1-STP2000 } \\ 8 & \text { STOKES } 6210+2-\text { STP } 2000\end{array}$

\section{4-inch duct}

A

$2 \mathrm{hr}-40 \mathrm{~min}$

$2 \mathrm{hr}-05 \mathrm{~min}$

$2 \mathrm{hr}-45 \mathrm{~min}$

$2 \mathrm{hr}-20 \min$

A

$2 \mathrm{hr}-44 \min$

$2 \mathrm{hr}-08 \mathrm{~min}$

$2 \mathrm{hr}-46$ min

$2 \mathrm{hr}-14 \min$

\section{Pump Down Time}

6-inch duct 8-inch duct

\author{
$B$
}

$2 \mathrm{hr}-20 \mathrm{~min}$

$1 \mathrm{hr}-45 \mathrm{~min}$

$2 \mathrm{hr}-24 \mathrm{~min}$

$2 \mathrm{hr}-03 \mathrm{~min}$

B

$2 \mathrm{hr}-25 \mathrm{~min}$

$1 \mathrm{hr}-50 \mathrm{~min}$

$2 \mathrm{hr}-28 \mathrm{~min}$

$2 \mathrm{hr}-00 \mathrm{~min}$ $c$

$2 \mathrm{hr}-18 \mathrm{~min}$

$1 \mathrm{hr}-41 \mathrm{~min}$

$2 \mathrm{hr}-20 \mathrm{~min}$

$1 \mathrm{hr}-59 \mathrm{~min}$

C

\section{ACCURACY}

A subsequent analysis using the Vacuum System Design (VSD) (Vacuum System Design, version 2.2-2) software confirms the results shown above. The model used is the same as for case $1 \mathrm{~A}$ above with a Balzers DUO060A as a backing pump for the turbomolecular pump. Appendix $F$ contains the pump down analys is for this study.

The necessary pump curve constants (given below) are calculated from the pump curves plotted on the VACTRAN system for air and helium (Figures 12 and 13, respectively) and input to VSD (Vacuum System Design, 33).

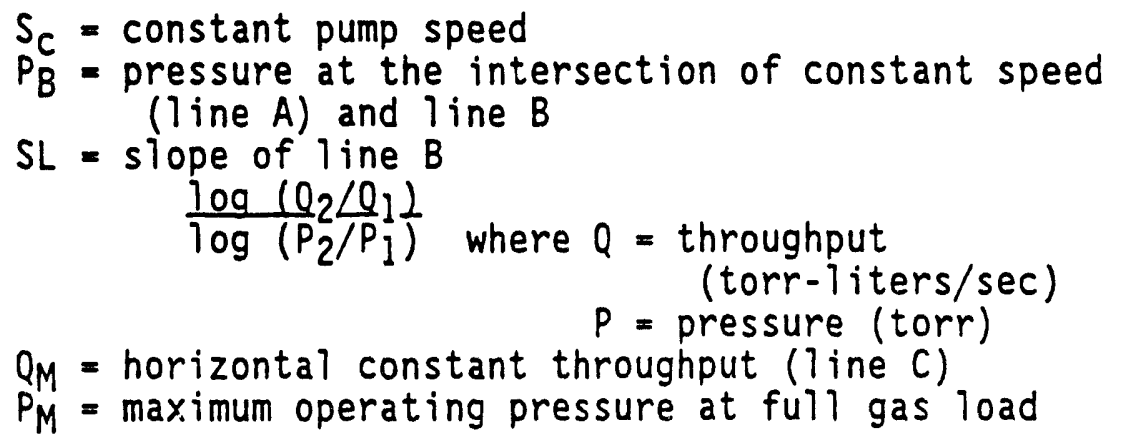


Vacuum System Analysis

Denise S Rohrdanz

July 19,1990

AIR:

$S L=\frac{\log (3 / 1)}{\log (4.3 E-3 / 8 E-4)}$
$S L=.653$
$P_{B}=8 E-4$ torr
$S_{C}=19201 / \mathrm{s}$
$Q_{m}=3$ torr-7iters $/ \mathrm{sec}$
HELIUM:

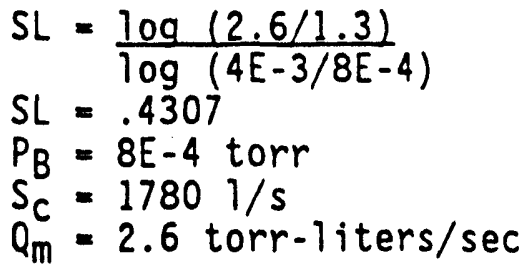

Conductance is calculated by VSD (Vacuum System Design, version 2.2-2) using the same configuration as case $1 \mathrm{~A}$ above. The vessel is assumed to contain air. The gas load, due to inleakage, is assumed to be $1.1 \mathrm{E}-06$ as in the previous analysis. A comparison of the results to those of case $1 \mathrm{~A}$ using VACTRAN is given in Table 2 below.

TABLE 2

Pump Down Analysis Results

Case Description

Air:

DK200 + 2-:STP2000
Pump Down Time VACTRAN $\quad \underline{\text { VSD }}$

$2 \mathrm{hr}-40 \min 2 \mathrm{hr}-49 \min$

\section{CONCLUSION}

A review of Table 1 reveals that for all cases the proposed vacuum system will be able to pump down the vessel within the required time of 24 hours. The use of a larger rotary piston pump (DU0250) improves the pump down time by 35 minutes and therefore should be considered. The 6 -inch duct for the roughing line is optimal, however, because all cases are well below the 24 hour time frame, the 4 -inch duct is sufficient. The use of the single turbomolecular pump during pump down is sufficient. A pump down with helium in the vessel and a helium inleakage delays the time to achieve the base pressure marginally and is acceptable.

A review of the results in Table 2 reveals that the two programs, VACTRAN and VSD, are in agreement. Therefore, the results obtained from VACTRAN are valid and reasonable. 
Vacuum System Analysis

Denise S Rohrdanz

July 19, 1990

\section{REFERENCES}

VACTRAN A Vacuum System Design Tool, Version 2.0, Professional Engineering Computations, 1990.

Vacuum System Desian, Version 2.2-2, Process Applications, Inc., 1987.

Holkeboer, David H., Jones, Donald W., Pagano, Frank., Santeler, Donald J., Vacuum Technology and Space Simulation, Scientific and Technical Information Division, National Aeronautics and Space Administration, Washington D.C., 1966, PP 191-192.

O'Hanton, John F., A Users Guide to Vacuum Technology, John Wiley and Sons, New York, 1980, pp 33, 167-168, 169-170, 174.

Article by D. J. Santeler, New Concepts in Molecular Gas Flow, Journal of Vacuum Science Technology, May, June 1986, pp 338-342.

$W$. Kass and W. Andrzewski, The Permeation and Diffusion of $\mathrm{H}_{2}$ and $\mathrm{D}_{2}$ in 309 Stainless Steel, SC-DR-72-1036, Sandia Laboratories, 1972.

W. G. Perkins, Permeation and Outgassing of Vacuum Materials, SC-DC-72-1321, Sandia Laboratories, 1972. 
GES TEST SITE

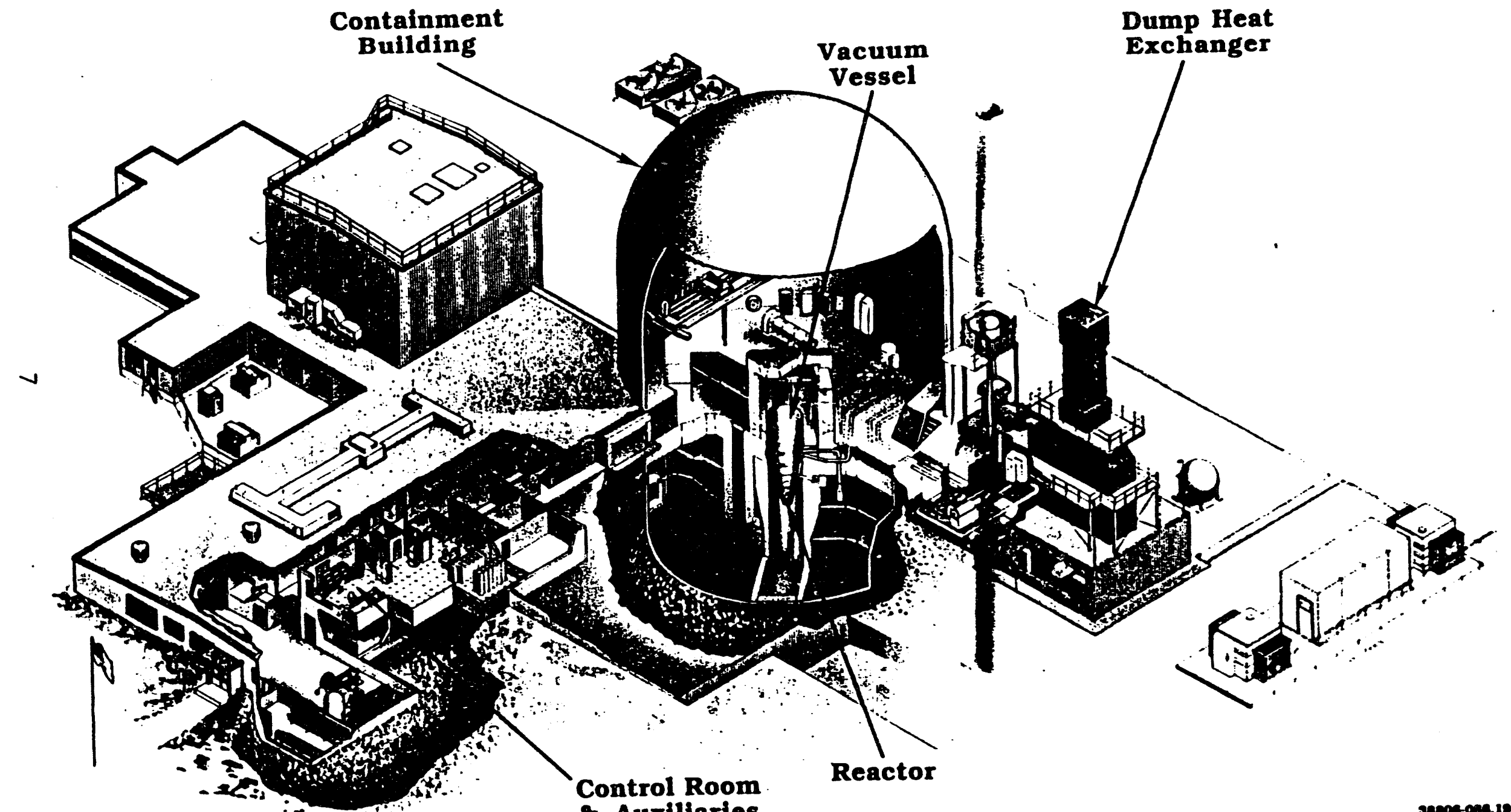




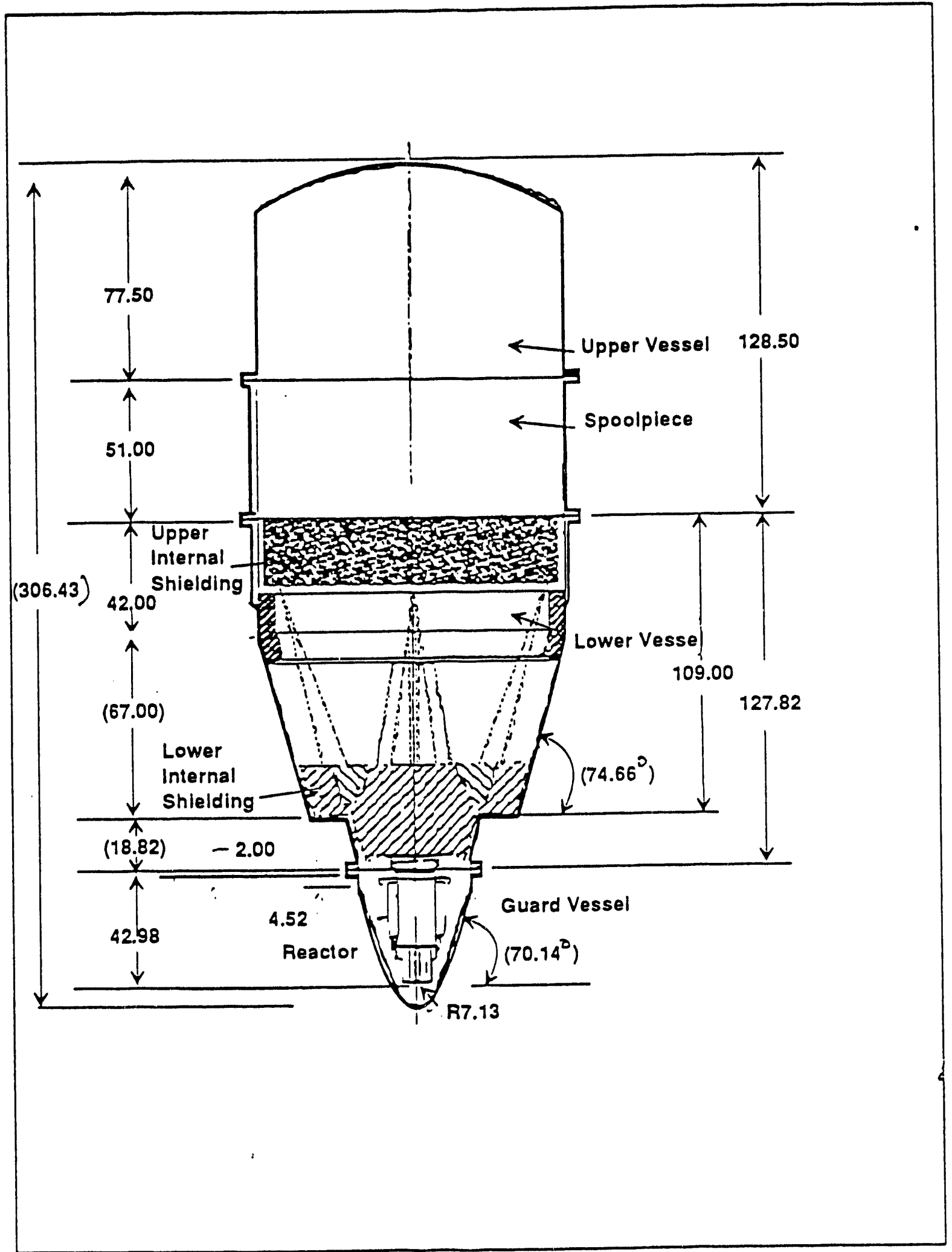

Figure 2. GES Test Site Vacuum Vessel 


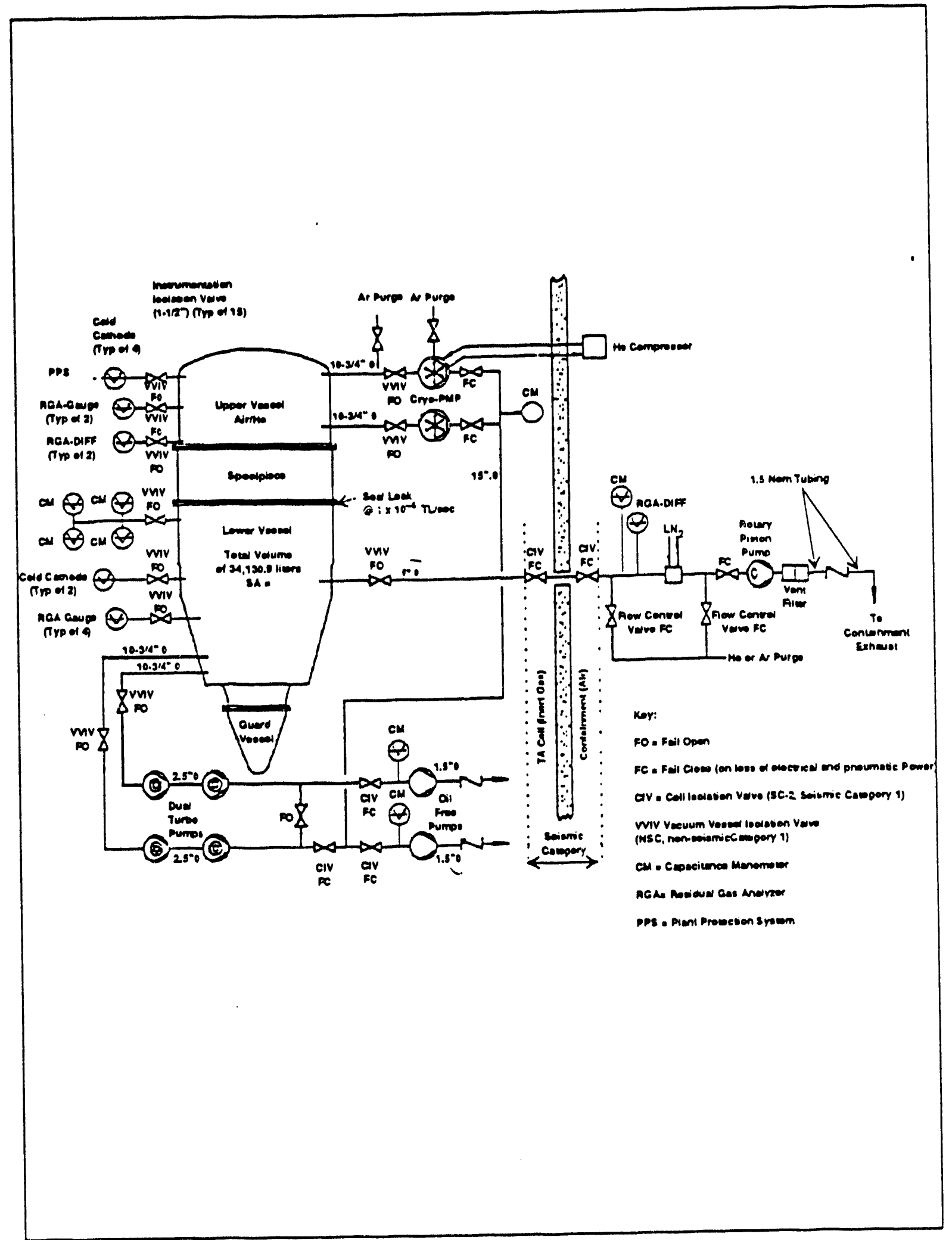

Figure 3. I GES Test Site Vacuum 


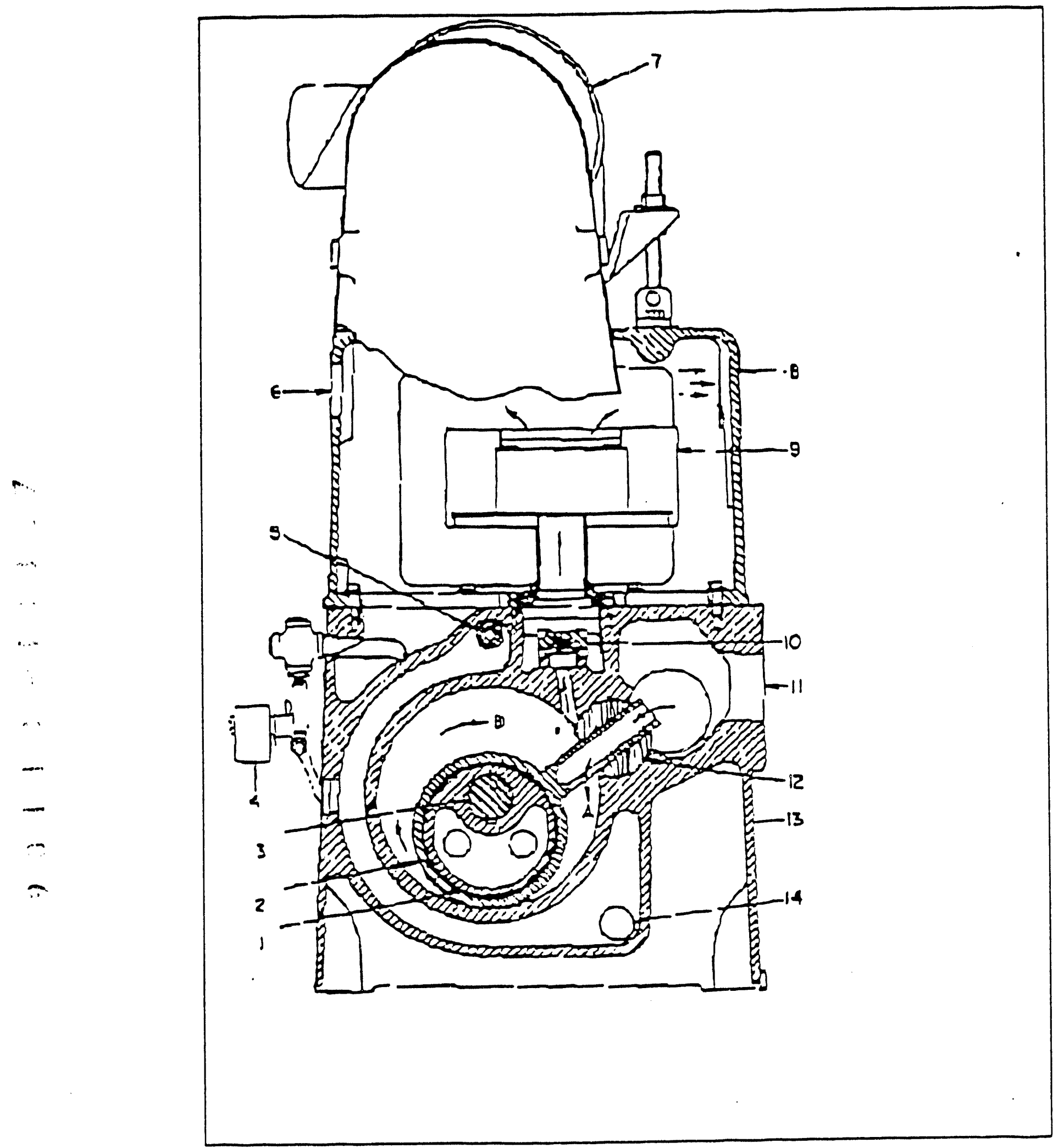

Figure 4. Roiary Fision Pump - 


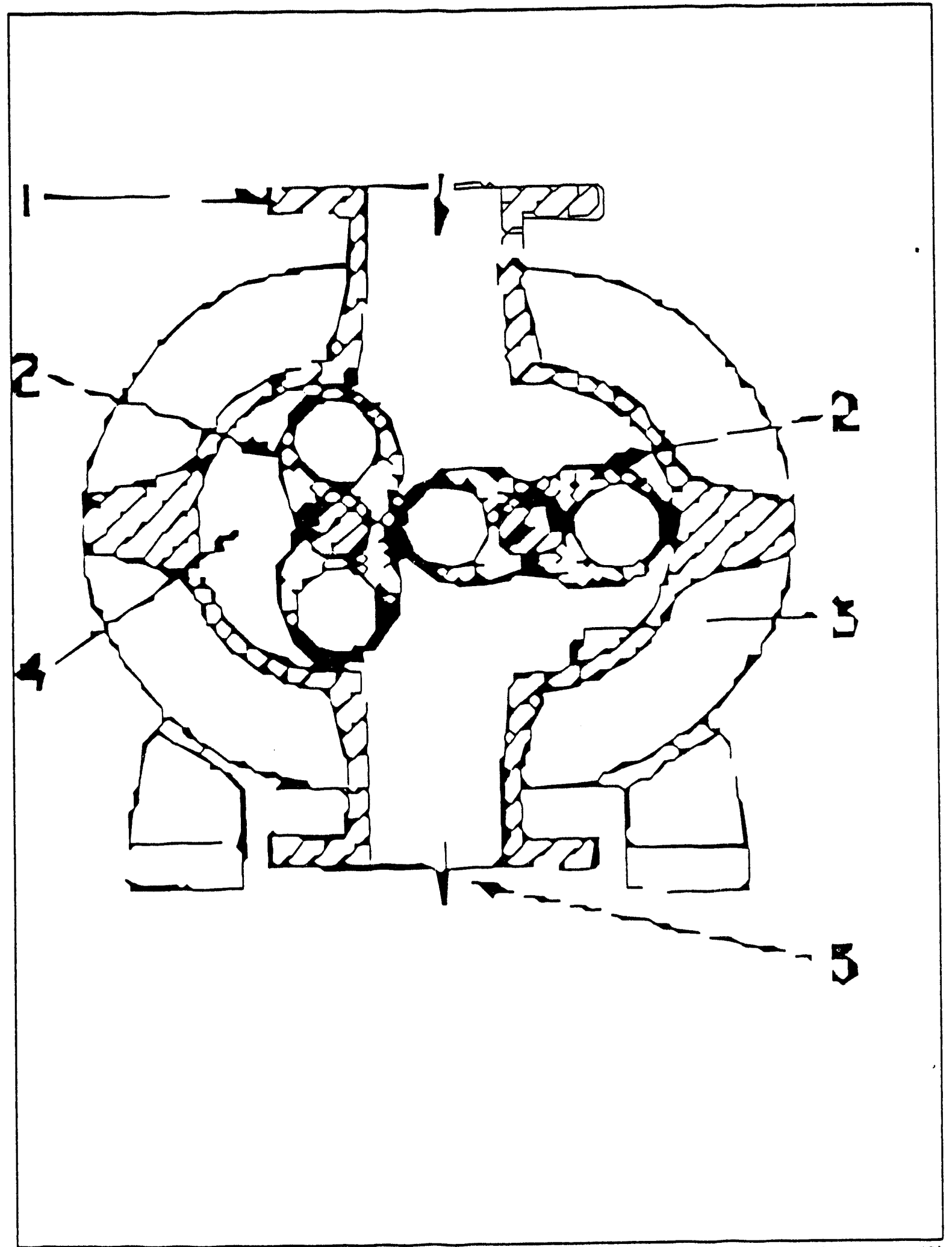

Figure 5. Roots Blower Pump; section through a single-stage roots pump; (1) inlet, (2) rotors, (3) housing, (4) Pump chamber (swept volume), (5) outlet. 
FIGURE 6

Turbamolecular Pump

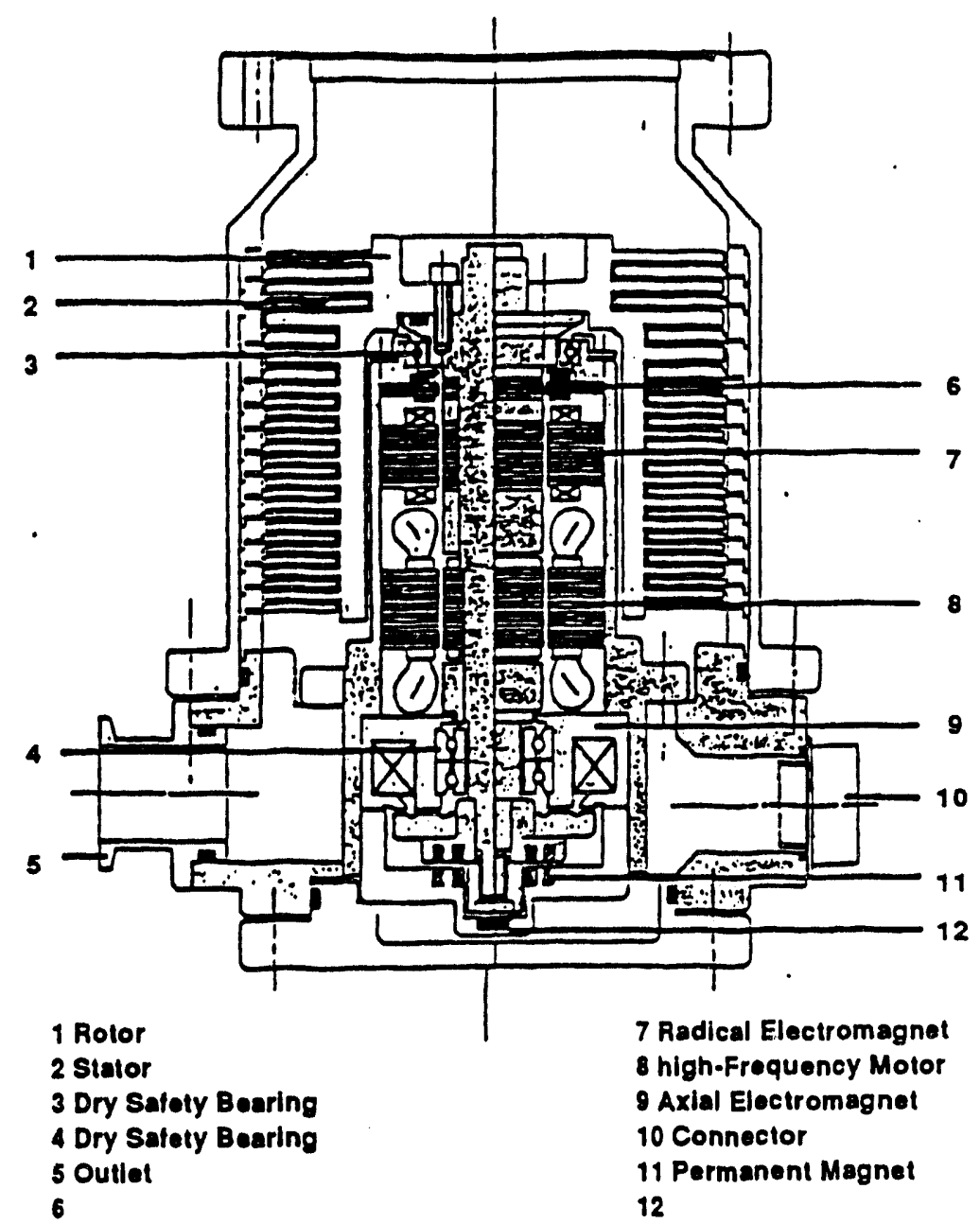


intersection of

butside Surface of Vessel

Centerline of Vecuum Roughing Line

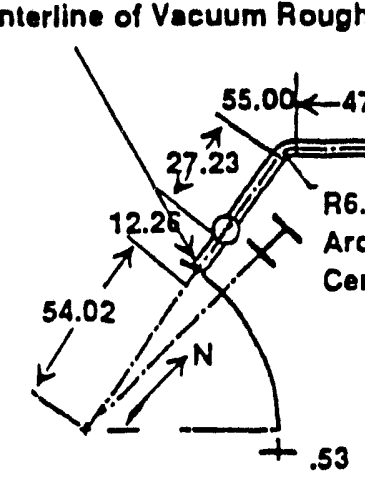

Plan View of Pump Gallex<smiles>CC(=[Te])C1(C)CCCCC1C</smiles>

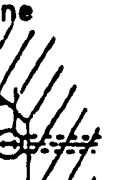

$1 /$
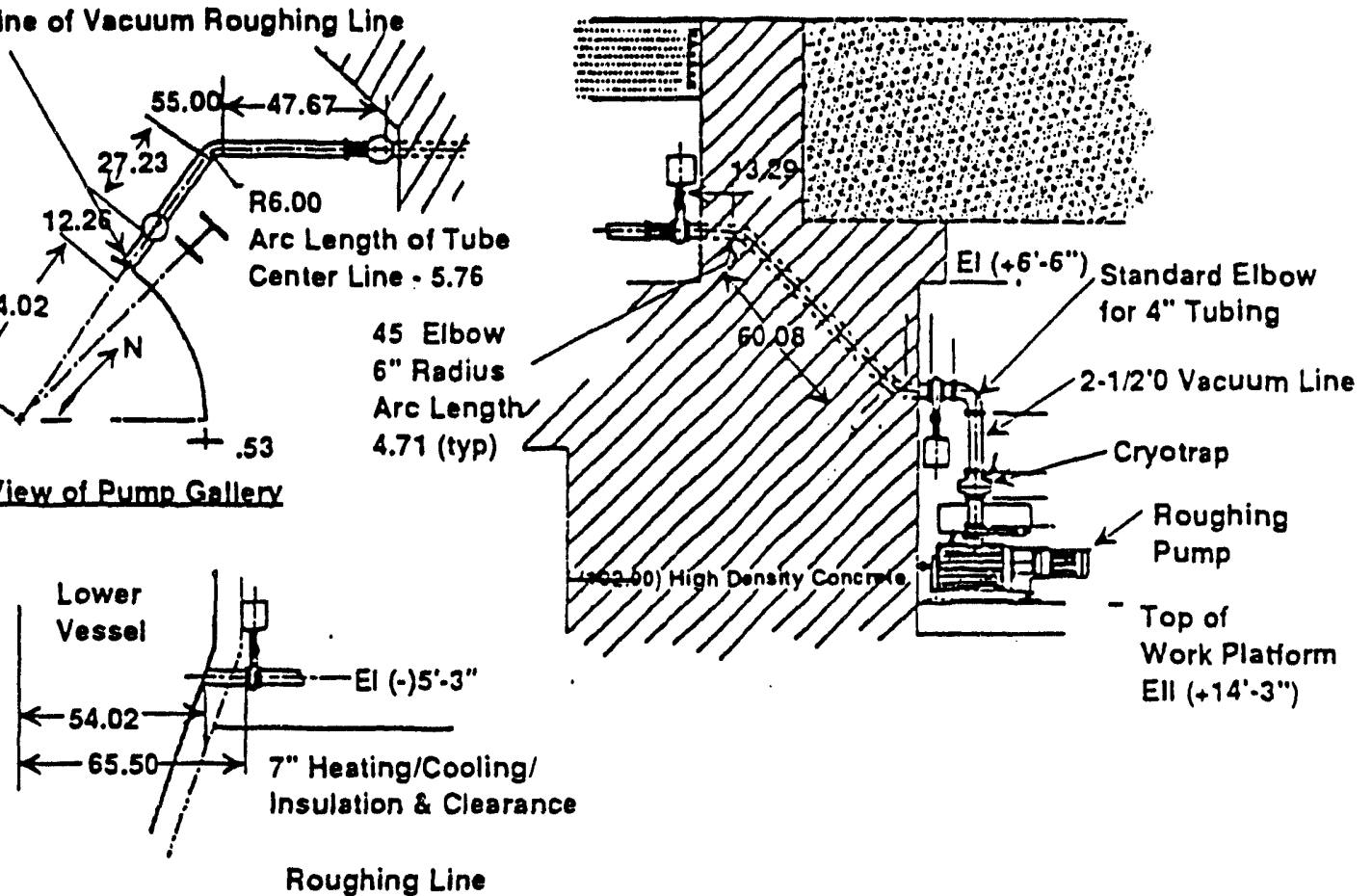

Elevation Line

Figure 7. Rough Vacurm System Configuration 
'dund Je|noejouroqun 6 asn6!y

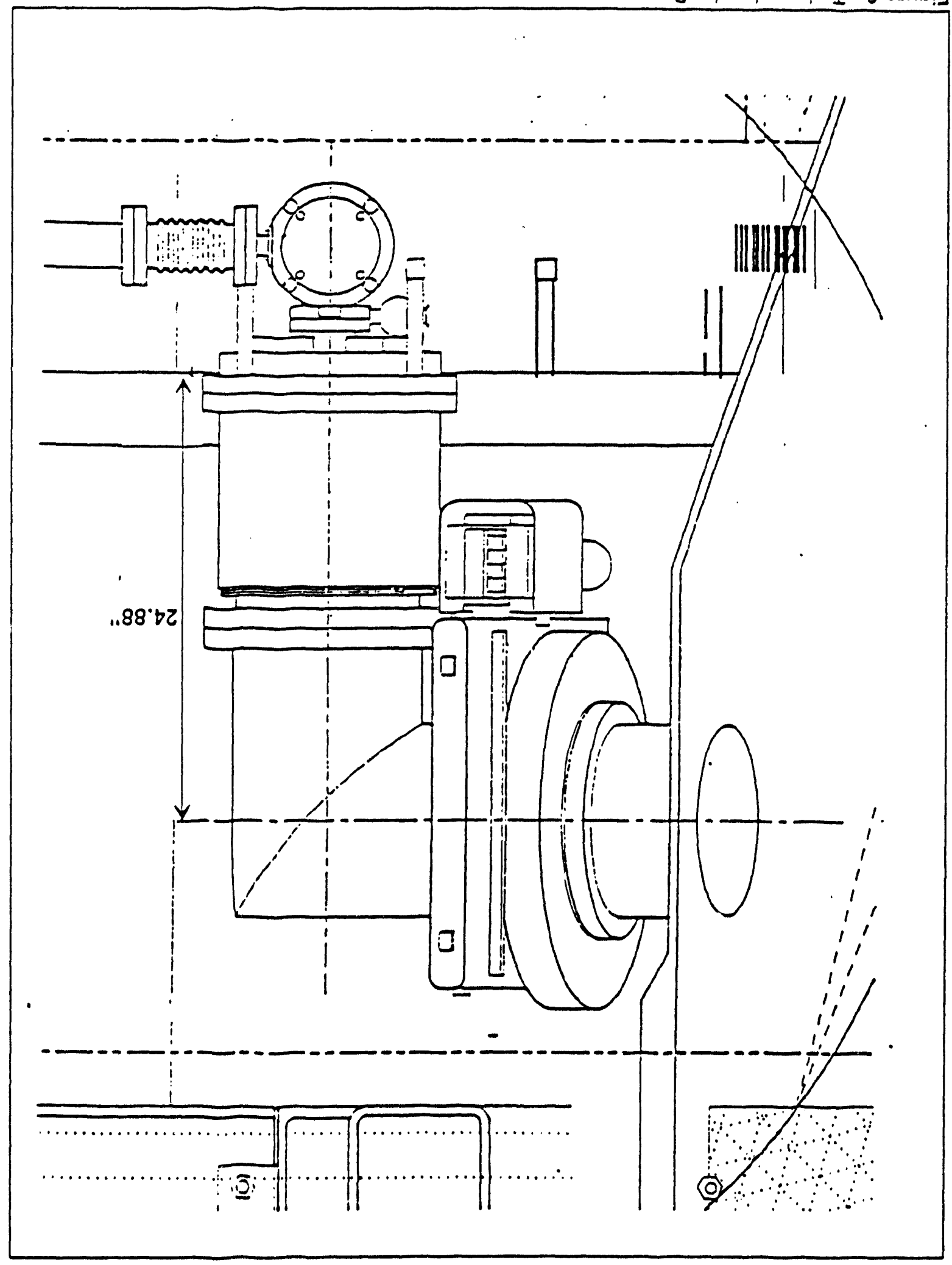




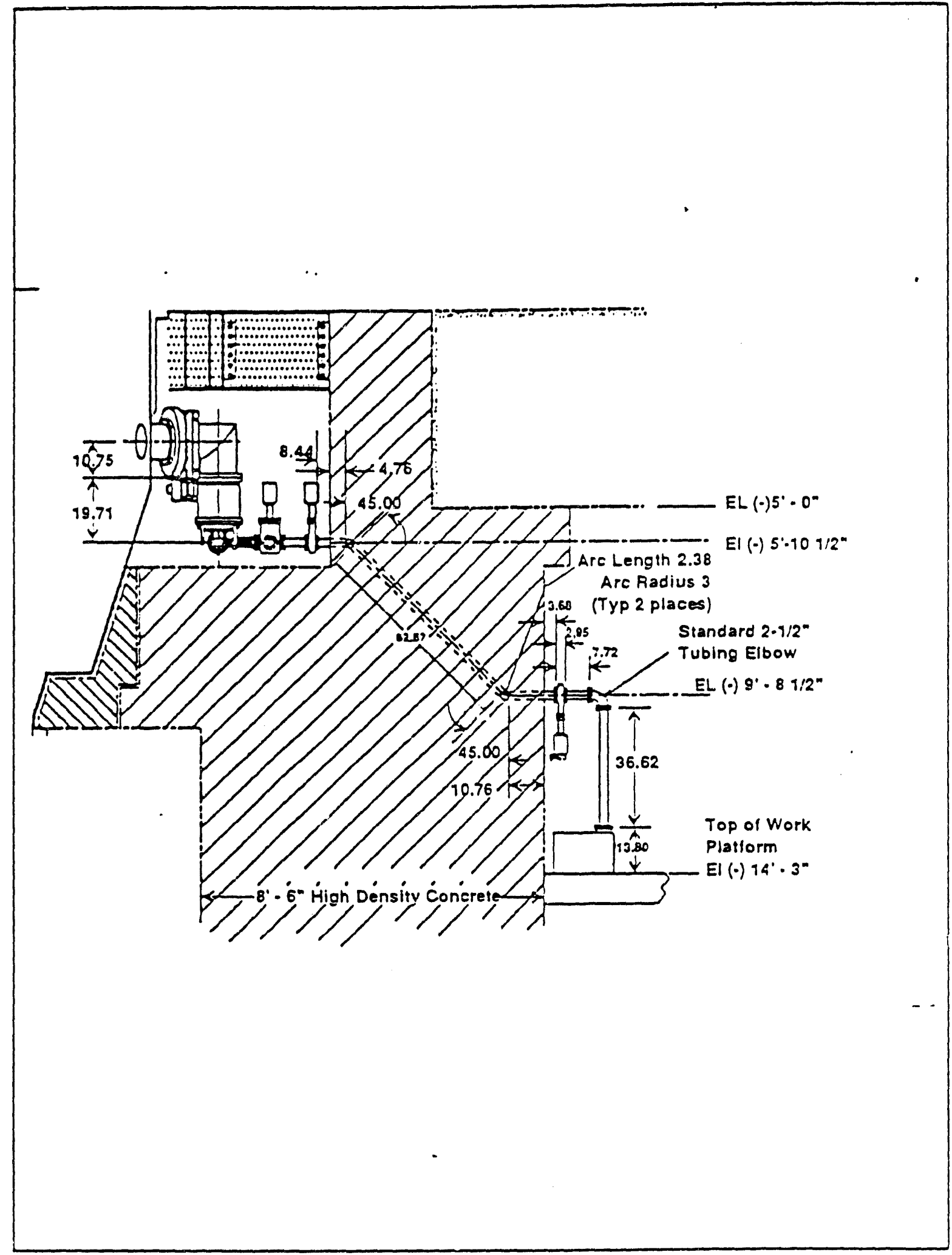

Figure 10. Turbopump elevation view. 


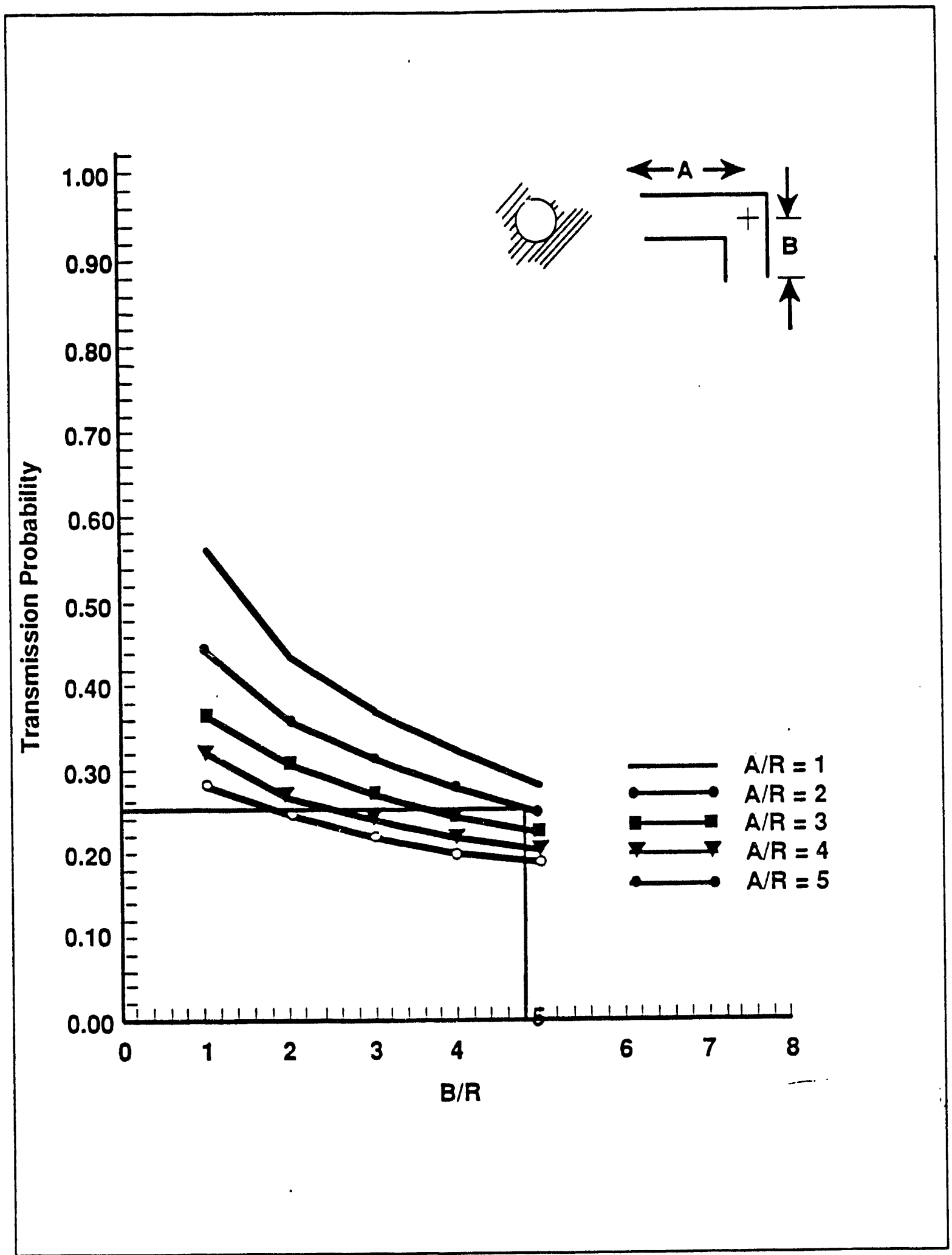

Figure 11. Transmission probabilities for elbow bend. 
FIGURE 12

Pump Curve - Air

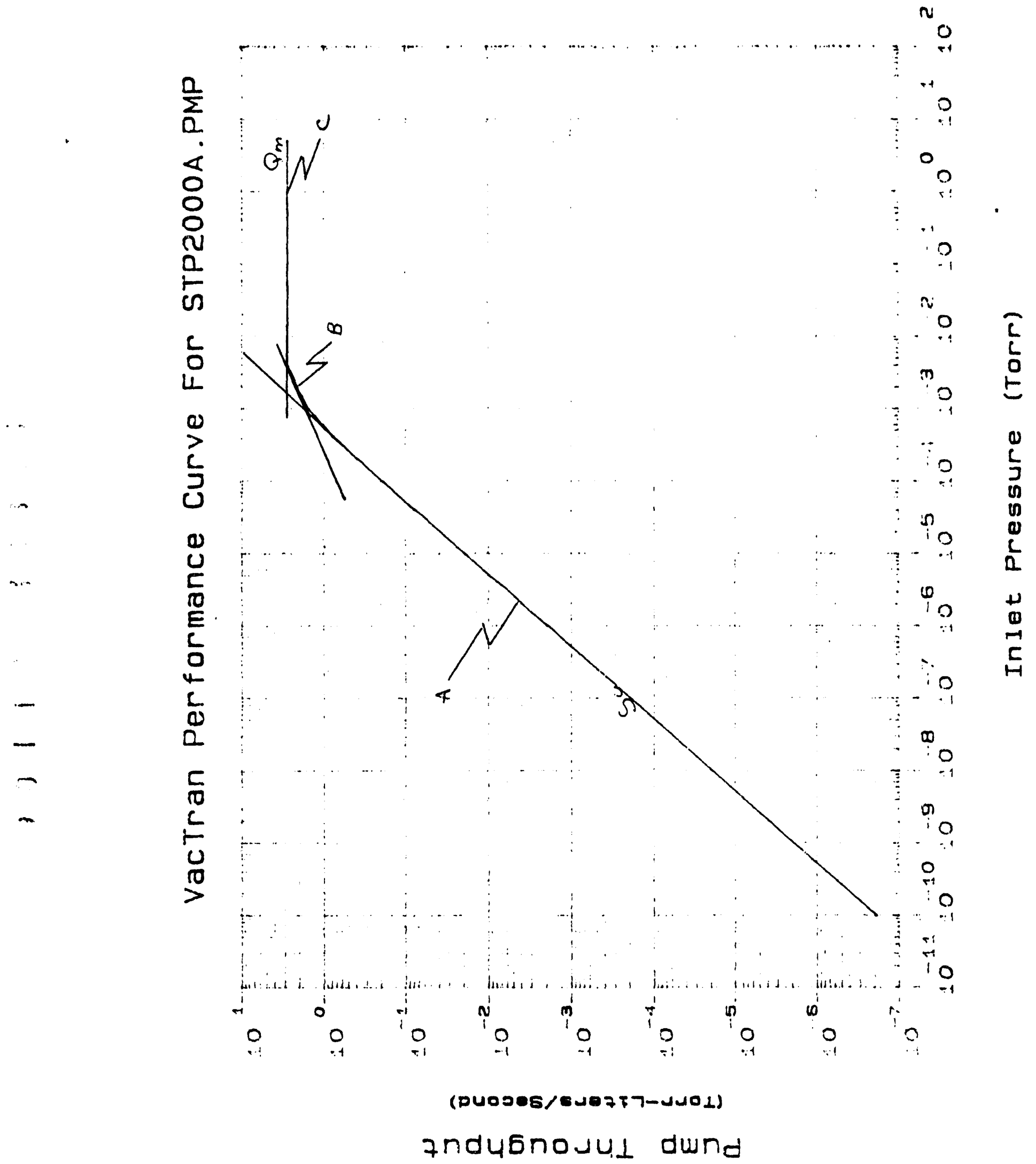


VacTran Performance Curve For STPHE.PMP

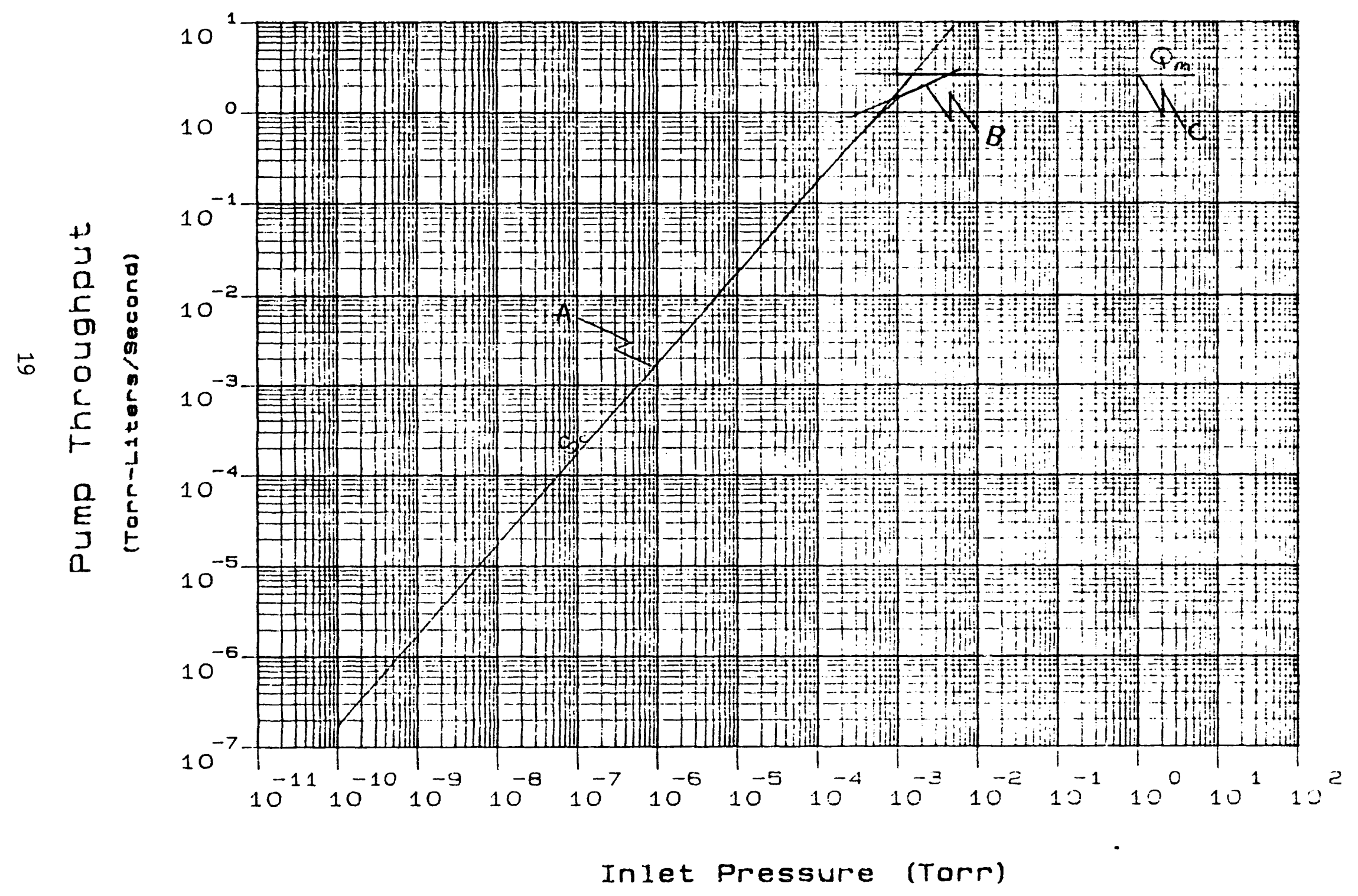


Vacuum System Analysis

Denise S Rohrdanz

July 19,1990

\section{Appendix A}

Several configurations were analyzed for both air and helium, with system descriptions defined below. The system consists of one roughing pump and one or two turbomolecular pumps attached to the vessel by ducting. The roughing pump, a rotary piston, or roots blower pump, is located at station. one, external to the Test Cell. The turbomolecular pumps are located at stations two and three as applicable, within the Test Cell. In all cases, gas load was assumed to be 1.1E-6 torr-liters per sec., air, for initial pump down (air in Test Cell) and helium for follow on pump downs (with an inerted Test (ell).

Case 1 : Air, 1 ea. DK200 \& 2 ea. STP2000

VacTran System Model: RUF.sys

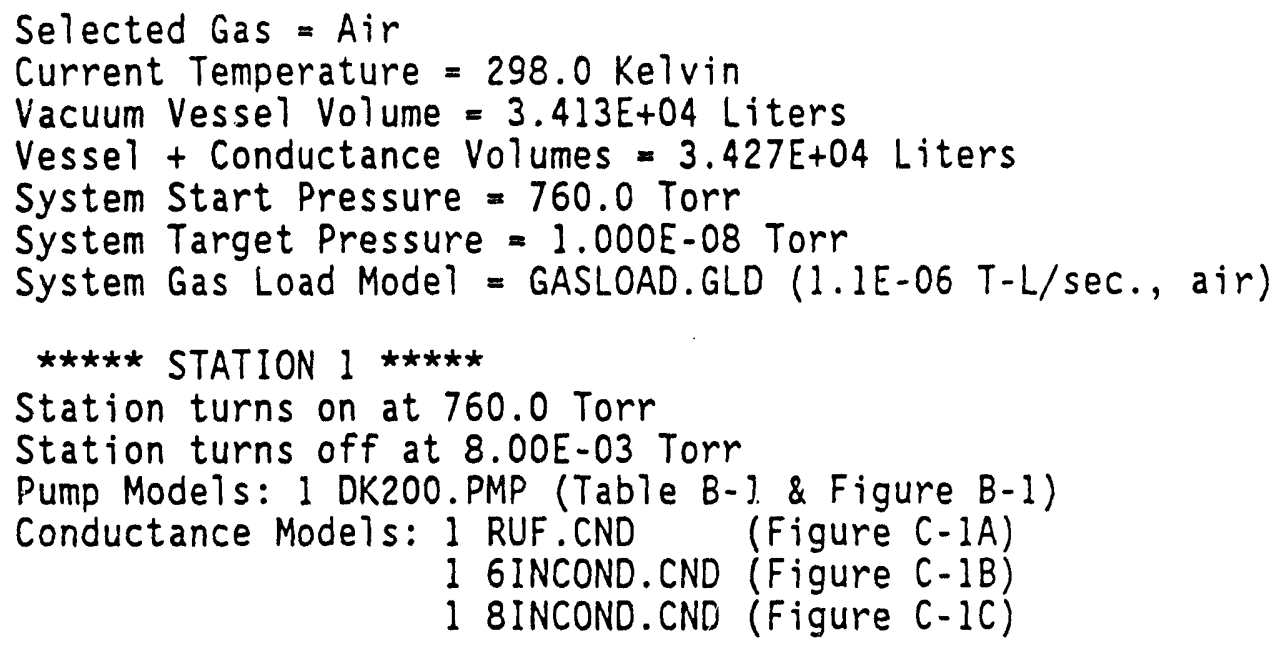

$\star \star \star \star * *$ STATION $2 * \star * * *$

Station turns on at 0.008 Torr

Station turns off at $1.000 E-08$ Torr

Pump Models: 1 STP2000A.PMP (Table B-3, Figure B-5)

Conductance Models: 1 TURBO.CND (2226 1/sec., air)

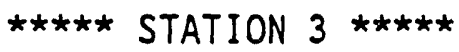

Station turns on at 0.008 Torr

Station turns off at 1.000 -08 Torr

Pump Models: 1 STP2000A.PMP (Table B-3, Figure B-5)

Conductance Models: 1 TURBO.CND (2226 i/sec., air)

Total Pumpdown Time: $2 \mathrm{hr}-40 \mathrm{~min}$ ( $4-i n$. duct)

$2 \mathrm{hr}-20 \min (6-i n$. duct)

$2 \mathrm{hr}-18 \mathrm{~min}(8-\mathrm{in}$. duct) 
Vacuum System Analysis

Denise S Rohrdanz

Juty 19, 1990

\section{Appendix A}

Case 2: Air, 1 ea. DU0250 \& 2 ea. STr2000

VacTran System Model RF2.SYS

Selected Gas = Air

Current Temperature $=298.0$ Kelvin

Vacuum Vessel Volume $=3.413 E+04$ Liters

Vesse $1+$ Conductance Volumes $=3.427 \mathrm{E}+04$ Liters

System Start Pressure $=760.0$ Torr

System Target Pressure $=1.000 E-08$ Torr

System Gas Load Model = GASLOAD.GLD (1.1E-06 T-L/sec., air)

$* \star \star * \star$ STATION 1

Station turns on at 760.0 Torr

Station turns off at 8.00E-03 Torr

Pump Models: 1 DU0250A.PMP (Table B-2, Figure B-5)

Conductance Models: 1 RUF.CND (Figure $C-1 A$ )

1 6INCOND.CND (Figure $C-1 B$ )

1 8INCOND.CND (Figure $C-I C$ )

$\star \star \star \star \star *$ STATION 2

Station turns on at 0.008 Torr

Station tirns off at $1.000 E-08$ Torr

Pump Models: 1 STP2000A. PMP (Table B-3, Figure B-5)

Conductance Models: 1 TURBO.CND (2226 1/sec., air)

$\star \star \star \star \star \star$ STATION $3 * \star \star \star \star \star *$

Station turns on at 0.008 Torr

Station turns off at $1.000 E-08$ Torr

Pump Models: 1 STP2000A.PMP (Table B-3, Figure B-5)

Conductance Models: 1 TURBO.CND (2226 1/sec., air)

Total Pumpdown Time: $2 \mathrm{hr}-05 \mathrm{~min}$ (4-in. duct)

$1 \mathrm{hr}-45 \mathrm{~min}(6-i n$. duct)

$1 \mathrm{hr}-41 \mathrm{~min}(8-i n$. duct) 
Appendix A

Case 3: Air, 1 ea. DK200 \& 1 ea. STP2000

VacTran System Mode1 RF3.SYS

Selected Gas = Air

Current Temperature $=298.0$ Kelvin

Vacuum Vessel Volume $=3.413 E+04$ Liters

Vessel + Conductance Volumes $=3.422 E+04$ Liters

System Start Pressure $=760.0$ Torr

System Target Pressure $=1.000 E-08$ Torr

System Gas Load Model = GASLOAD.GLD (1.1E-06 T-L/sec., air)

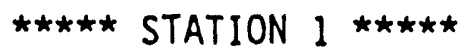

Station turns on at 760.0 Torr

Station turns off at 8.00E-03 Torr

Pump Models: 1 DK200.PMP (Table B-1, Figure B-1)

Conductance Models: 1 RUF.CND (Figure $C-1 A$ )

1 6INCOND.CND (Figure $C-1 B$ )

1 BINCOND.CND (Figure $C-1 C$ )

$\star * \star \star \star$ STATION 2

Station turns on at 0.008 Torr

Station turns off at $1.000^{\circ} 08$ Torr

Pump Models: 1 STP2000A.PMP (Table B-3, Figure B-5)

Conductance Models: 1 TURBO.CND (2226 1/sec., air)

Total Pumpdown Time: $2 \mathrm{hr}-45 \mathrm{~min}$ ( $4-\mathrm{in}$. duct)

$2 \mathrm{hr}-24 \min (6-i n$. durt)

$2 \mathrm{hr}-20 \mathrm{~min}(8-i n$. juct) 
Appendix A

Case 4: Air, 1 ea. Stokes Dry Pump 6210 \& 2 ea. STP2000

VacTran System Model STOKES.SYS

Selected Gas = Air

Current Temperature $=298.0$ Kelvin

Vacuum Vessel Volume $=3.000 E+04$ Liters

Vesse $1+$ Conductance Volumes $=3.014 E+04$ Liters

System Start Pressure $=760.0$ Torr

System Target Pressure $=1.000 \mathrm{E}-08$ Torr

System Gas Load Mode1 = GASLOAD.GLD

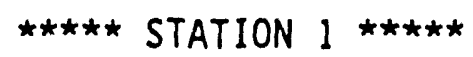

Station turns on at 760.0 Torr

Station turns off at 0.008 Torr

Pump Models: 1 STOKES. PMP (Table B-5, Figure B-8)

Conductance Models: 1 RUF. CND (Figure $C-1 A$ )

1 6INCOND.CND (Figure $C-1 B$ )

1 8INCOND.CND (Figure C-IC)

$\star \star \star \star \star *$ STATION $2 * \star \star \star \star *$

Station turns on at 0.008 Torr

Station turns off at 1.000 E-08 Torr

Pump Models: 1 STP2000A.PMP (Table B-3, Figure B-5)

Conductance Models: I TURBO.CND (2226 i/sec., air)

$\star \star \star \star * *$ STATION $3 * \star \star \star * *$

Station turns on at 0.008 Torr

Station turns off at 1.000 E-08 Torr

Pump Models: 1 STP2000A.PMP (Table B-3, Figure B-5)

Conductance Models: I TURBO.CND (2226 1/sec., air)

Total Pumpdown Time: $2 \mathrm{hr}-20 \mathrm{~min}$ ( $4-\mathrm{in}$. duct)

$2 \mathrm{hr}-03$ min $(6-i n$. duct)

$1 \mathrm{hr}-59 \mathrm{~min}(8-i n$. duct) 


\section{Appendix A}

Case 5: Helium, 1 ea. DK200 \& 2 ea. STP2000

VacTran System Model HE.SYS

Selected Gas - Helium

Current Temperature $=298.0$ Kelvin

Vacuum Vessel Volume $=3.413 E+04$ Liters

Vesse $1+$ Conductance Volumes $=3.427 E+04$ Liters

System Start Pressure $=760.0$ Torr

System Target Pressure $=1.000 E-08$ Torr

System Gas Load Model = GASLOAD.GLD (1.1E-06 T-L/sec., He)

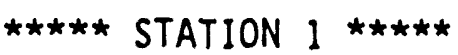

Station turns on at 760.0 Torr

Station turns off at 4.00E-03 Torr

Pump Models: 1 DK200.PMP (Table B-1, Figure B-1)

Conductance Models: 1 RUF.CND (Figure C-2A)

1 6INCOND.CND (Figure $C-2 B$ )

1 8INCOND.CND (Figure $C-2 C$ )

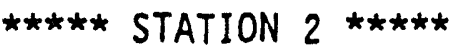

Station turns on at 0.004 Torr

Station turns off at $1.000 E-08$ Torr

Pump Models: 1 STPHE.PMP (Table B-4, Figure B-6)

Conductance Models: I TURBOHE.CND (5889 l/sec., helium)

$\star \star \star \star * *$ STATION $3 * \star \star \star * *$

Station turns on at 0.004 Torr

Station turns off at 1.000E-08 Torr

Pump Models: 1 STPHE.PMP (Table B-4, Figure B-6)

Conductance Models: I TURBOHE.CND (5889 1/sec., hel ium)

Total Pumpdown Time: $2 \mathrm{hr}-44 \min$ (4-in. duct)

$2 \mathrm{hr}-25 \mathrm{~min}(6-i n$. duct)

$2 \mathrm{hr}-22 \min (8-i n$. duct) 


\section{Vacuum System Analysis \\ Denise S Rohrdanz \\ July 19, 1990}

\section{Appendix A}

Case 6: Helium, 1 ea. DU0250 \& 2 ea. STP2000

VacTran System Model HE2.SYS

Selected Gas - Helium

Current Temperature $=298.0$ Kelvin

Vacuum Vessel Volume $=3.413 E+04$ Liters

Vessel + Conductance Volumes $=3.427 E+04$ Liters

System Start Pressure $=760.0$ Torr

System Target Pressure $=1.000 E-08$ Torr

System Gas Load Model = GASLOAD.GLD (1.1E-06 T-L/sec., He)

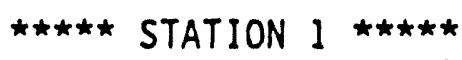

Station turns on at 760.0 Torr

Station turns off at 4.00E-03 Torr

Pump Mode1s: 1 DUO250A.PMP (Table B-2, Figure B-3)

Conductance Models: 1 RUF.CND (Figure $C-2 A$ )

1 6INCOND. CND (Figure $C-2 B$ )

1 SINCOND.CND (Figure $C-2 C$ )

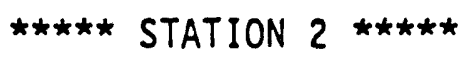

Station turns on at 0.004 Torr

Station turns off at 1.000E-08 Torr

Pump Models: 1 STPHE. PMP (Table B-4, Figure B-6)

Conductance Models: 1 TURBOHE.CND (5886 $\mathrm{l} / \mathrm{sec}$, helium)

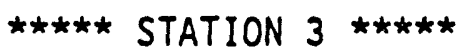

Station turns on at 0.004 Torr

Station turns off at 1.000E-08 Torr

Pump Models: 1 STPHE.PMP (Table B-4, Figure B-6)

Conductance Models: I TURBOHE.CND (5886 l/sec., helium)

Total Pumpdown Time: $2 \mathrm{hr}-08 \mathrm{~min}(4-\mathrm{in}$. duct)

$1 \mathrm{hr}-50 \mathrm{~min}(6-i n$. duct $)$

$1 \mathrm{hr}-46 \mathrm{~min}(8-i n$. duct) 
Appendix A

Case 7: Helium, 1 ea. DK200 \& 1 ea. STP2000

VacTran System Model HE4.SYS

Selected Gas = Helium

Current Temperature $=298.0 \mathrm{Kelvin}$

Vacuum Vessel Volume $=3.413 E+04$ Liters

Vesse $1+$ Conductance Volumes $=3.422 E+04$ Liters

System Start Pressure $=760.0$ Torr

System Target Pressure $=1.000$ E-08 Torr

System Gas Load Model - GASLOAD.GLD (1.1E-06 T-L/sec., He)

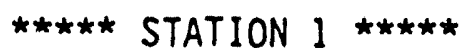

Station turns on at 760.0 Torr

Station turns off at $4.00 E-03$ Torr

Pump Models: 1 DK200. PMP (Table B-1, Figure B-1)

Conductance Models: I RUF.CND (Figure $C-2 A$ )

1 6INCOND. CND (Figure $C-2 B$ )

1 8INCOND. CND (Figure $C-2 C$ )

$\star \star \star \star * *$ STATION $2 * \star \star \star \star *$

Station turns on at 0.004 Torr

Station turns off at $1.000 E-08$ Torr

Pump Models: 1 STPHE. PMP (Table B-4, Figure B-6)

Conductance Models: I TURBOHE.CND ( $5886 \mathrm{l} / \mathrm{sec}$., helium)

Total Pumpdown Time: $2 \mathrm{hr}-46 \mathrm{~min}(4-i n$. duct)

$2 h r-28 \min (6-i n$. duct)

$2 \mathrm{hr}-25 \min (8-i n$. duct) 
Appendix A

Case 8: Helium, 1 ea. Stokes Dry Pump 6210 \& 2 ea. STP2000

VacTran System Mode1 STOKESHE.SYS

Selected Gas = Helium

Current Temperature $=298.0$ Kelvin

Vacuum Vessel Volume $=3.000 E+04$ Liters

Vesse $1+$ Conductance Volumes $=3.028 E+04$ Liters

System Start Pressure $=760.0$ Torr

System Target Pressure $=1.000 E-08$ Torr

System Gas Load Mode1 = GASLOAD.GLD

$\star \star \star \star \star \star \star$ STATION 1

Station turns on at 760.0 Torr

Station turns off at 0.008 Torr

Pump Models: 1 STOKES.PMP (Table B-5, Figure B-8)

Conductance Models: 1 RUF. CND (Figure $C-2 A$ )

1 6INCOND. CND (Figure $C-2 B$ )

1 BINCOND.CND (Figure $C-2 C$ )

$\star \star \star \star \star *$ STATION 2

Station turns on at 0.008 Torr

Station turns off at 1.000E-08 Torr

Pump Models: 1 STPHE. PMP (Table B-4, Figure B-6)

Conductance Models: 1 TURBOHE.CND (5886 $\mathrm{l} / \mathrm{sec}$., helium)

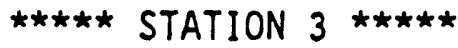

Station turns on at 0.008 Torr

Station turns off at 1.000E-08 Torr

Pump Models: 1 STPHE. PMP (Table B-4, Figure B-6)

Conductance Models: I TURBOHE.CND (5886 $\mathrm{l} / \mathrm{sec}$, helium)

Total Pumpdown Time: $2 \mathrm{hr}-14 \mathrm{~min}(4-\mathrm{in}$. duct)

$2 \mathrm{hr}$ (6-in. duct)

$1 \mathrm{hr}-58 \mathrm{~min}(8-\mathrm{in}$. duct) 


\section{Appendix B}

Pumps were compared on the bases of pump speed vs inlet pressure as shown below. In the case of the rotary piston and roots blower pumps, the same pump curve was used for both air and helium. Separate curves were plotted for the turbomolecular pumps.

\section{ROTARY PISTON PUMPS}

Pump: Leybold DK200

VacTran Pump Model DK200.PMP (Table B-1, Figure B-1)

Manufacturer's graph (Figure B-2)

Maximum Pump Speed $=115.2 \mathrm{Cu}$ Feet/Minute

Maximum Pump Pressure $=760.0$ Torr

Minimum Pump Pressure $=1.000$-05 Torr

Pump: Balzers DUO250A

VacTran Pump Model DU0250A.PMP (Table B-2, Figure B-3)

Manufacturer's graph (figure $B-4$ )

Maximum Pump Speed $=276.9$ Cu Meters/Hour

Maximum Pump Pressure $=760.0$ Torr

Minimum Pump Pressure $=2.000$ E-04 Torr

\section{TURBOMOLECULAR PUMP}

AIR:

Pump: Seiko STP2000

VacTran Pump Model STP2000A.PMP (Table B-3, Figure B-5)

Manufacturer's graph (Figure B-7)

Maximum Pump Speed $=1920$ Liters $/$ Second

Maximum Pump Pressure $=0.01$ Torr

Minimum Pump Pressure $=1.000 \mathrm{E}-10$ Torr

HELIUM:

Pump: Seiko STP2000

VacTran Pump Model STPHE.PMP (Table B-4, Figure B-6)

Manufacturer's graph (Figure B-7)

Maximum Pump Speed $=1780$ Liters $/$ Second

Maximum Pump Pressure $=0.006$ Torr

Minimum Pump Pressure $=1.000$ E-10 Torr 
Vacuum System Analysis Denise S Rohrdanz July 19, 1990

\section{ROOTS BLOWER PUMP}

Pump: Stokes Dry Pump ' 6210

VacTran Pump Model STOKES.PMP (Table B-5, Figure B-8)

Manufacturer's graph (Figure B-9)

Maximum Pump Speed $=56.63$ Liters $/$ Second

Maximum Pump Pressure $=760.0$ Torr

Minimum Pump Pressure $=0.001$ Torr 


\section{Vacuum System Analysis Denise $S$ Rohrdanz July 19, 1990}

\section{Appendix B}

TABLE B-1

Pump Speed vs Inlet Pressure for Leybold DK200

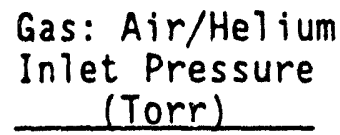

$7.60 E+02$

$5.00 E+02$

$2.00 E+00$

$1.00 E+02$

$1.00 E+01$

$1.00 E+00$

$1.00 E-00$

8.00E-02

$6.00 E-02$

$4.00 E-02$

$2.00 E-02$

$1.00 E-02$

$8.00 E-03$

$6.00 E-03$

$4.00 E-03$

$2.00 E-03$

1. $00 \mathrm{E}-03$

$8.00 E-04$

$6.00 E-04$

$4.00 E-04$

$2.00 E-04$

$1.00 E-04$

$8.00 E-05$

$6.00 E-05$

$4.00 E-05$

$2.00 E-05$

1. OOE-05
Speed

(Cu Feet/Minute)

1. $15 E+02$

1. $14 \mathrm{E}+02$

1. $14 E+02$

1. $13 E+02$

1. $11 E+02$

$1.08 E+02$

$1.02 E+02$

$1.02 E+02$

$1.01 E+02$

$9.99 E+01$

$9.70 E+01$

$9.36 E+01$

$9.20 E+01$

9. $10 E+01$

$8.80 E+01$

8. $30 E+01$

$7.67 E+01$

7. $40 E+01$

7. $10 E+01$

$6.60 E+01$

5.70E+01

$4.48 E+01$

4.10E+01

3. $50 E+01$

2. $50 E+01$

$7.00 E+00$

$1.00 E-01$
TABLE $B-2$

Pump Speed vs Inlet Pressure for Balzers DU0250A

Gas: Air/Helium

Inlet Pressure (Torr)

Speed

(Cu Meters/Minute)

$7.60 E+02$

$2.76 E+02$

$5.00 E+02$

$2.00 E+02$

$1.00 E+02$

$1.00 E+01$

$1.00 E+00$

$1.00 E-01$

8.00E-02

$6.00 E-02$

4. 00 E-02

$2.00 E-02$

1. 00 E- 02

$8.00 E-03$

$6.00 E-03$

$4.00 E-03$

2. $00 \mathrm{E}-03$

1. 00 E-03

8. 00 - 04

$6.00 E-04$

$4.00 E-04$

2. 00 E- 04
$2.76 \mathrm{E}+02$

$1.75 E+02$

2. $73 E+02$

$2.68 E+02$

2. $56 E+02$

2. $34 E+02$

2. $32 E+02$

2. $29 E+02$

2. $22 E+02$

2. $10 E+02$

$1.97 E+02$

1. $91 E+02$

$1.85 E+02$

$1.73 E+02$

1. $52 E+02$

1. $27 E+02$

1. $17 E+02$

1. $05 E+02$

$8.49 E+01$

$4.58 \mathrm{E}+01$ 
Vacuum System Analysis

Denise S Rohrdanz

July 19, 1990

Appendix B

TABLE B-3

Pump Speed vs Inlet Pressure for STP-2000

Gas: Air

Inlet Pressure (Torr)

$1.00 E-02$

$8.00 E-03$

$6.00 E-03$

$4.00 E-03$

$2.00 \mathrm{E}-03$

1. $00 \mathrm{E}-03$

$8.00 E-04$

$6.00 E-04$

4. $00 E-04$

$2.00 E-04$

1. $.00 E-04$

4. $00 \mathrm{E}-05$

$4.00 E-06$

$1.00 E-06$

1. $00 E-10$
Speed

(Liters/Second)

1.00E-01

$9.40 E+01$

$4.38 E+02$

$6.88 E+02$

1. $12 E+03$

$1.54 E+03$

$1.65 E+03$

$1.73 E+03$

$1.83 E+03$

$1.92 E+03$

1. $92 E+03$

1. $92 E+03$

1. $92 E+03$

1. $92 E+03$

1. $92 E+03$
TABLE B-4

Pump Speed vs Inlet Pressure for STP -2000

Gas: Helium

Inlet Pressure (Torr)

$6.00 E-03$

$4.00 E-03$

$2.00 E-03$

$1.00 E-03$

$8.00 E-04$

$6.00 E-04$

$4.00 E-04$

$2.00 E-04$

$1.00 E-04$

$4.00 E-05$

4. $.00 E-06$

$1.00 E-06$

$1.00 E-10$
Speed

(Liter/Second)

$1.00 E-01$

$3.13 E+02$

$7.82 E+02$

$1.25 E+03$

$1.38 \mathrm{E}+03$

$1.53 E+03$

$1.64 E+03$

$1.76 E+03$

$1.78 E+03$

$1.78 E+03$

$1.78 E+03$

$1.78 E+03$

$1.78 E+03$

TABLE B-5

Pump vs Inlet Pressure for Stokes Dry Pump 6210

Gas: Air/Helium

Inlet Pressure (Torr)

Speed

(Liters/Second)

7. $60 E+02$

$3.00 E+01$

2. $00 E+01$

$1.00 E+01$

$4.00 E-01$

2. $00 E-01$

$1.00 E-01$

$6.00 E-02$

4. OOE-02

$3.00 E-02$

2.00E-02

1. OOE-02

1. $00 \mathrm{E}-03$
$5.56 E+01$

$5.56 E+01$

$5.61 E+01$

5. $66 \mathrm{E}+01$

5.61E+01

$5.52 E+01$

5. $38 E+01$

5. $19 E+01$

$5.04 E+01$

4. $86 E+01$

$3.96 E+01$

2. $54 E+01$

4.71E+00 


\section{¿६ \\ Pump Speed \\ (Cu Foet/Minute)}

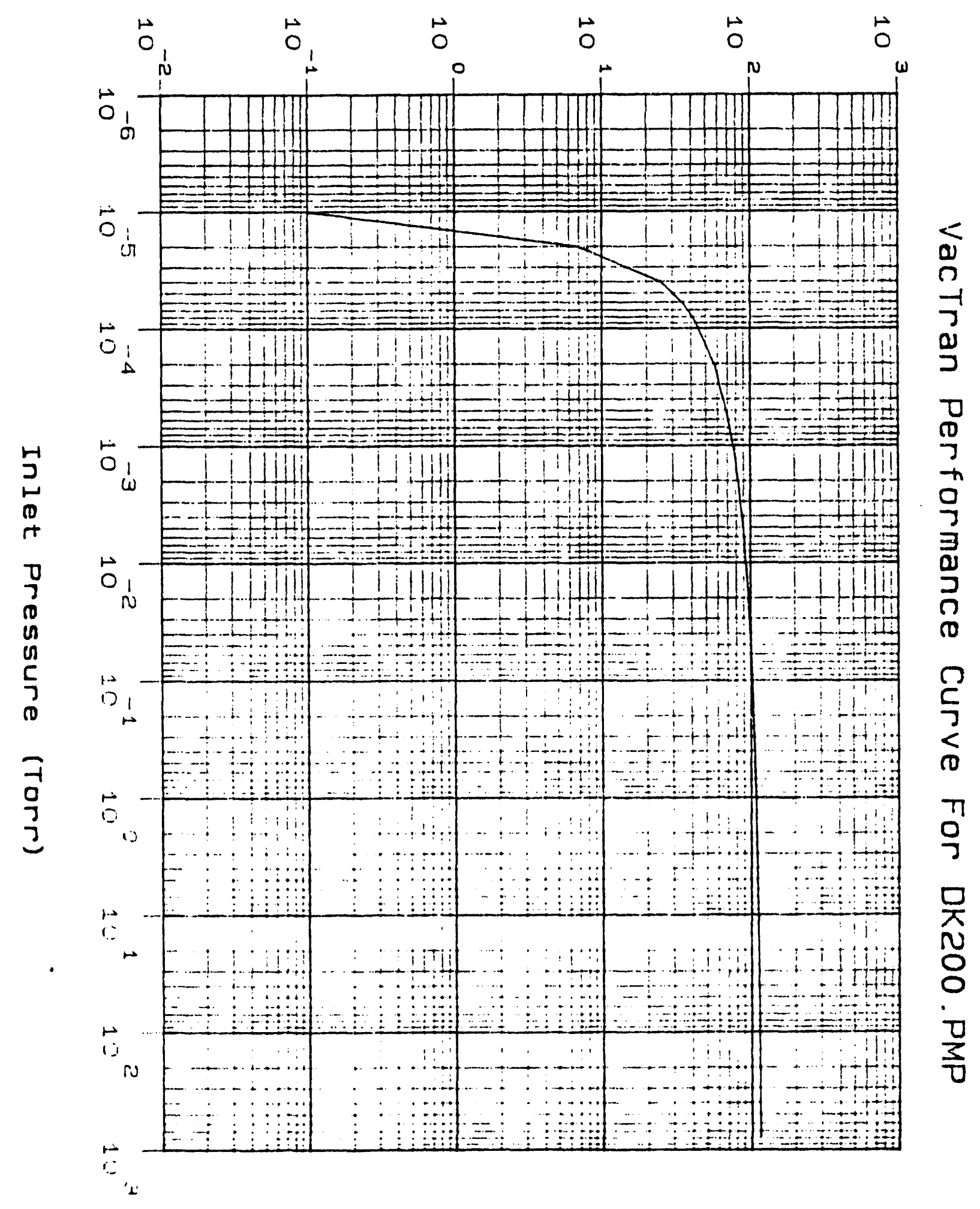

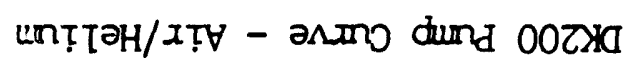

I-q J\&กDIA 
FIGURE B-2

DK200 MANUFACTURERS PUMP CURVE - AIR/HELIUM

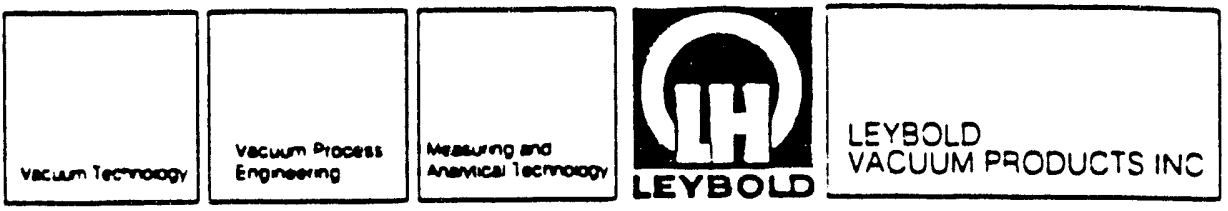

\section{Pertormance Curves}

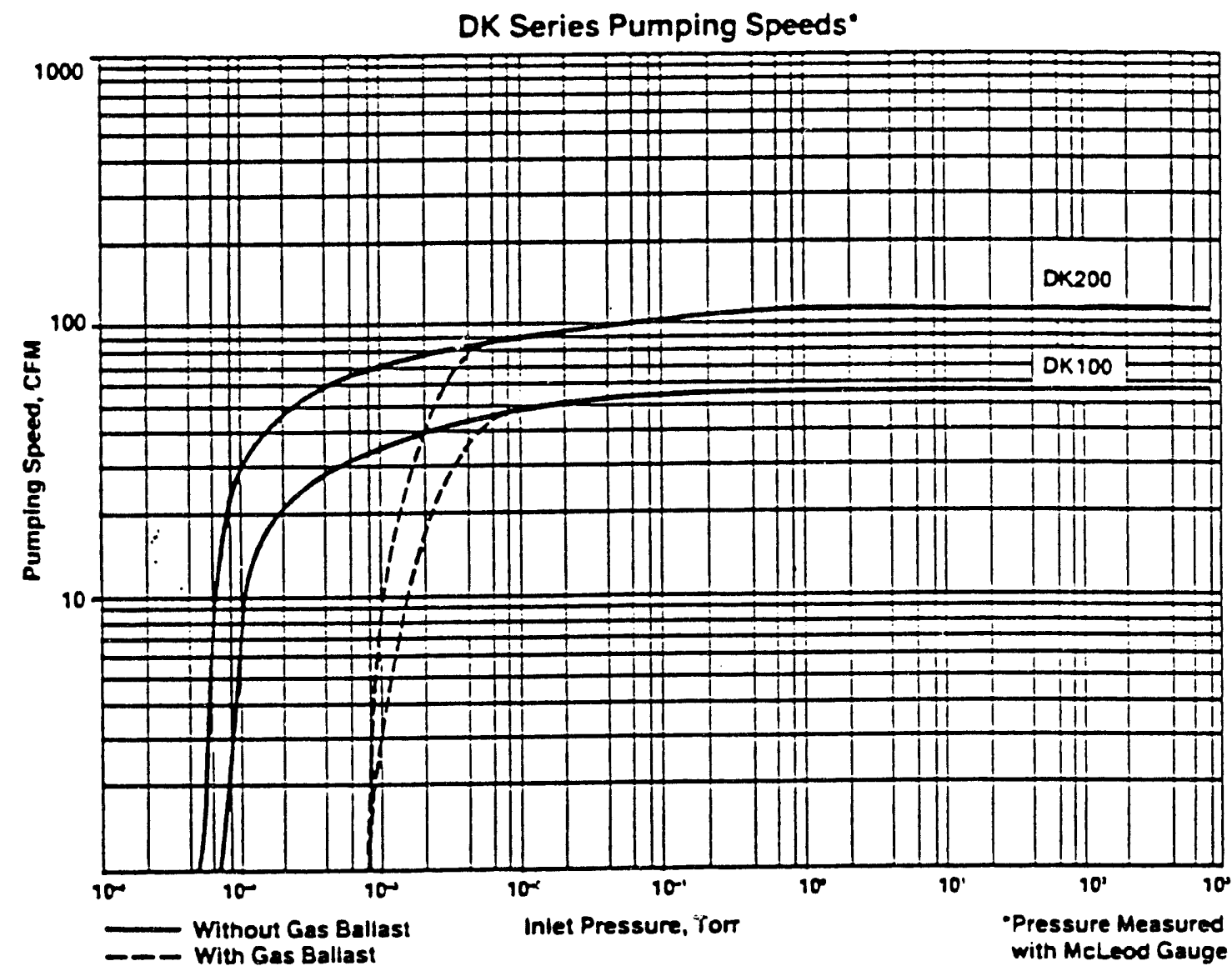




\section{DU0250A Pump Curve - Air/Helium}

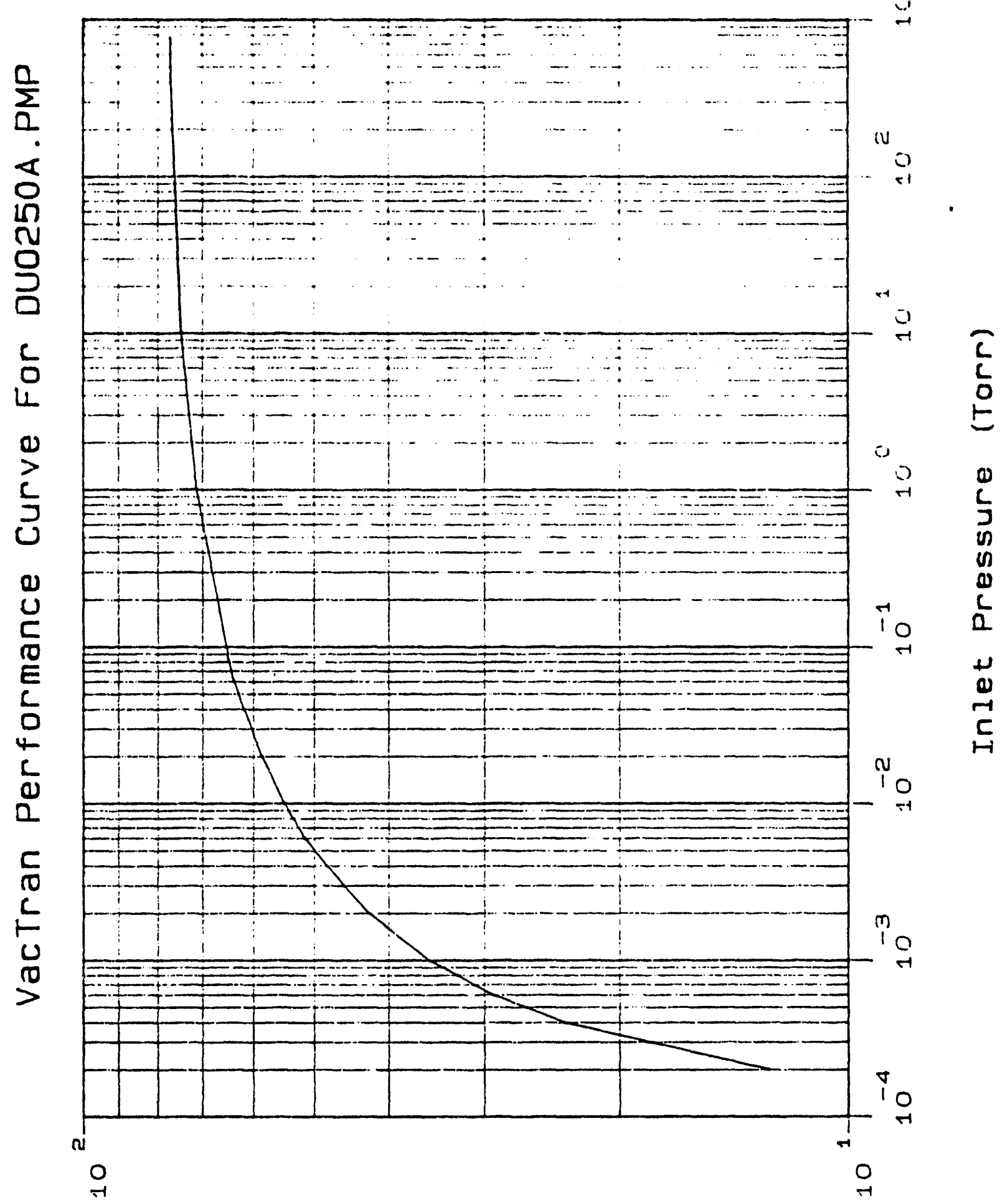

(Dบ0508/0407โ7)

paads dund 
FIGURE B-4

DUO250 MANUFACTURERS PUMP CURVE - AIR/HELIUM

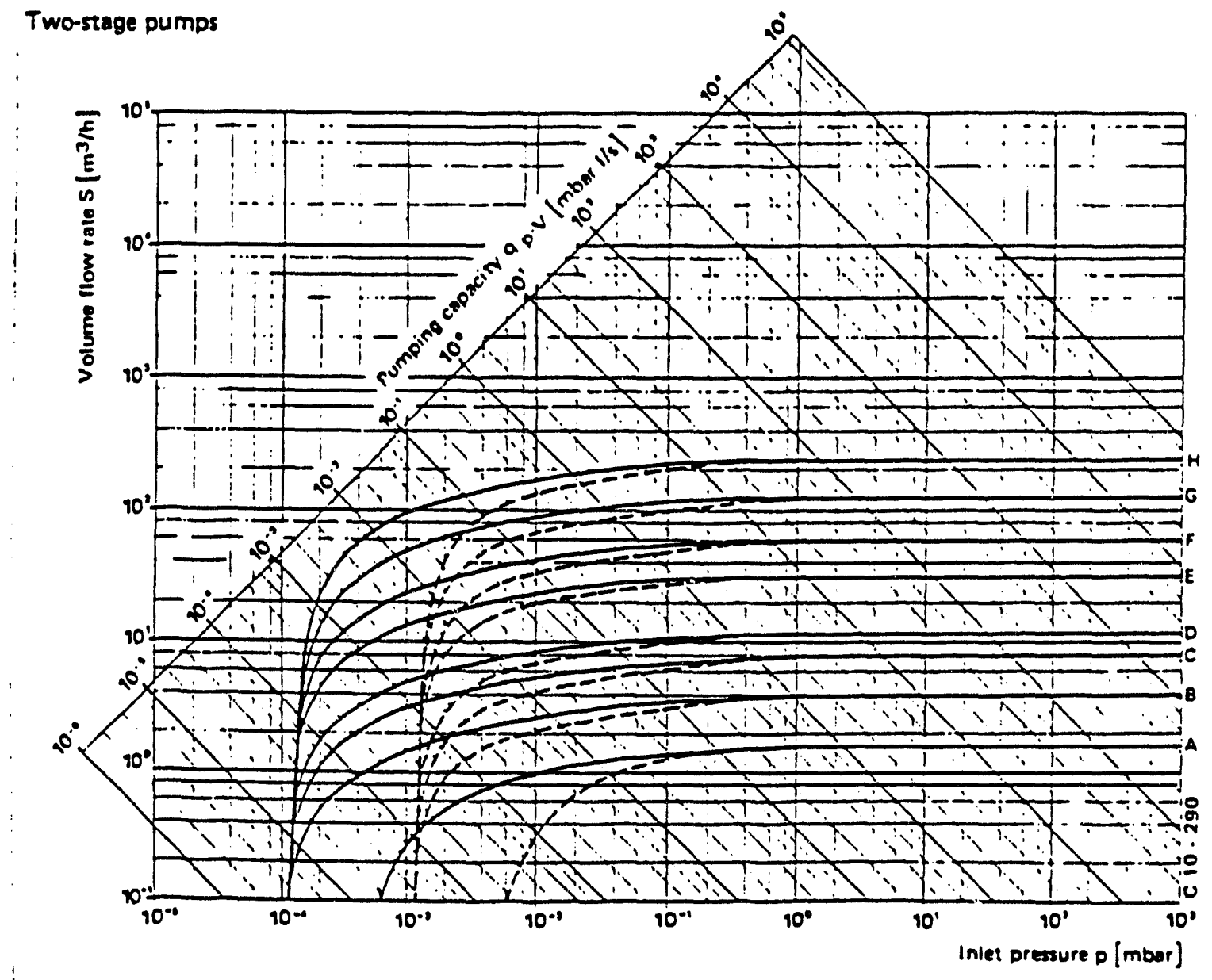

$A$ - DUO $1.5 \mathrm{~A}$

$B$ - DUO OOA A

C. DUO $008 \mathrm{~A}$

D. DUO012A

E DUO 030 A

F DUO 060 A

G - DUO $120 \mathrm{~A}$

H - DUO $250 \mathrm{~A}$ 
FIGURE B-5

STP2000 Pump Curve - Air

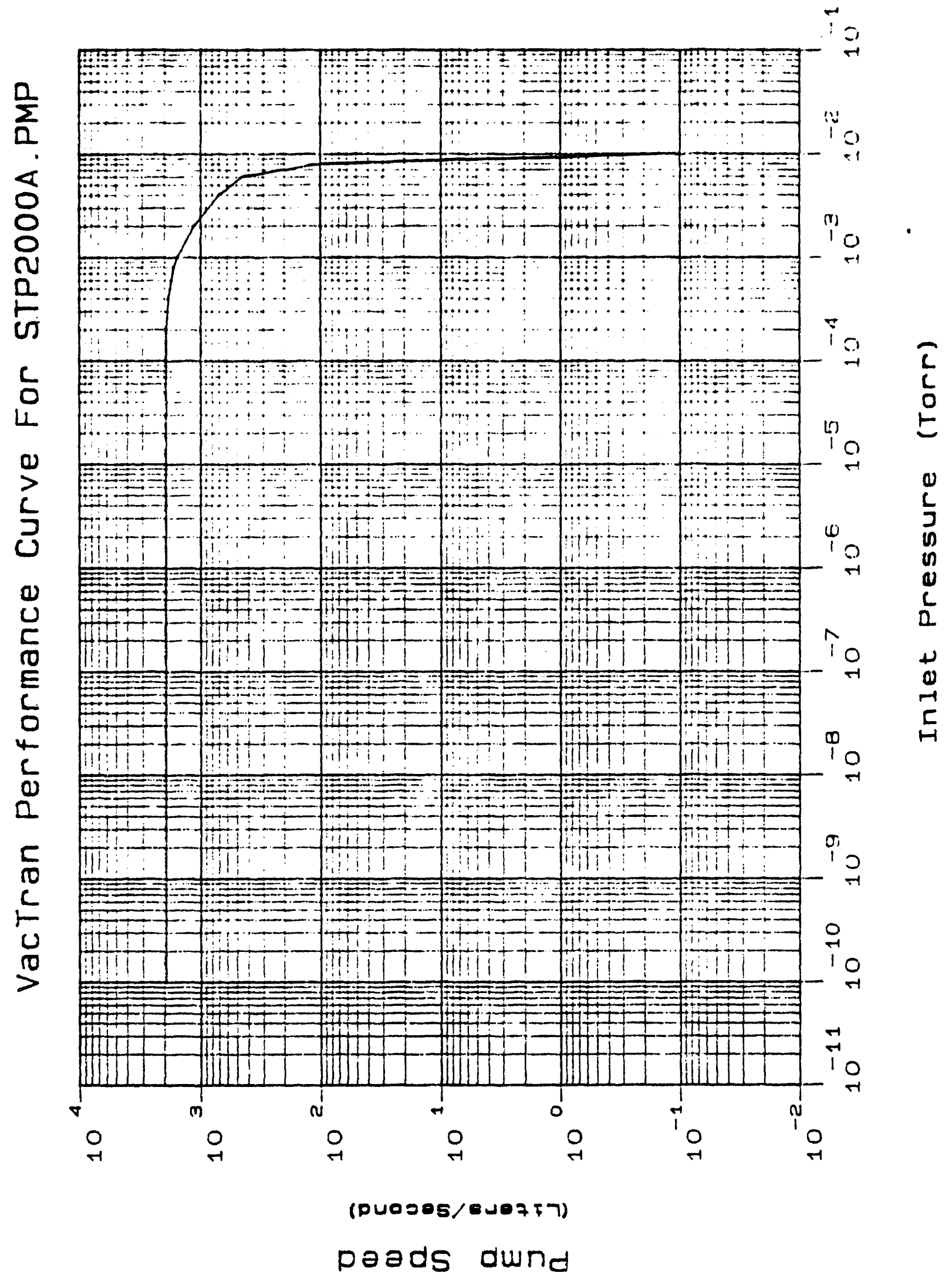


FIGURE B-6

STP2000 Pump Curve - Helium

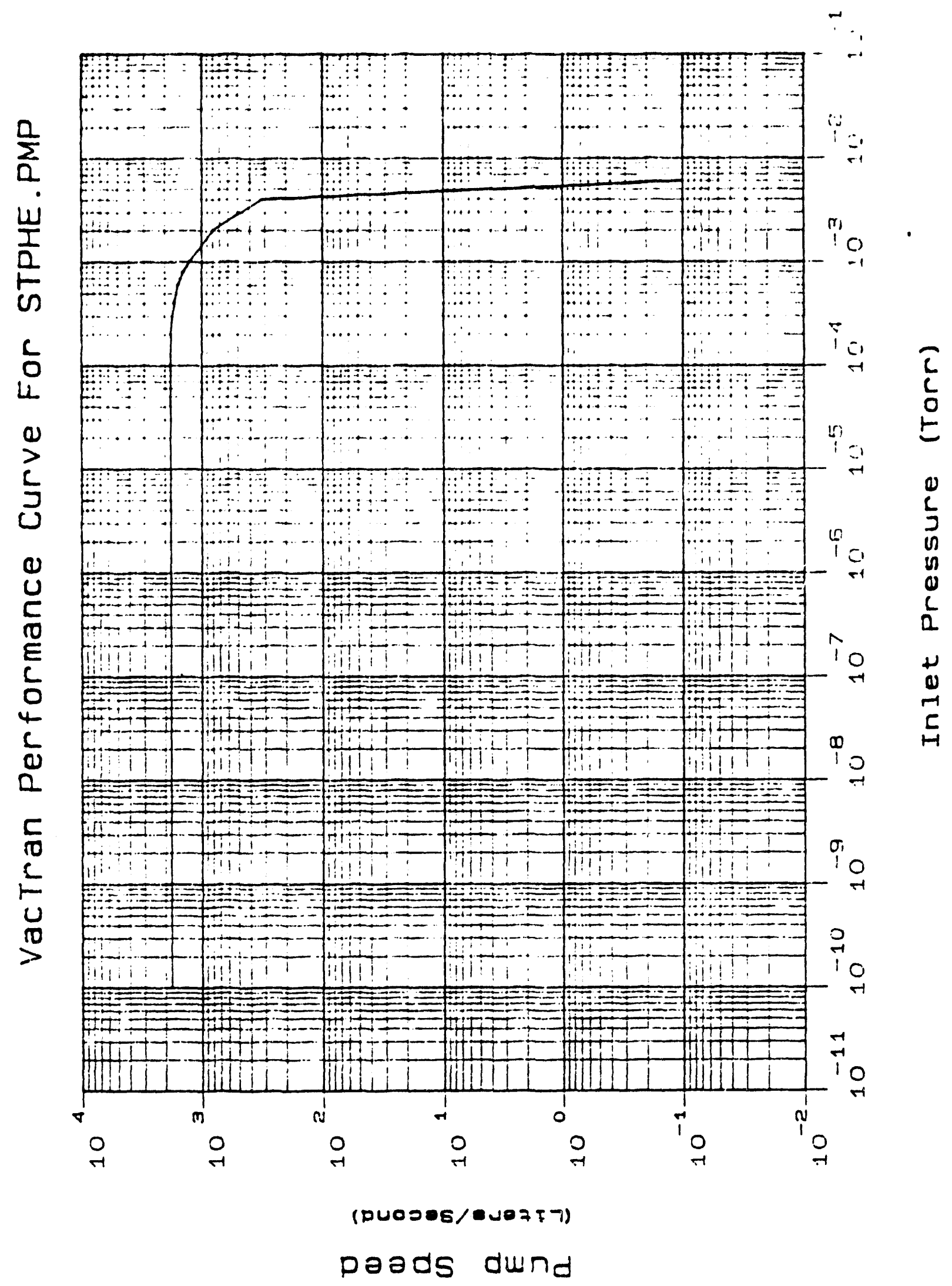


Pump Speed

\section{(Cu Feot/Minuta)}

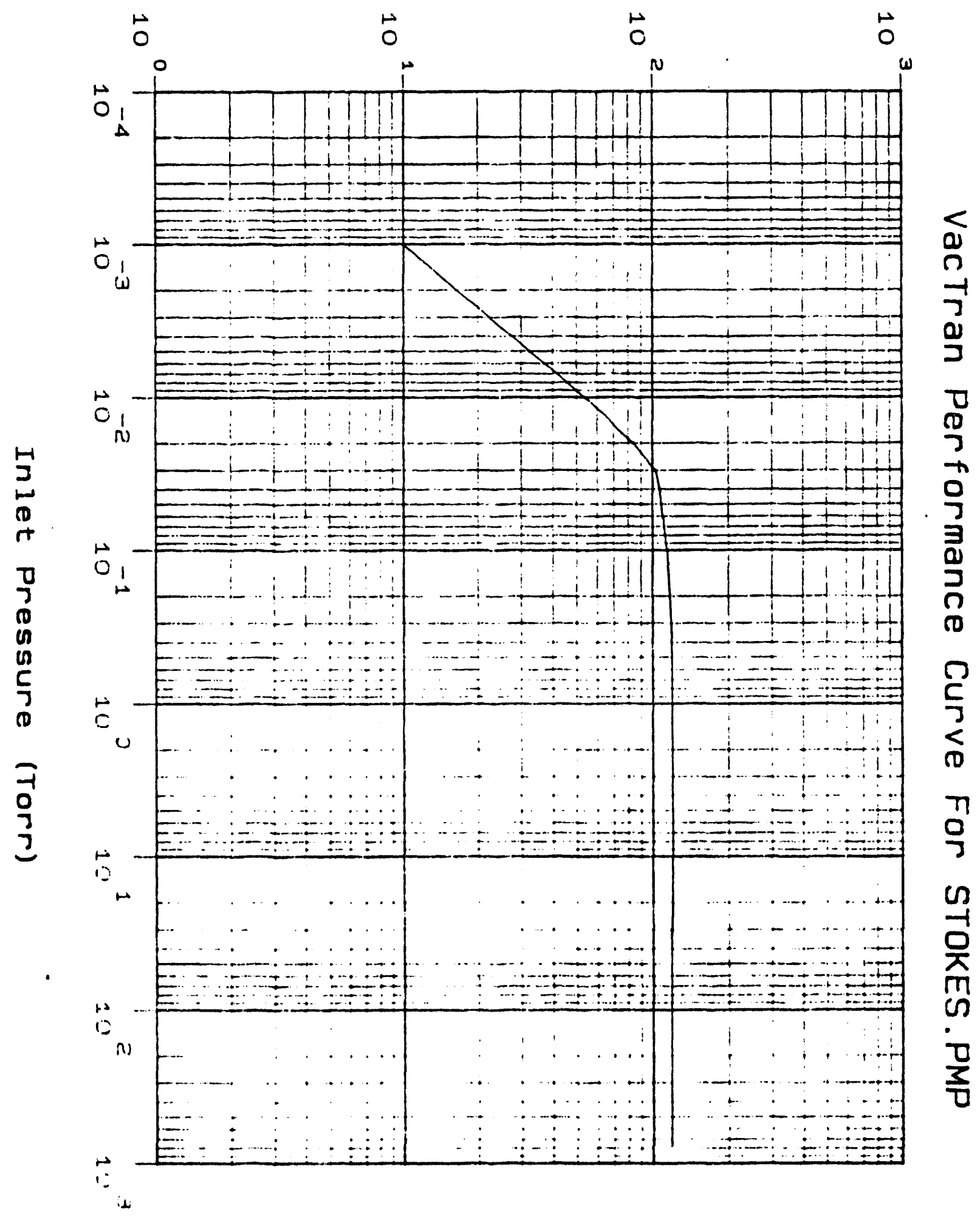

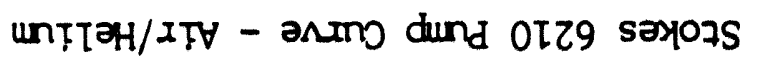


FIGURE B-9

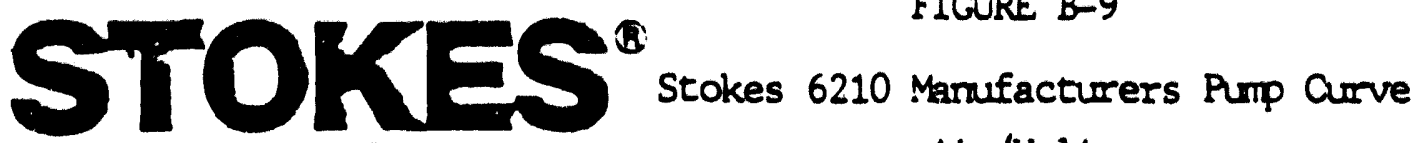
VACGLURM Air/Helium

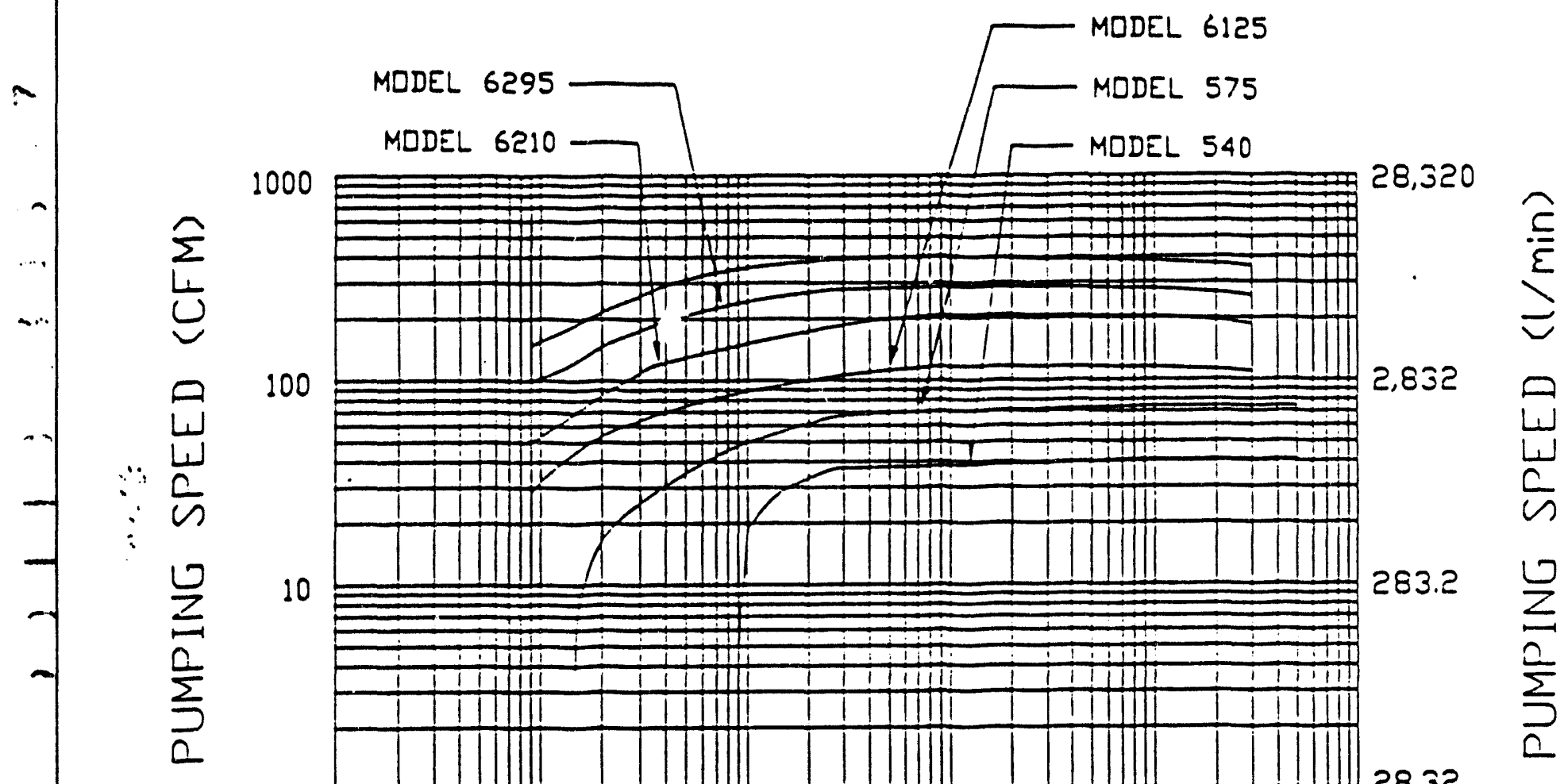
1 10

$10^{-2}$

$10^{-1}$

$10^{0}$

$10^{1}$

$10^{2}$

INLET PRESSURE (TORR)

PERFIRMANCE CURVES 
Vacuum System Analysis

Denise S Rohrdanz

JuTy 19, 1990

Appendix C

Conductance for the rotary piston pumps was based on total length of ducting from the pump to the vessel and all included bends and elbows. (Figure 7) The same conductance model was used for both the DK200 and the Balzers DU0250A. The parameters used are given below.

VacTran Conductance Model RUF.CND

Selected Gas = Air/Helium (Figures C-IA \& C-2A, respectively)

Current Temperature $=298.0$ Keivin

Conductance Start Pressure $=760.0$ Torr

Conductance Target Pressure $=1.000 \mathrm{E}-08$ Torr

1) Standard Elbow:

Diameter $=9.525$ Centimeters Angle $=90.0$ degrees

Elbow Volume $=0.2138$ Liters

2) Standard Elbow:

Diameter $=9.525$ Centimeters Angle $=45.0$ degrees

Elbow Volume $=0.4275$ Liters

3) Pipe Bend :

Bend Radius $=55.0$ Diameter $=9.525$ Centimeters Angle $=55.0$ degrees

Bend Volume $=1.042$ Liters

4) Cylindrical Pipe :

Diameter $=9.525$ Length $=528.8$ Centimeters

Pipe Volume $=37.68$ Liters

VacTran Conductance Model 6INCOND.CND

Selected Gas - Air/Helium (Figures C-1B \& C-2B, respectively)

Current Temperature $=298.0$ Kelvin

Conductance Start Pressure $=760.0$ Torr

Conductance Target Pressure $=1.000 \mathrm{E}-08$ Torr

1) Standard Elbow:

Diameter $=14.6$ Centimeters Angle $=90.0$ degrees

Elbow Volume $=0.5026$ Liters

2) Standard Elbow:

Diameter $=14.6$ Centimeters Angle $=45.0$ degrees

Elbow Volume $=1.005$ Liters

3) Cylindrical Pipe :

Diameter $=14.6$ Length $=528.8$ Centimeters

Pipe Volume $=88.59$ Liters

4) Pipe Bend :

Bend Radius $=55.0$ Diameter $=14.6$ Centimeters Angle $=55.0$ degrees

Bend Volume $=3.522$ Liters 


\section{Appendix C}

VacTran Conductance Model 8INCOND

Selected Gas = Air/Helium (Figures C-1C \& C-2C, respectively)

Current Temperature $=298.0$ Kelvin

Conductance Start Pressure $=760.0$ Torr

Conductance Target Pressure $=1.000$ E-08 Torr

1) Standard Elbow :

Diameter $=20.32$ Centimeters Angle $=90.0$ degrees

Elbow Volume $=0.9729$ Liters

2) Standard Elbow :

Diameter $=20.32$ Centimeters Angle $=45.0$ degrees

Elbow Volume $=1.946$ Liters

3) Cylindrical Pipe :

Diameter $=20.32$ Length $=528.8$ Centimeters

Pipe Volume $=171.5$ Liters

4) Pipe Bend :

Bend Radius $=55.0$ Diameter $=20.32$ Centimeters Angle $=55.0$ degrees Bend Volume $=9.488$ Liters

Conductance for the turbomolecular pumps was assumed constant at:

- 2,226 liters/sec for air

- 5,889 liters/sec for helium 
FIGURE C-1A

Rough Pump Conctuctance - Air
4-inch duct

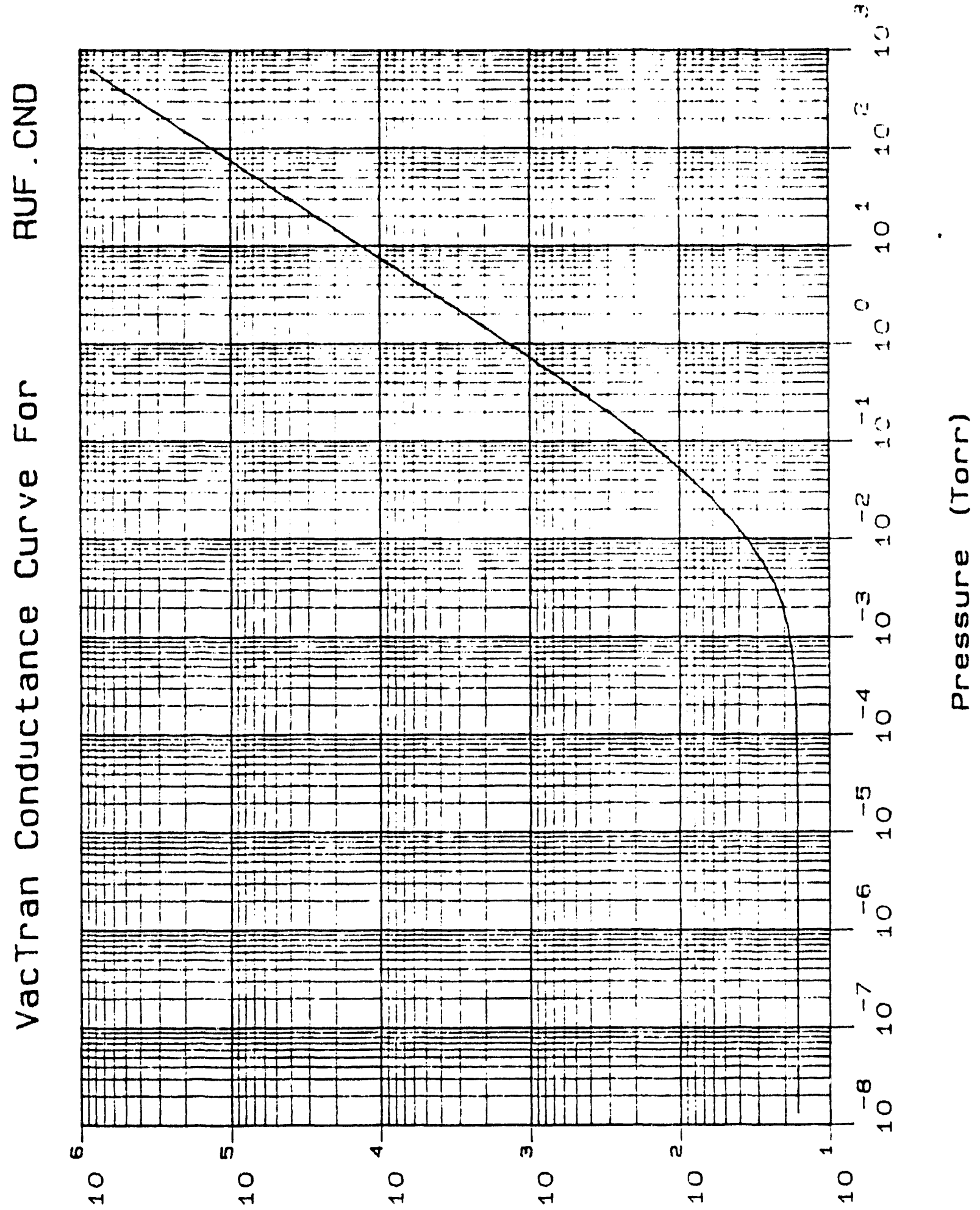

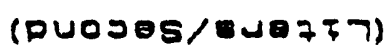

aวue z כกpบoכ 
S LGUKt $U-10$

Rough Pump Conductance - Air

6-inch duct

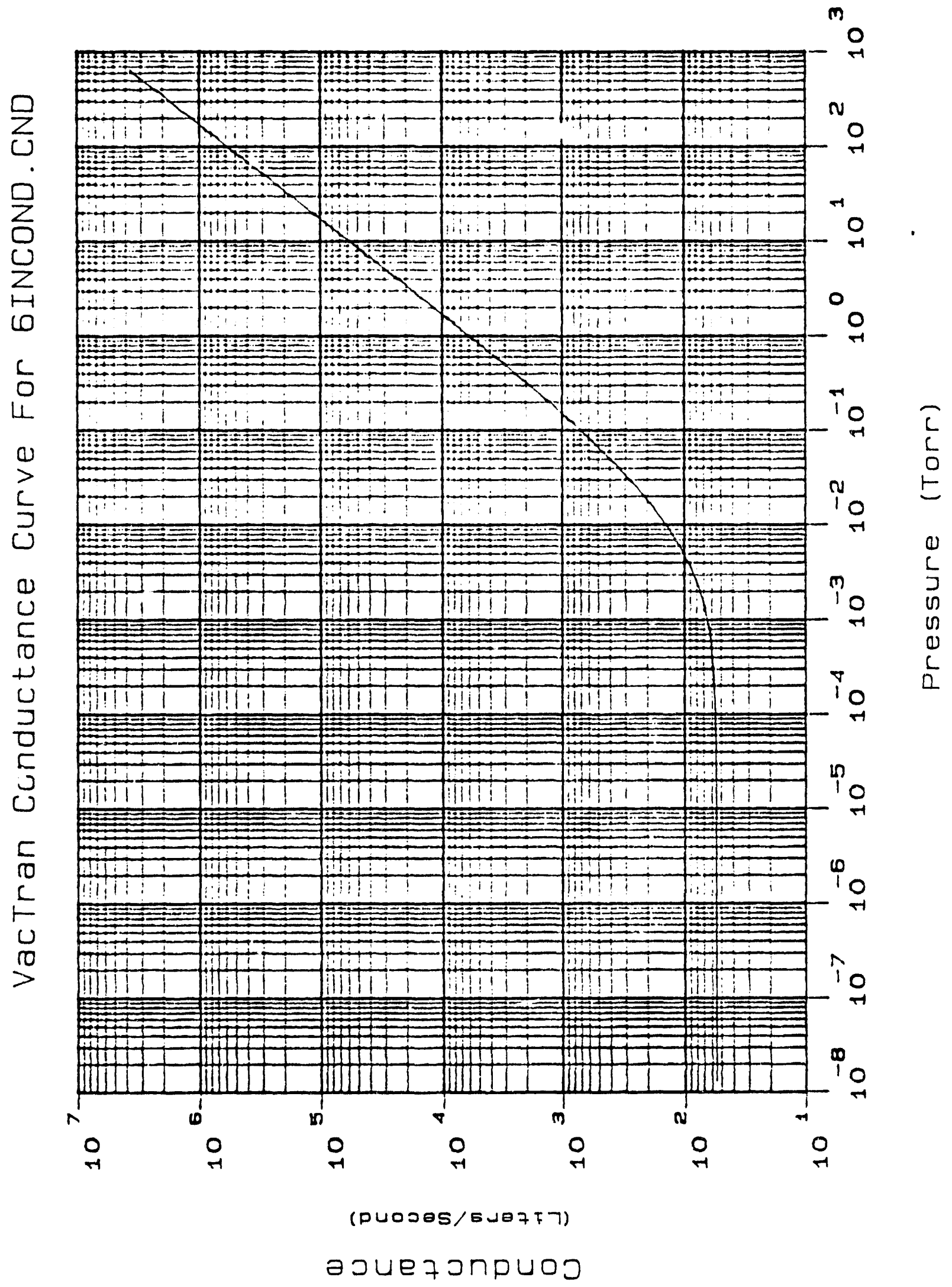


FIGURE C-1C

Rough Pump Conductance - Air

8-inch duct

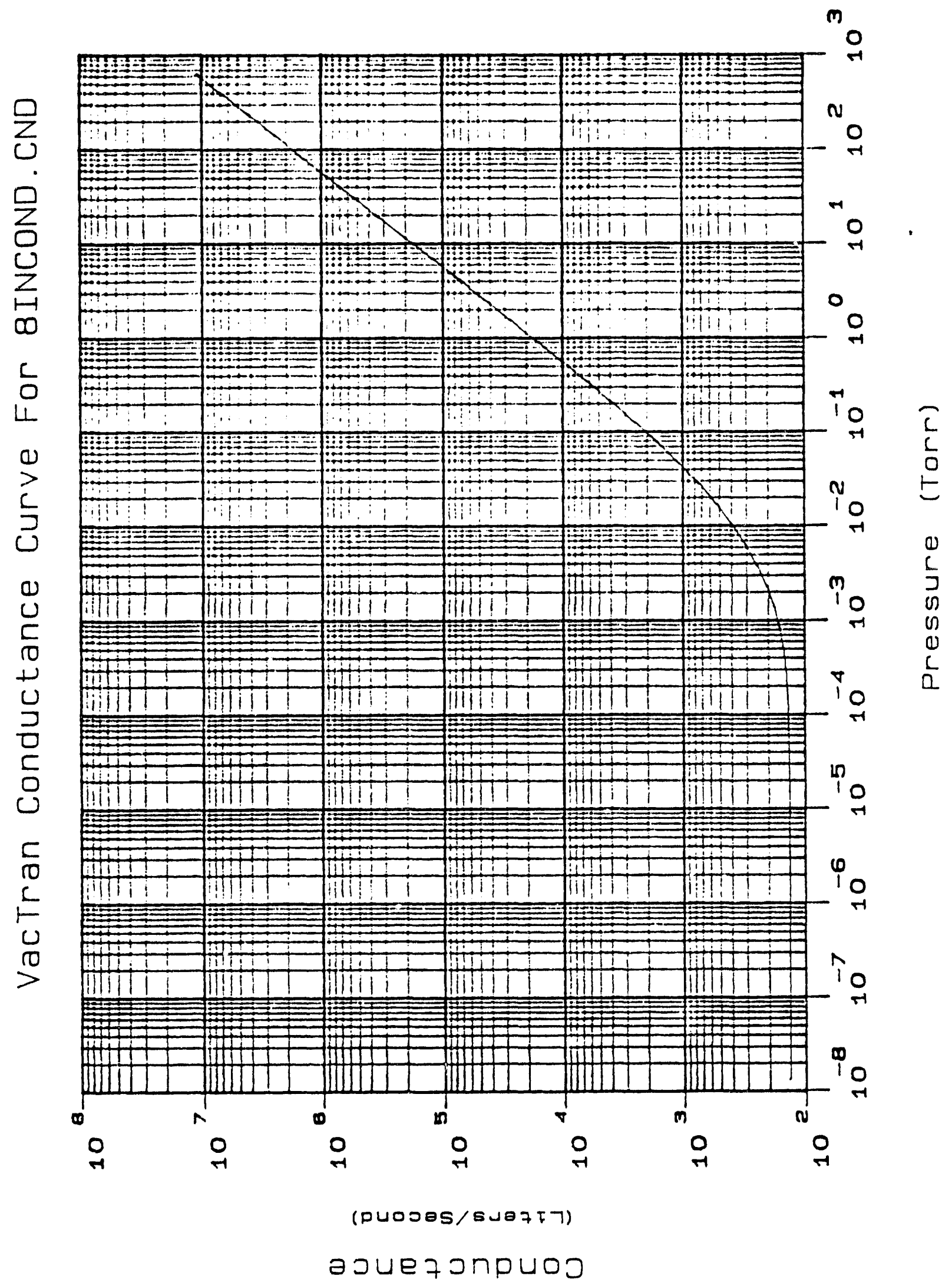


FIGURE C-2A

Rough Pump Conductance - Helium

4-inch duct

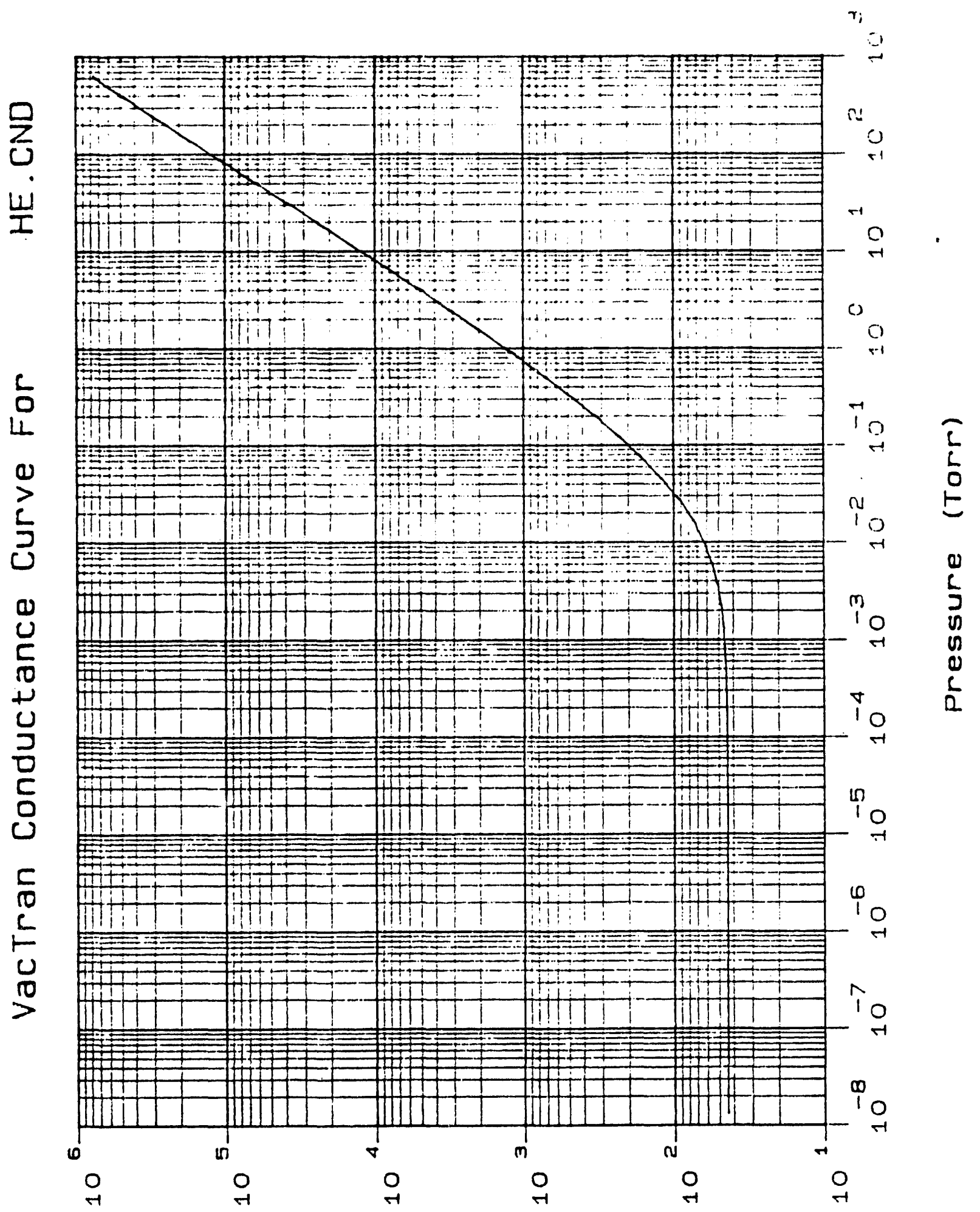

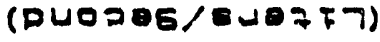

a כUe 
FIGURE C-2B

Rough Pump Conductance - Helium

6-inch duct

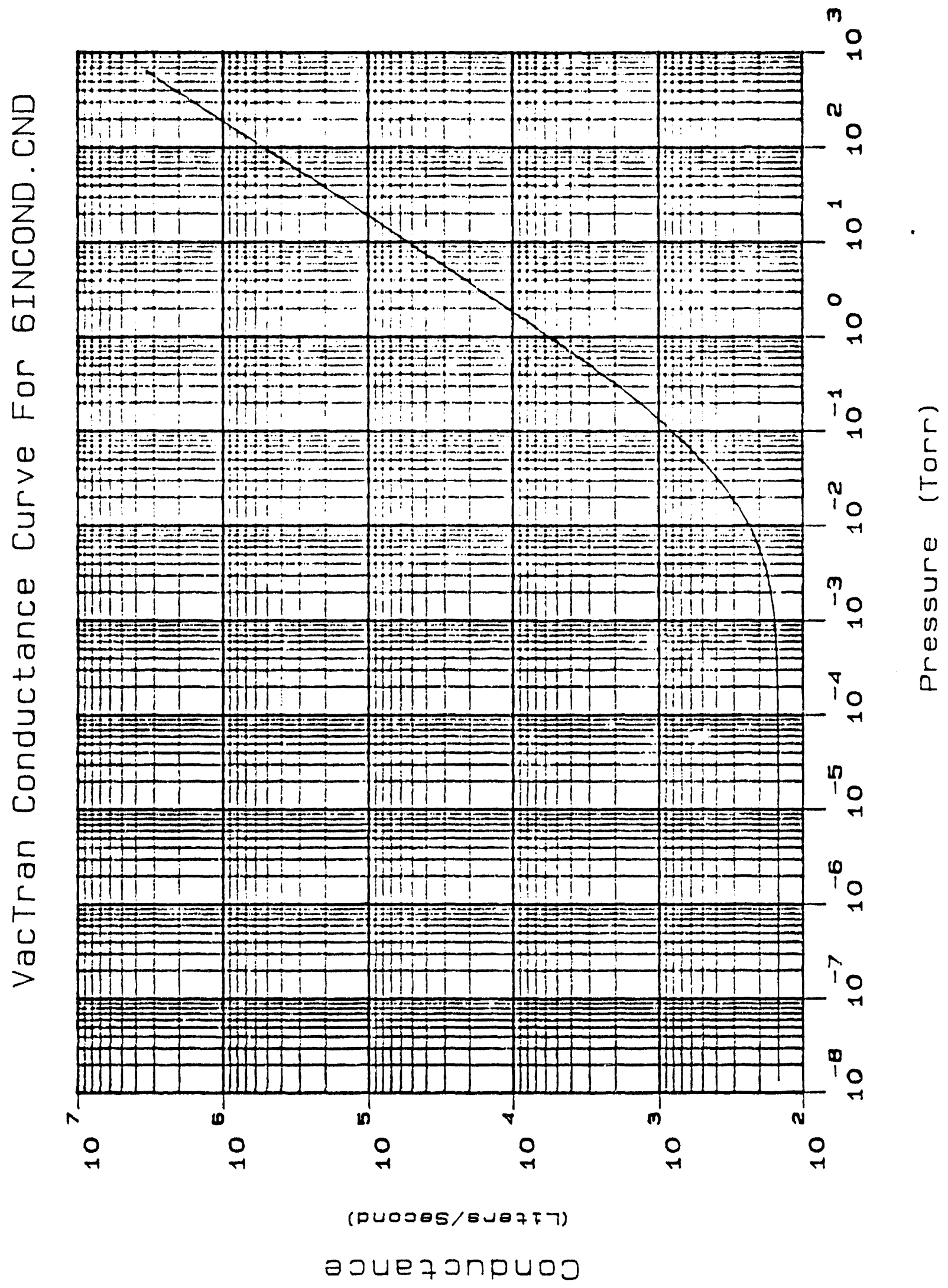


FIGURE C-2C

Rough Pump Conductance - Helium

8-inch duct

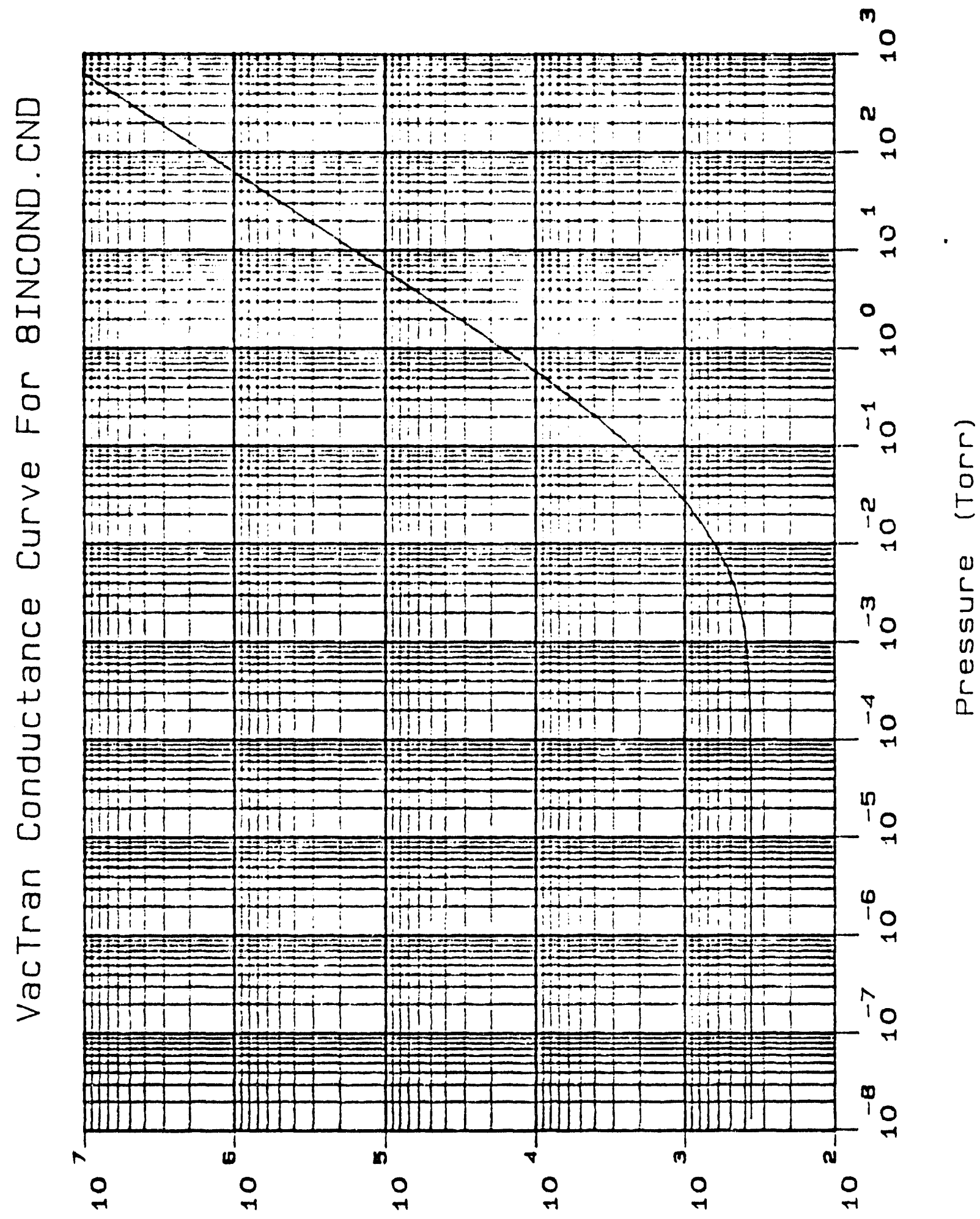

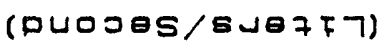

aวueq onpuoJ 


\section{Vacuum System Analysis}

Denise S Rohrdanz

July 19,1990

Appendix 0

At equilibrium, the permeation rate is given as

$$
\begin{aligned}
& q=\underline{K}_{p} \frac{P A}{d} \quad\left(0^{\prime} \text { Hanion, } 130\right) \\
& \text { where: } d=\text { wall thickness }(1.37 \mathrm{~cm}) \\
& P=\text { pressure difference across wall, } \\
& \text { for hydrogen } \\
& P=P_{\text {atmosphere }} \text { - Pyessel } \\
& P \text { atmosphere }=3.825 \mathrm{E}-4 \text { torr }\left(0^{\prime} \text { Hanton, } 4\right) \\
& P_{\text {vessel }}=1 E-8 \text { torr, design } \\
& K_{P}=\text { permeability constant }\left(\mathrm{cm}^{2} / \mathrm{sec}\right) \\
& A=\text { inside surface area }\left(7.8 E+5 \mathrm{~cm}^{2}\right) \\
& K_{p}=D S^{\prime} \quad\left(0^{\prime} \operatorname{Han} 7\right. \text { on, 130) } \\
& \text { where: } S^{\prime}=1 \text { (solid solubility at steady-state) } \\
& D=\text { diffusion constant } \\
& D=D_{0} \exp \left(-E_{d} / R T\right) \text { (Kass \& Andrzewski: Perkins) } \\
& \text { where: } D_{0}=.012 \mathrm{~cm}^{2} / \mathrm{sec} \text { (for } 300 \text { series stainless } \\
& E_{d}=13,100 \mathrm{cal} / \mathrm{g}-\mathrm{mol} \text { (for } 300 \text { series } \\
& \text { stainless steel) } \\
& R=1.987 \mathrm{cal} / \mathrm{g}-\mathrm{mol}-\mathrm{K} \\
& T=\text { temperature }(K)
\end{aligned}
$$

for $25^{\circ} \mathrm{C}$

$D=\left(.012 \mathrm{~cm}^{2} / \mathrm{sec}\right) \exp [(-13,100 \mathrm{cal} / \mathrm{g}-\mathrm{mol}) /(1.987 \mathrm{cal} / \mathrm{g}-\mathrm{mol}-\mathrm{K} * 298 \mathrm{~K})]$

$D=2.96 \mathrm{E}-12 \mathrm{~cm}^{2} / \mathrm{sec}$

$q=\left[\left(2.958 E-12 \mathrm{~cm}^{2} / \mathrm{sec}\right)(3.825 E-4\right.$ torr $-1 E-8$ torr $\left.) / 1.37 \mathrm{~cm}\right] 7.8 E+5 \mathrm{~cm}^{2}$

$q=6.44 E-10$ torr -1 iters $/ \mathrm{sec}$

for $93^{\circ} \mathrm{C}$

$D=\left(.012 \mathrm{~cm}^{2} / \mathrm{sec}\right) \exp [(-13,100 \mathrm{cal} / \mathrm{g}-\mathrm{mol}) /(1.987 \mathrm{cal} / \mathrm{g}-\mathrm{mol}-\mathrm{K} \star 365 \mathrm{~K})]$

$D=1.80 \mathrm{E}-10 \mathrm{~cm}^{2} / \mathrm{sec}$

$q=\left[\left(1.8 E-10 \mathrm{~cm}^{2} / \mathrm{sec}\right)(3.825 E-4\right.$ torr $-1 E-8$ torr $\left.) / 1.37 \mathrm{~cm}\right] 7.8 E+5 \mathrm{~cm}^{2}$

$q=3.92 E-8$ tor -7 iters $/ \mathrm{sec}$ 


\section{Vacuum System Analysis} Denise $S$ Rohrdanz July 19, 1990

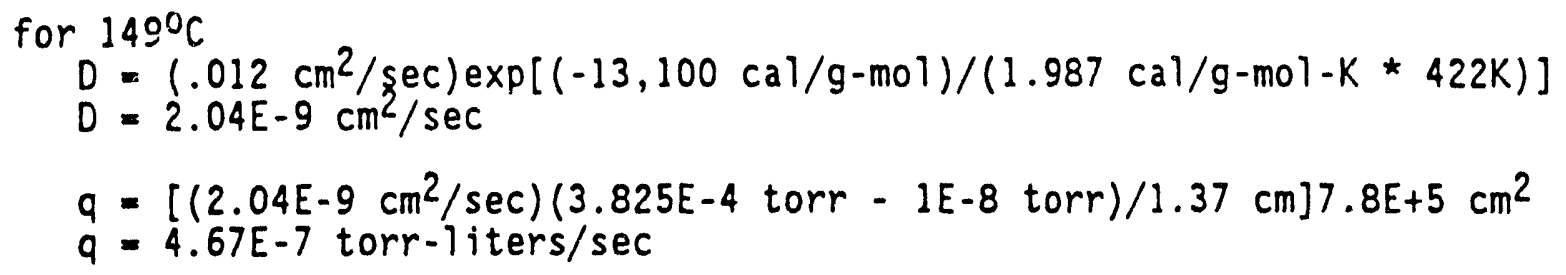




\section{Pump Down Curve for RUF.SYS}

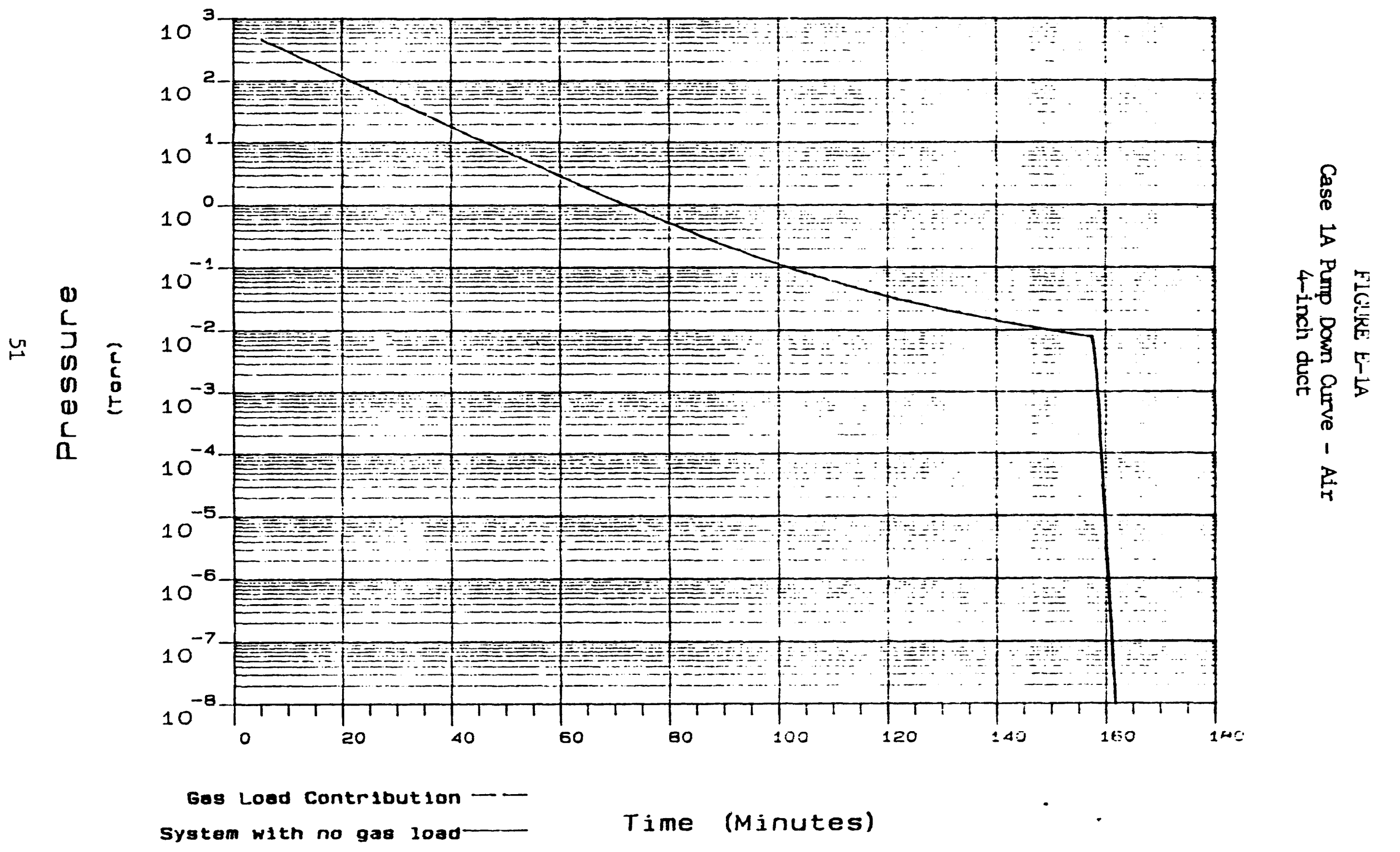


Pump Down Curve for RUF.SYS

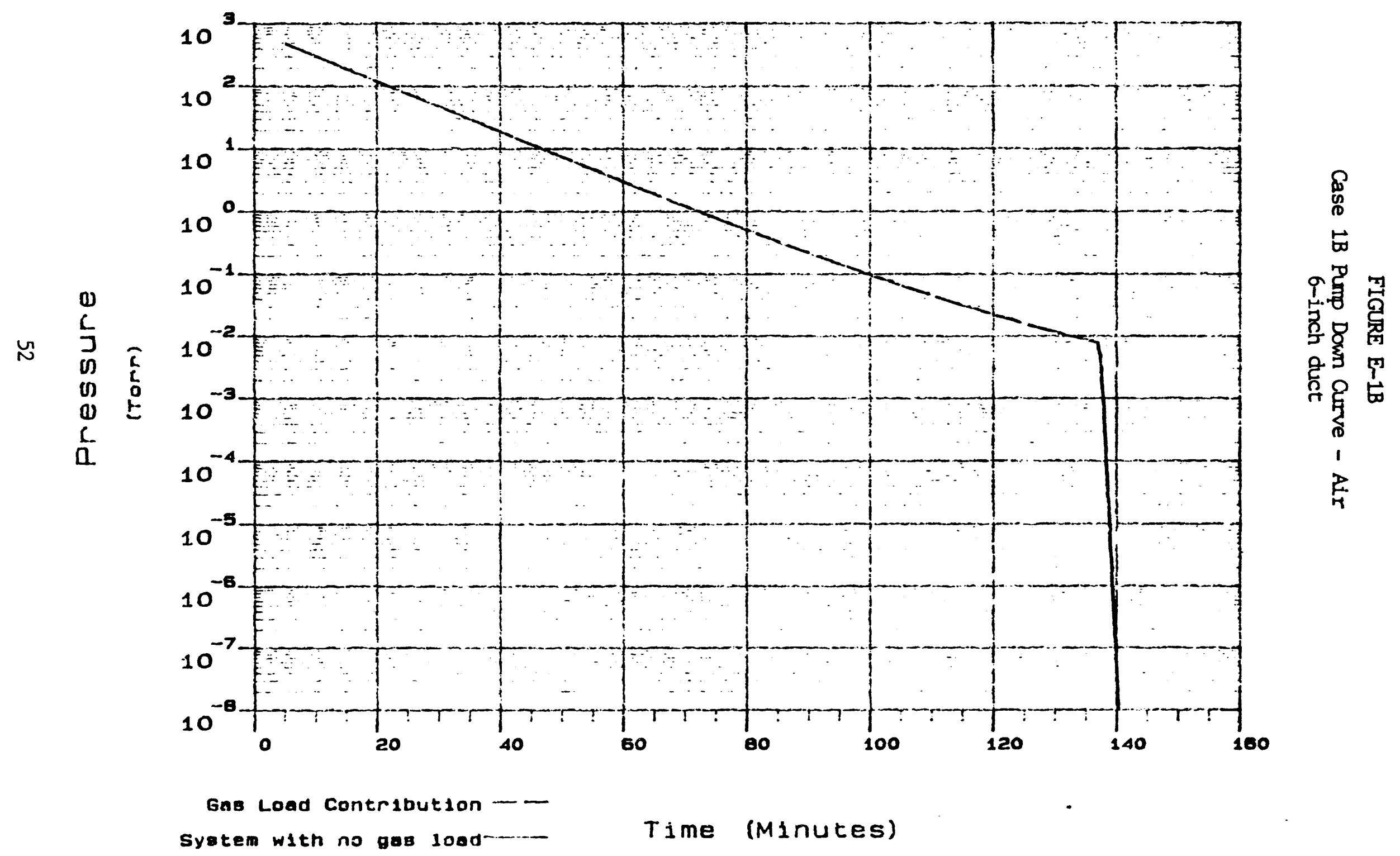




\section{Pump Down Curve for RUF.SYS}

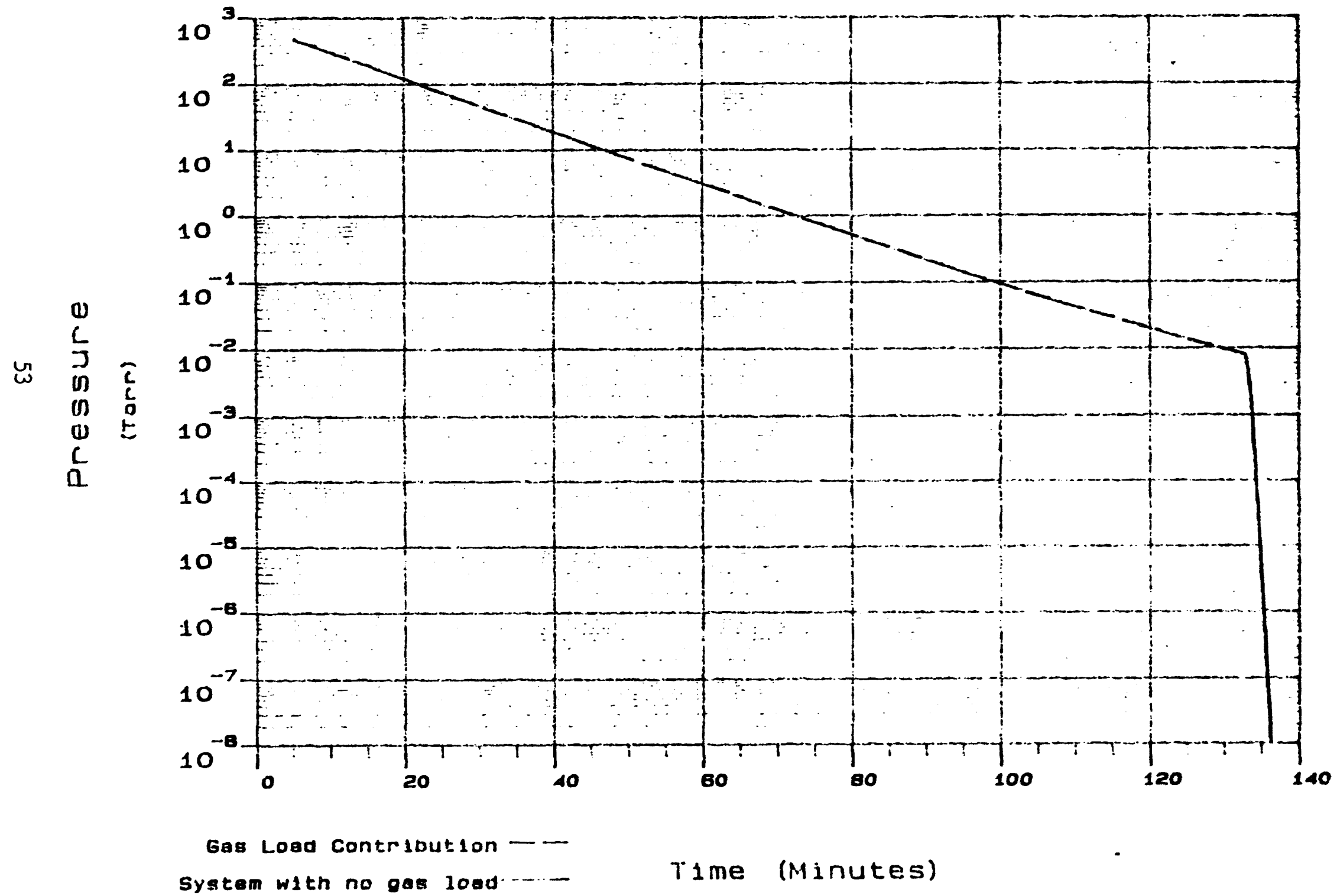




\section{Pump Down Curve for AF2.SYS}

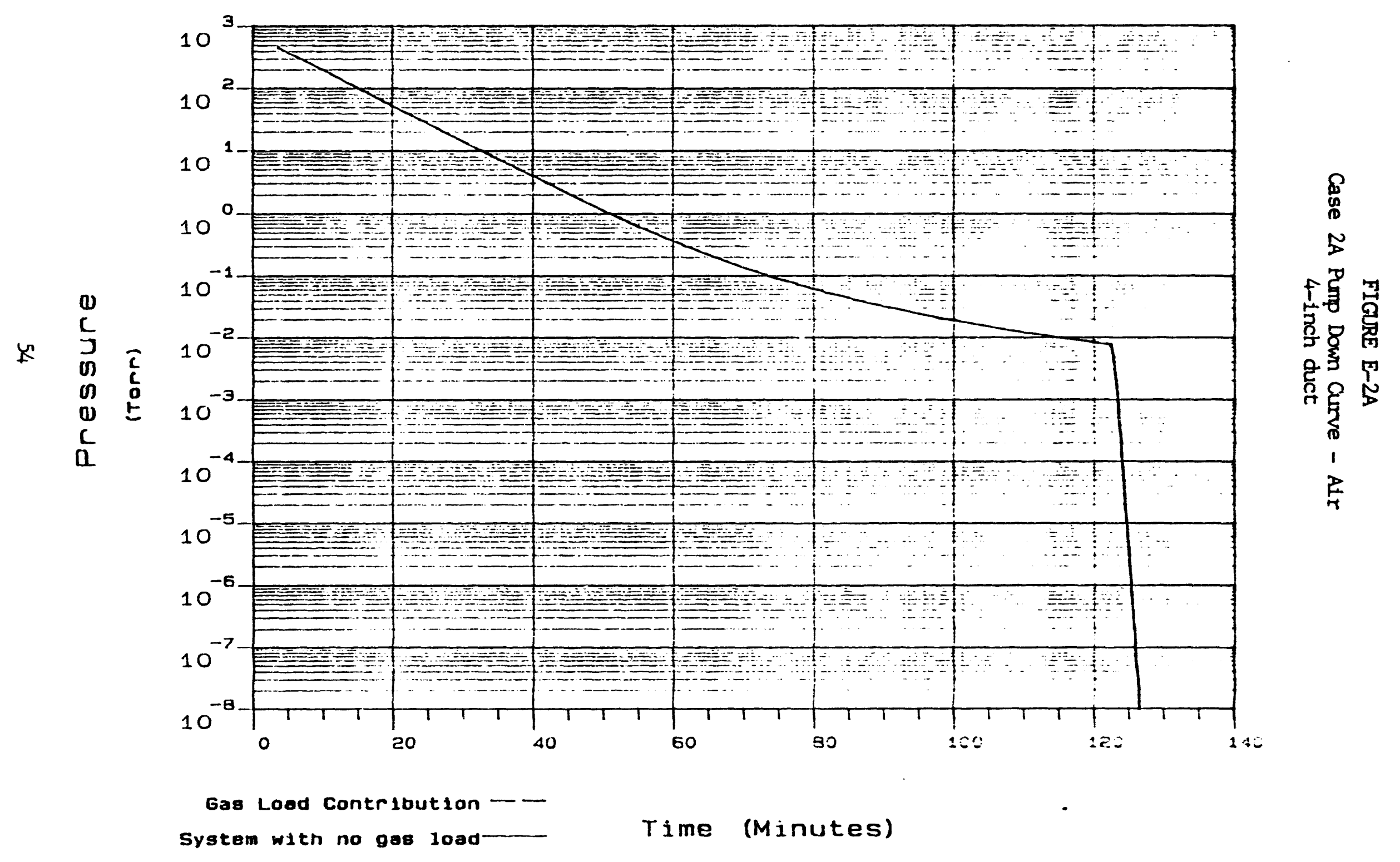


, 1 ! : ; ;

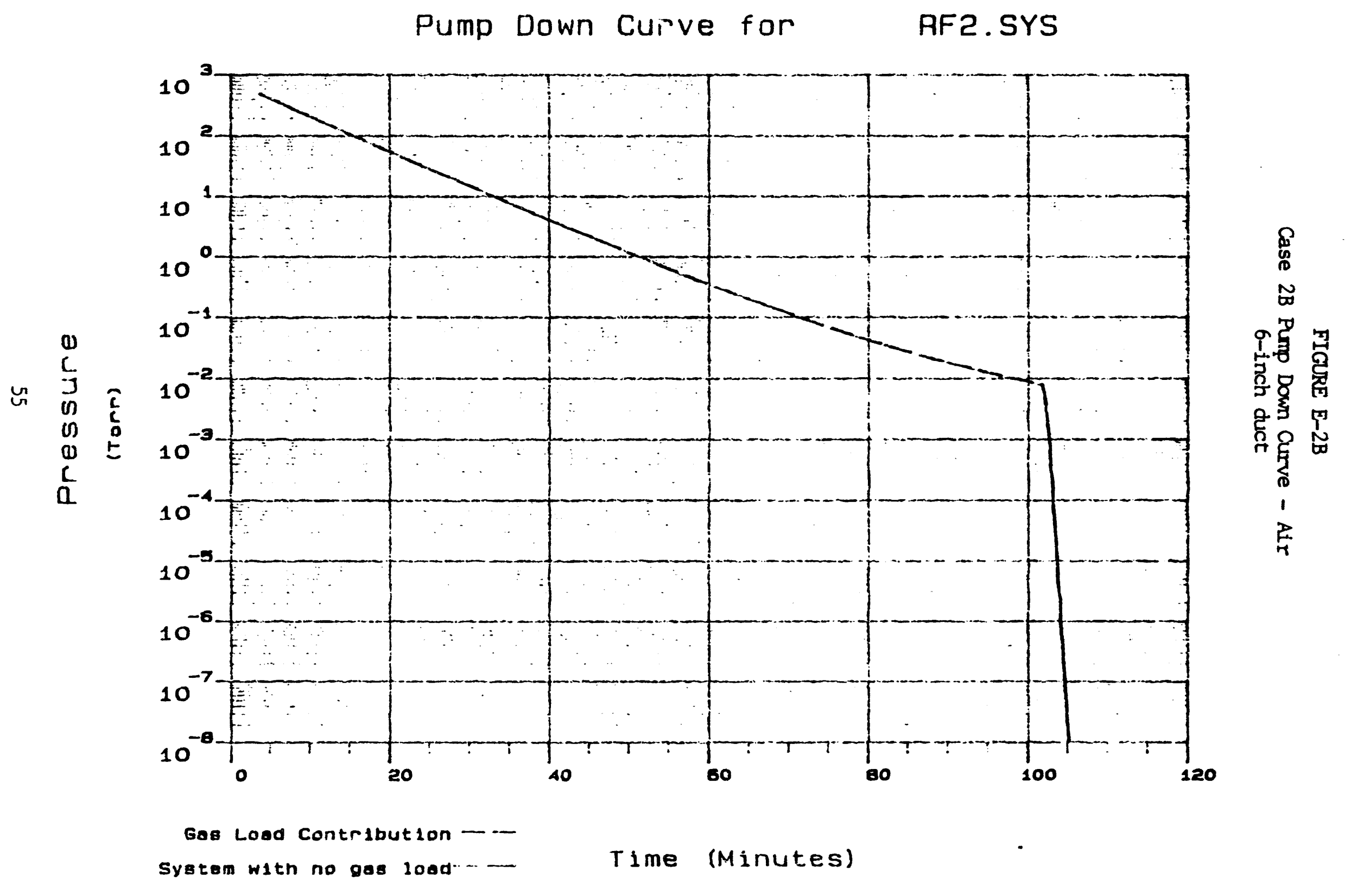


FIGURE E-2C

Case 2C Pump Down Curve - Air

8-inch duct

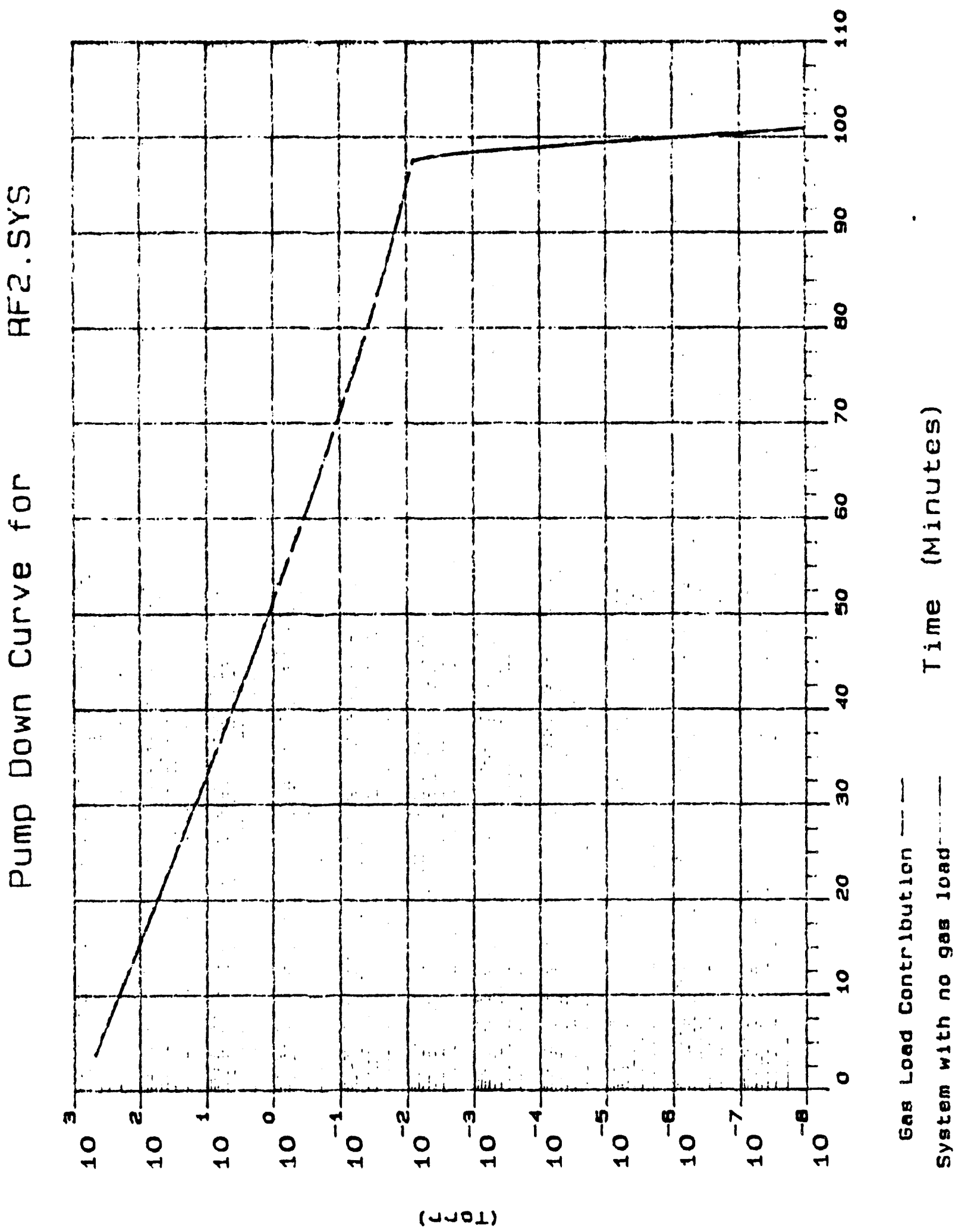

aunssaud 
(saznuț) aแโ 1

poot 806 ou H7fM we7sKs

_- uorzngtufuoj peot seg

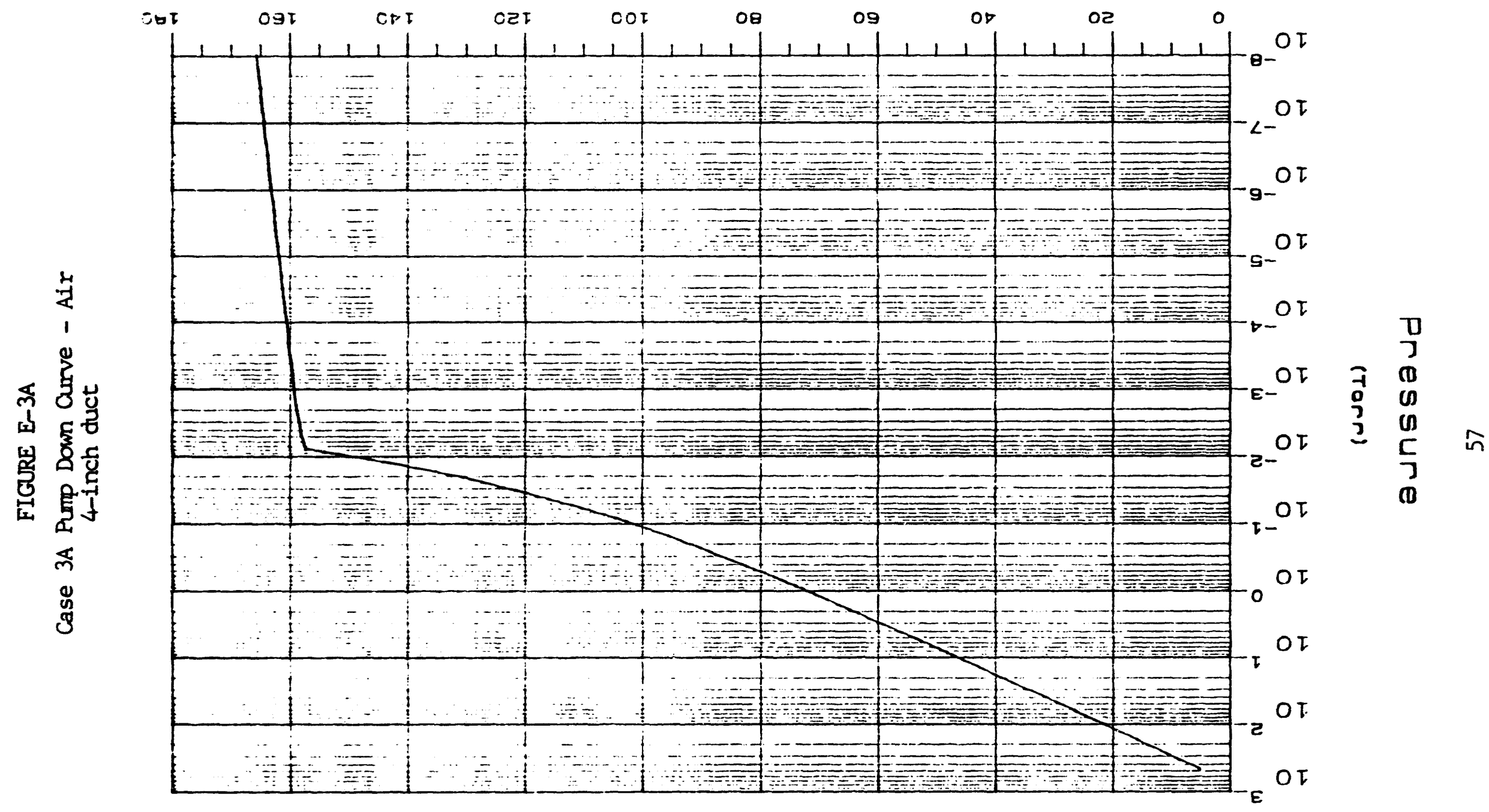

$\left.\mathrm{S}^{\prime} \mathrm{S}^{\circ} \varepsilon\right\lrcorner H$

Jof anung uMOg dwnd 


\section{Pump Down Curve for}

RF3.SYS

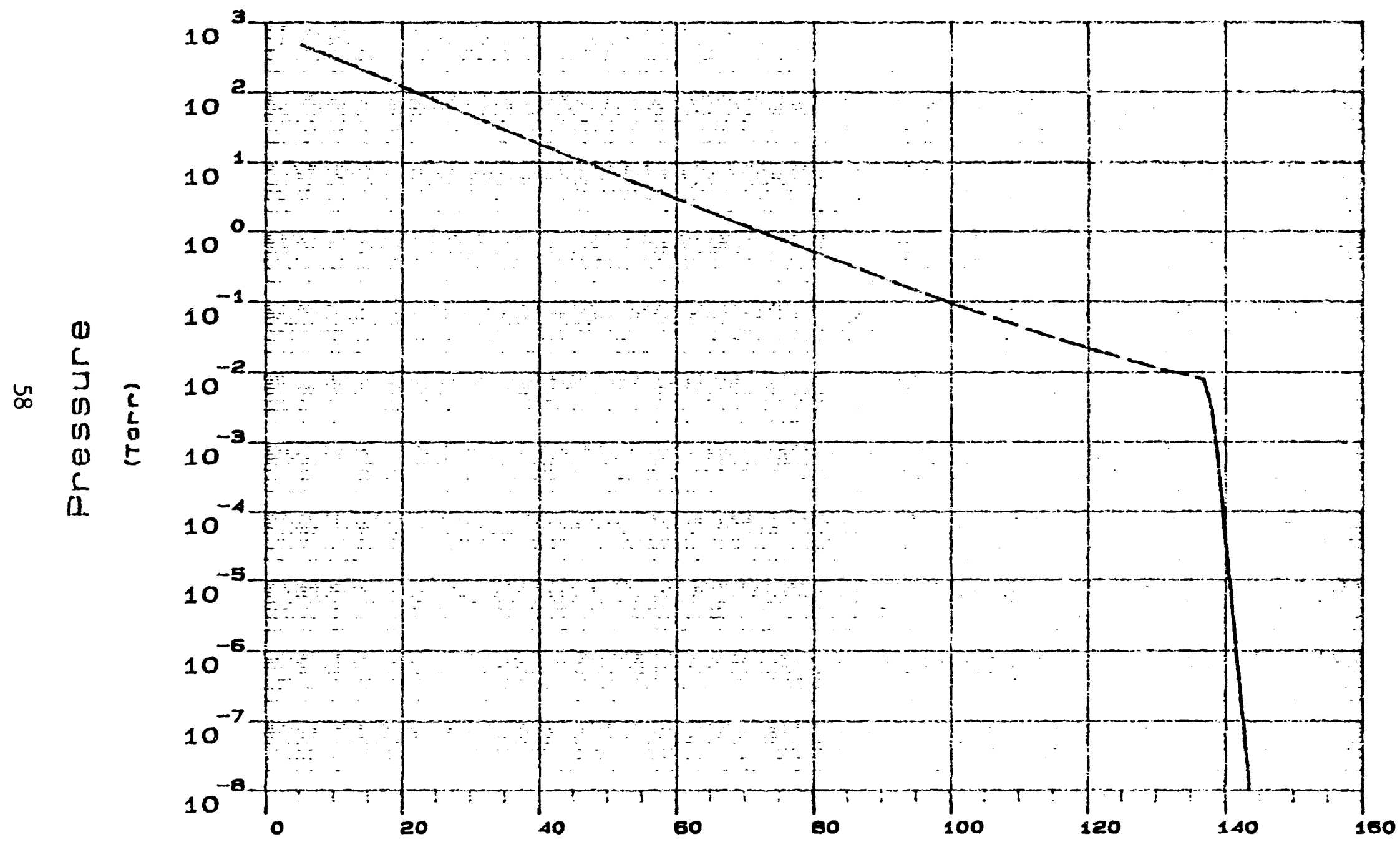

Gas Losd Contribution - - 
Pump Down Curve for

AF 3.SYS

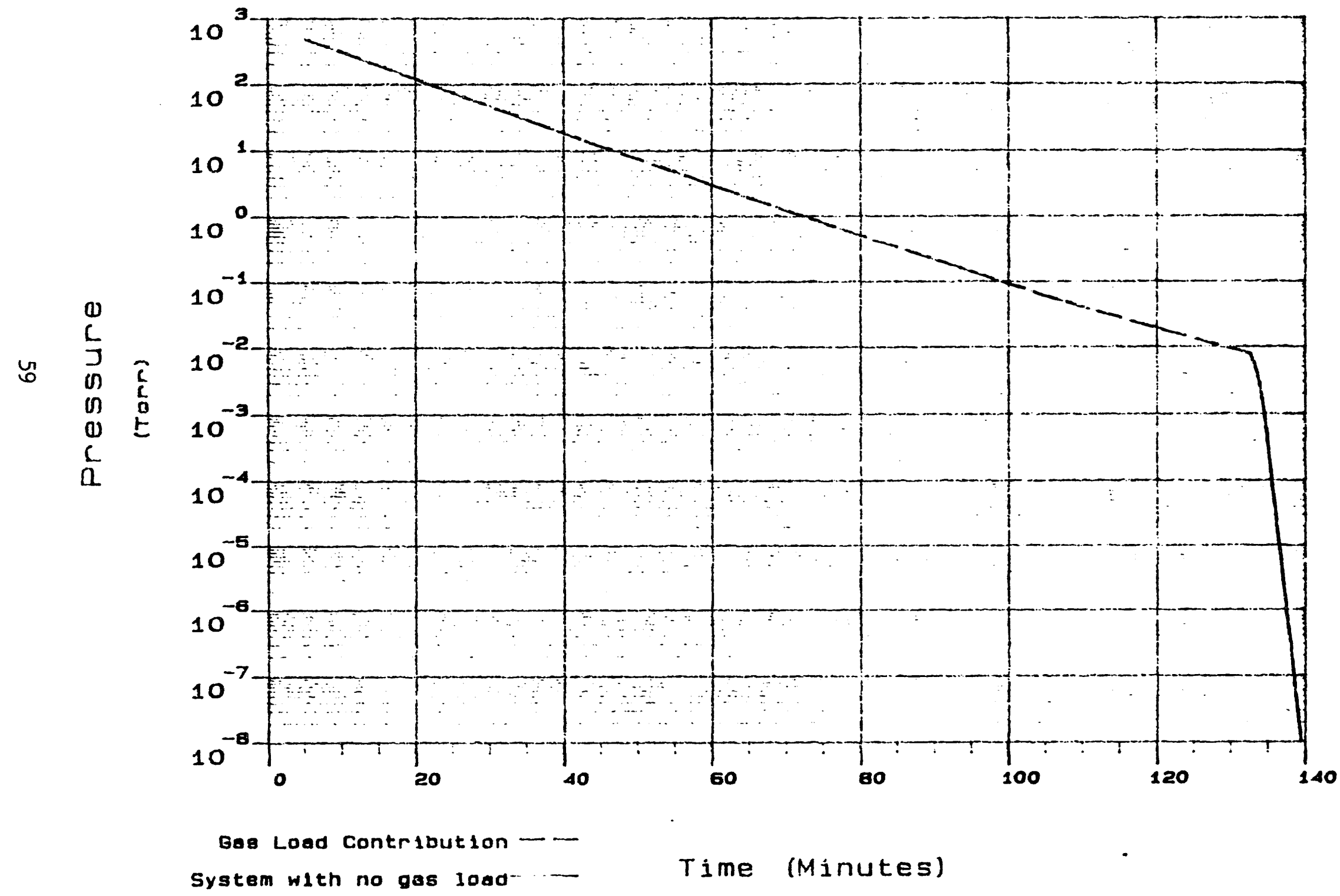


Pump Down Curve for STOKES.SYS

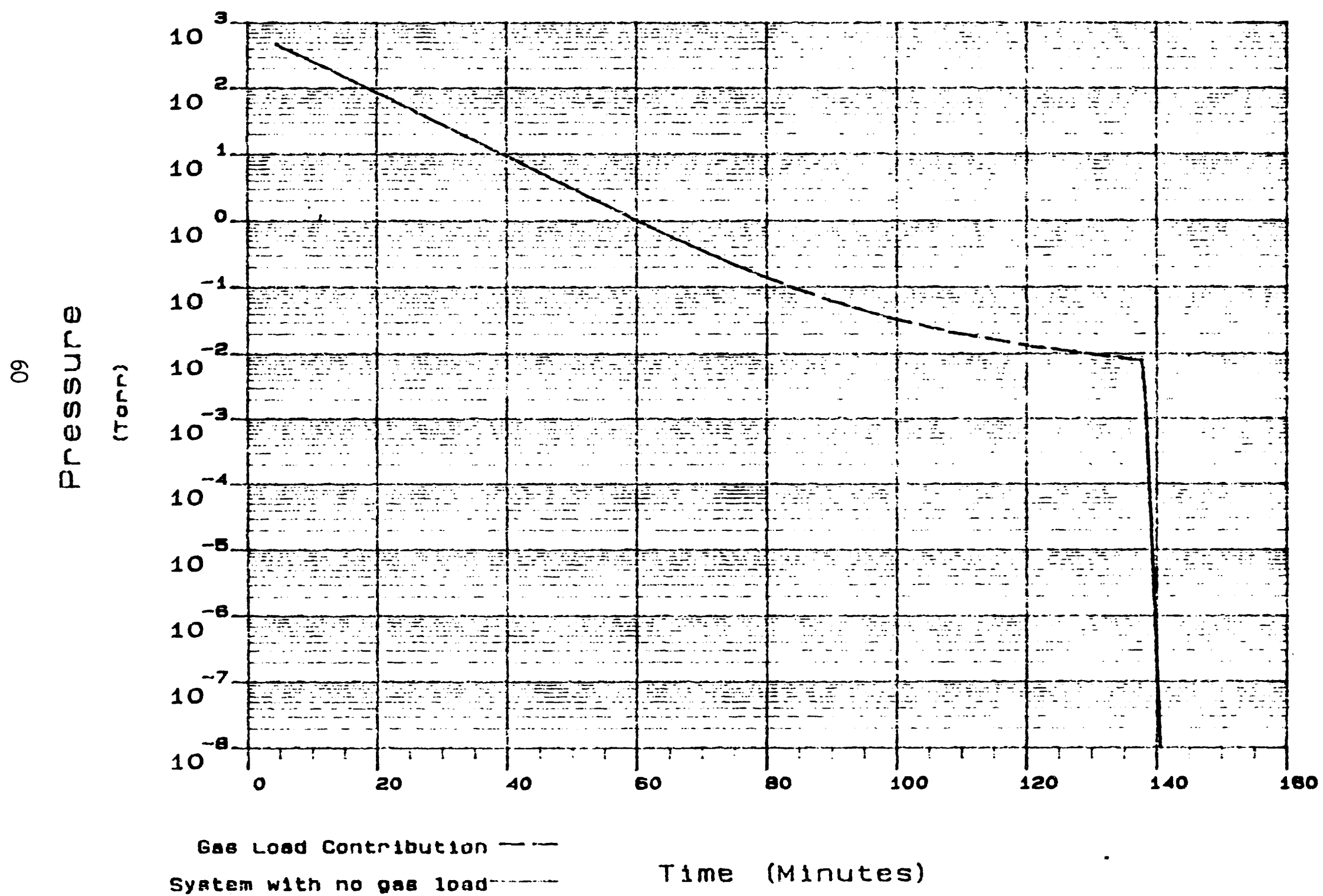


(sa7nบโพ) จงเ 1

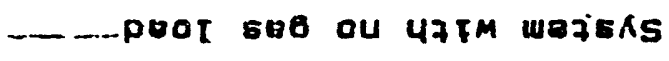

_... UoringtdzU0J p807 geg

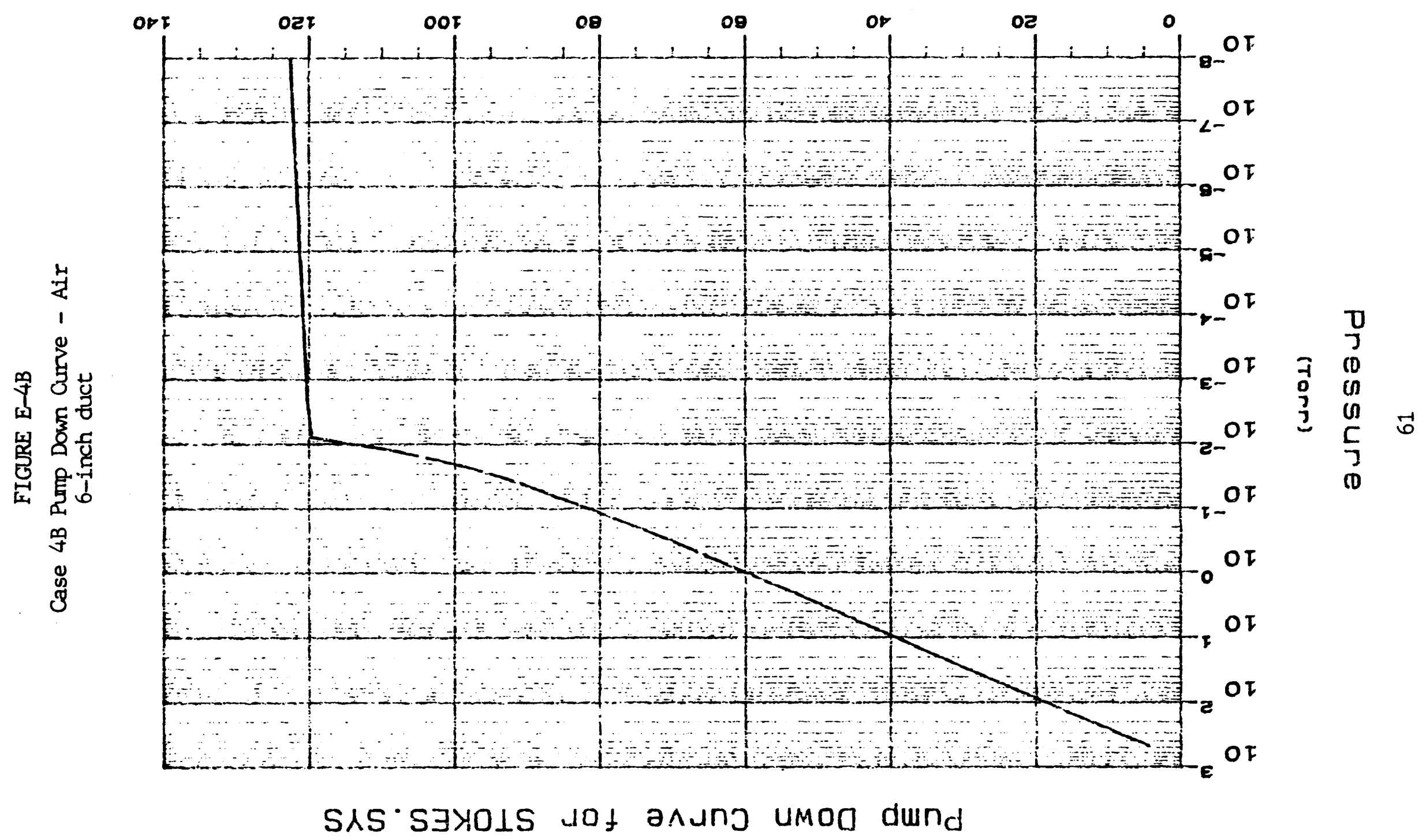


Pump Down Curve for STOKES.SYS

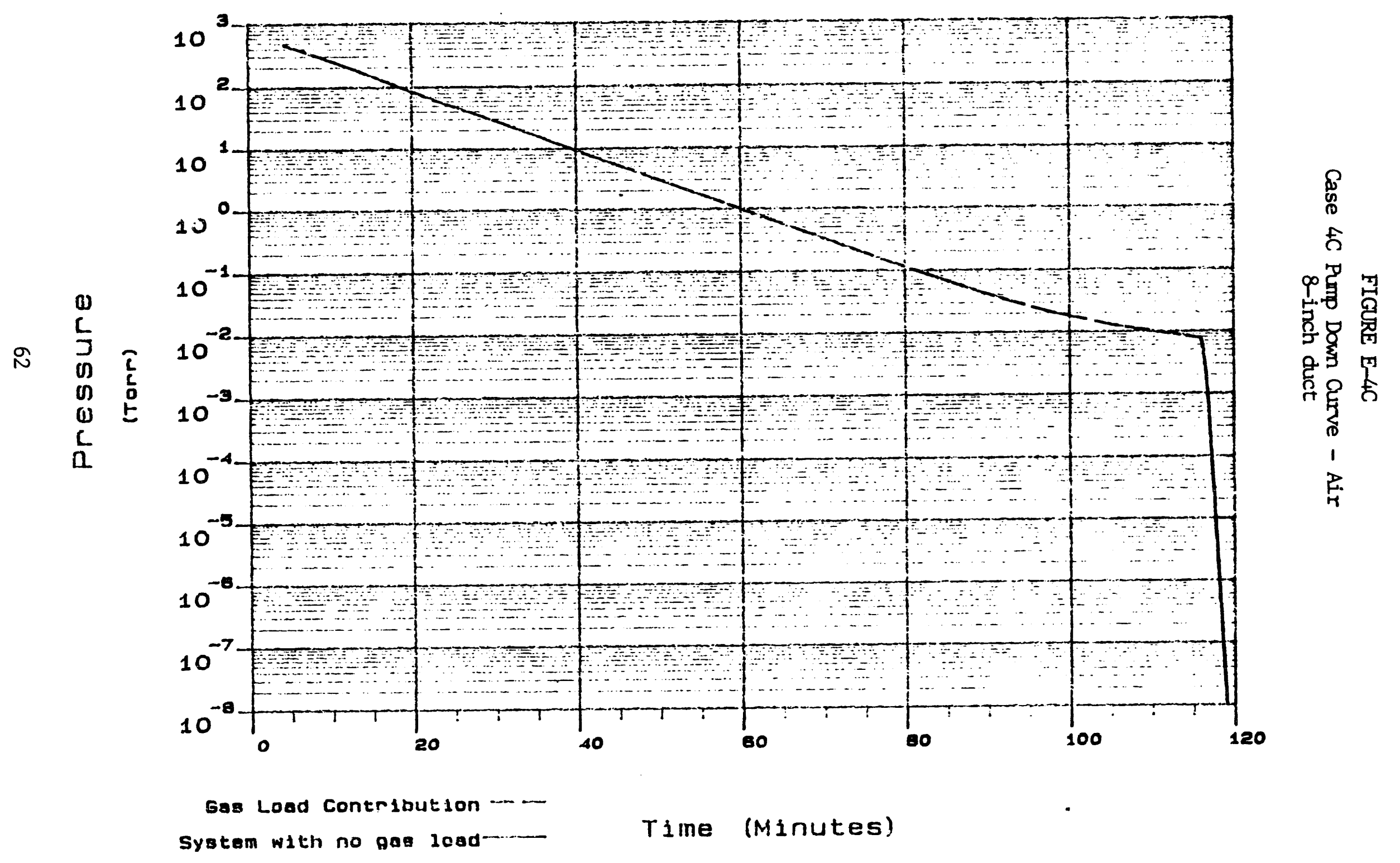




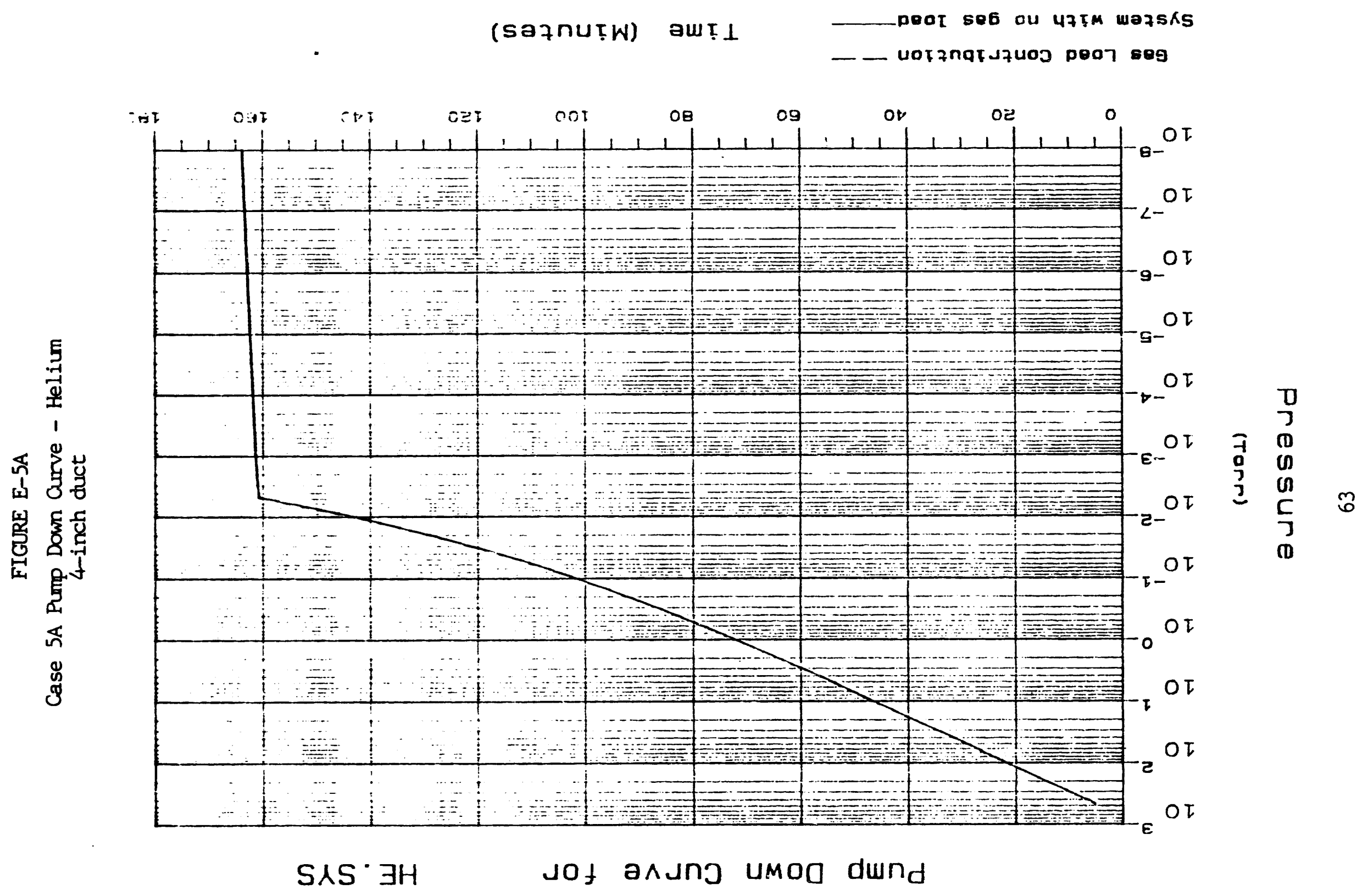


FIGURE E-5B

Case 5B Pump Down Curve - Helium

6-inch duct

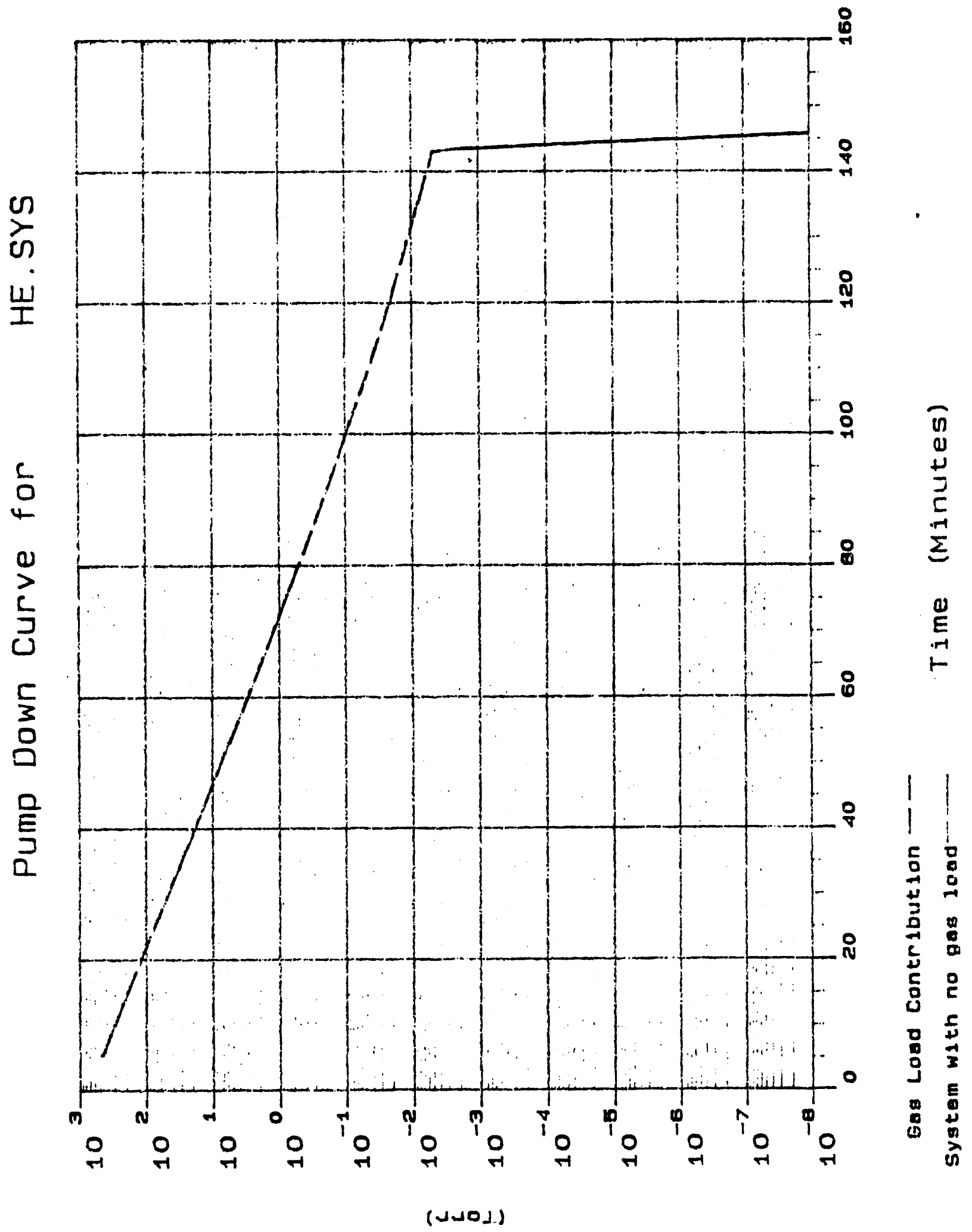

aunssaud 
Pump Down Curve for

HE.SYS

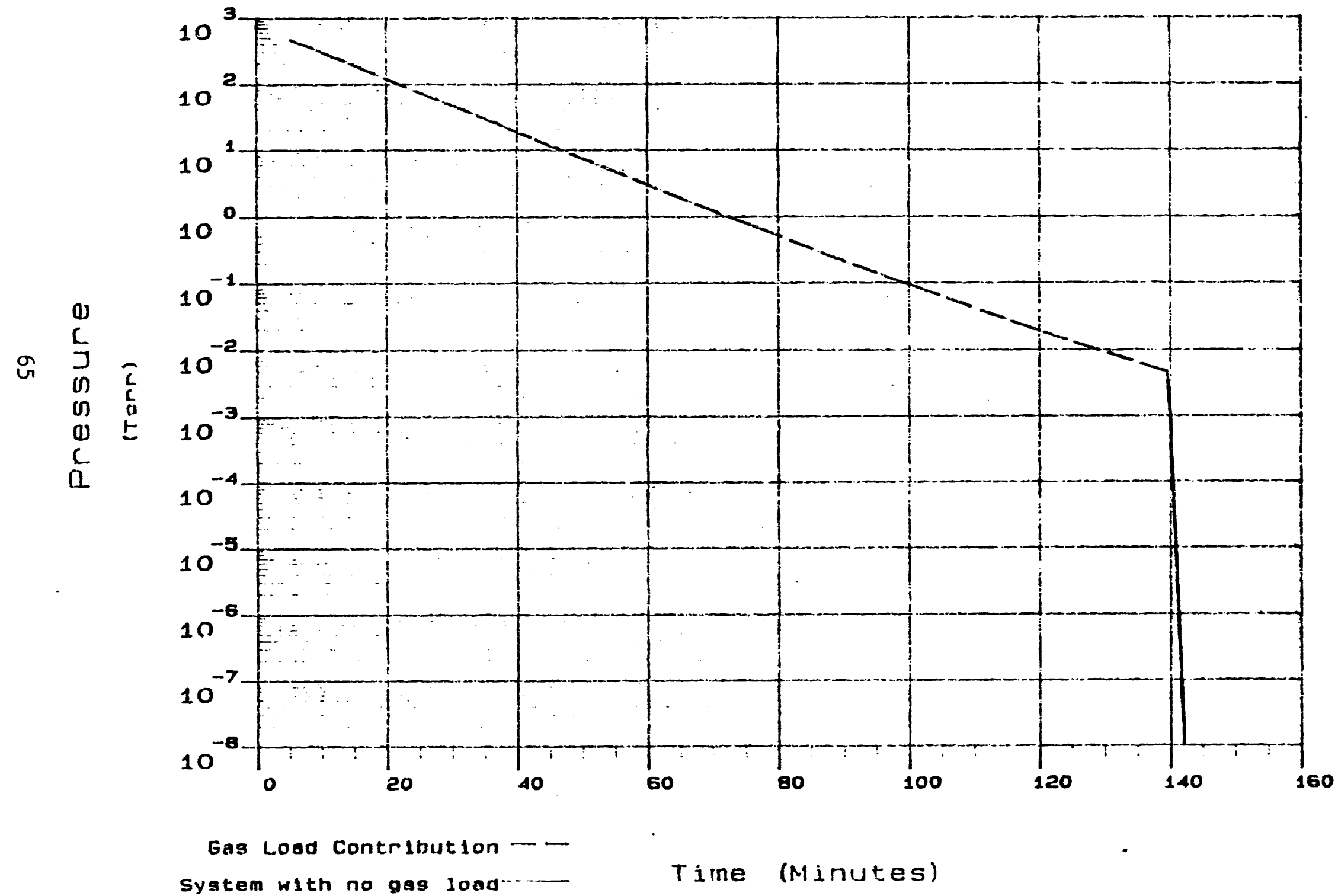



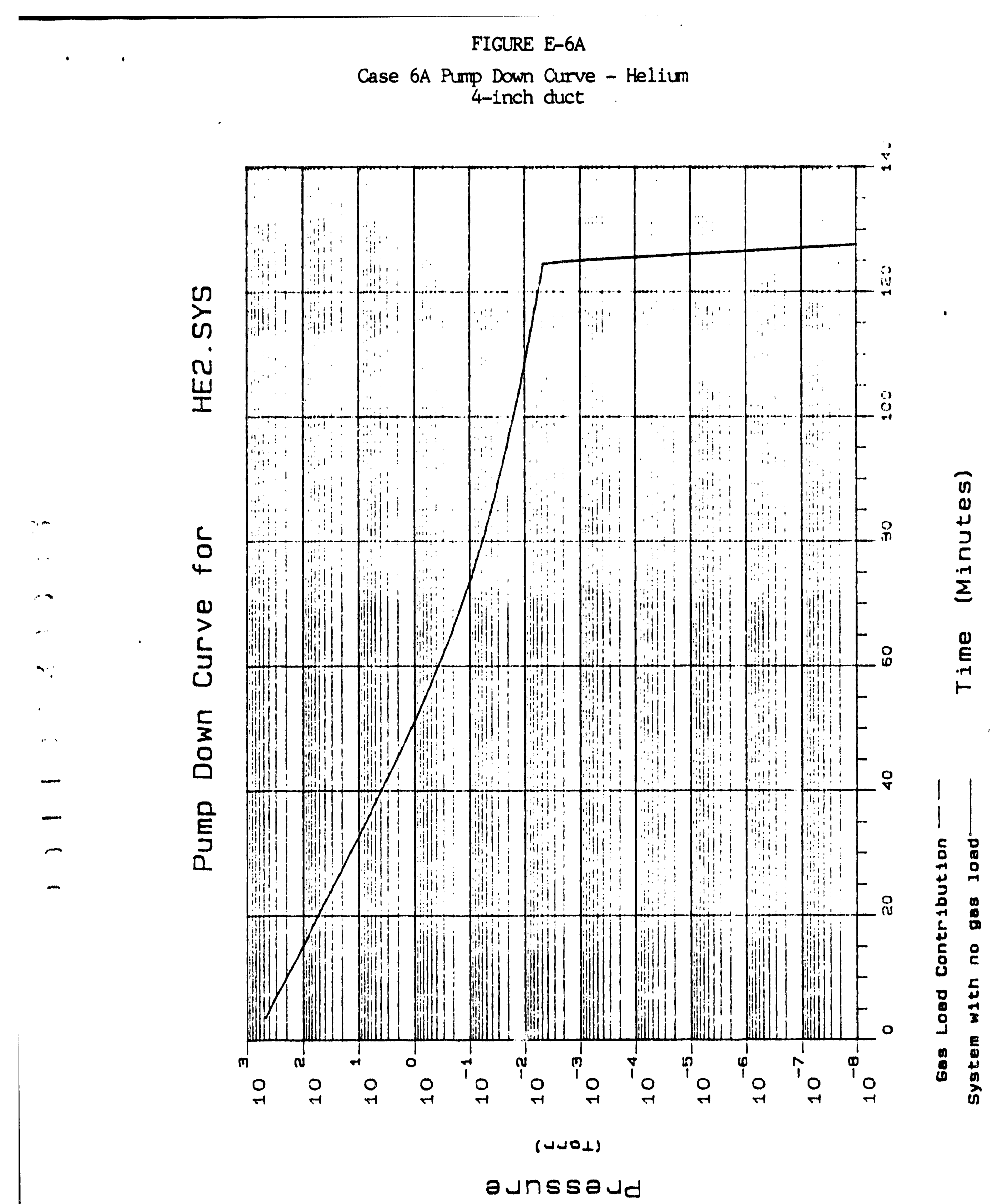


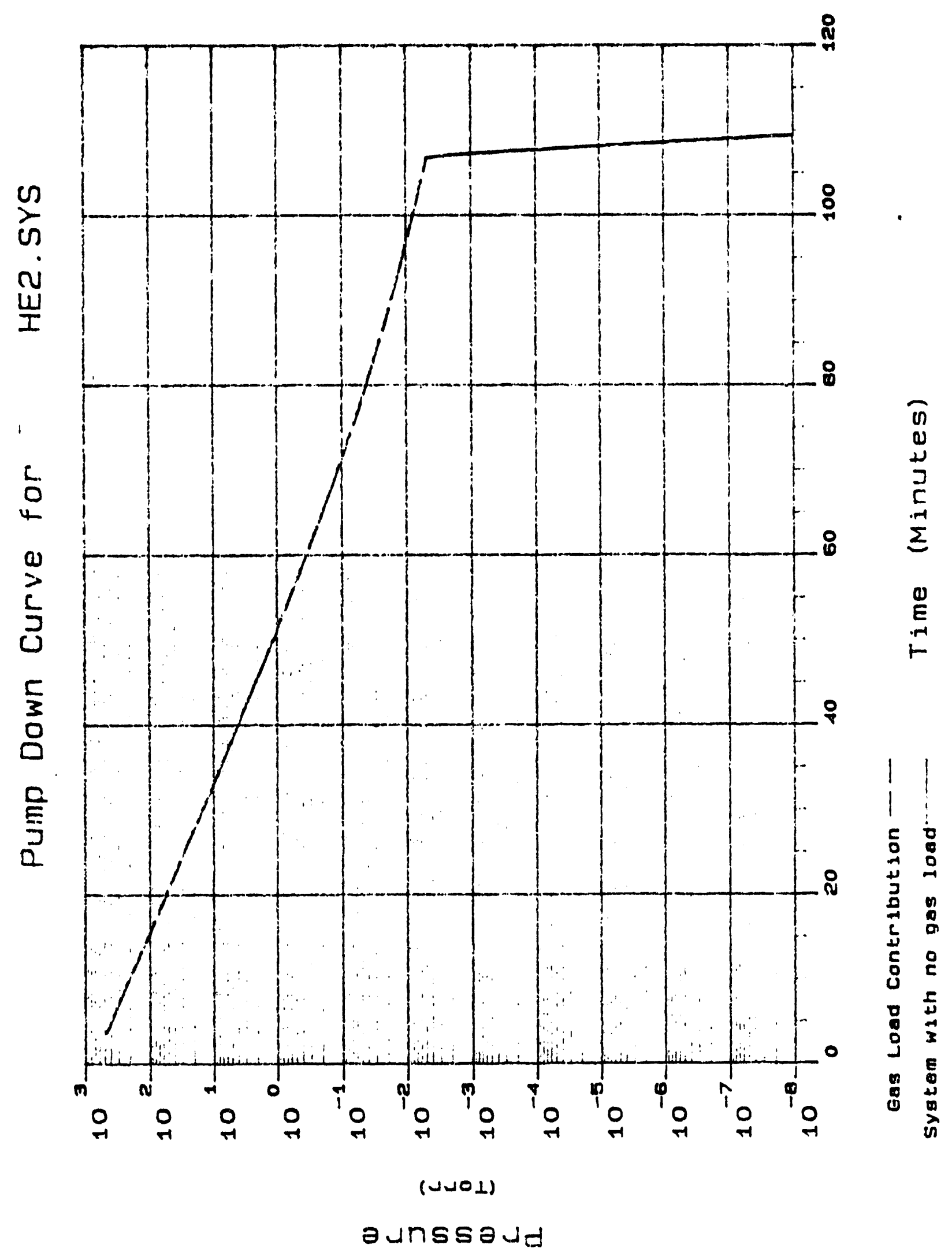




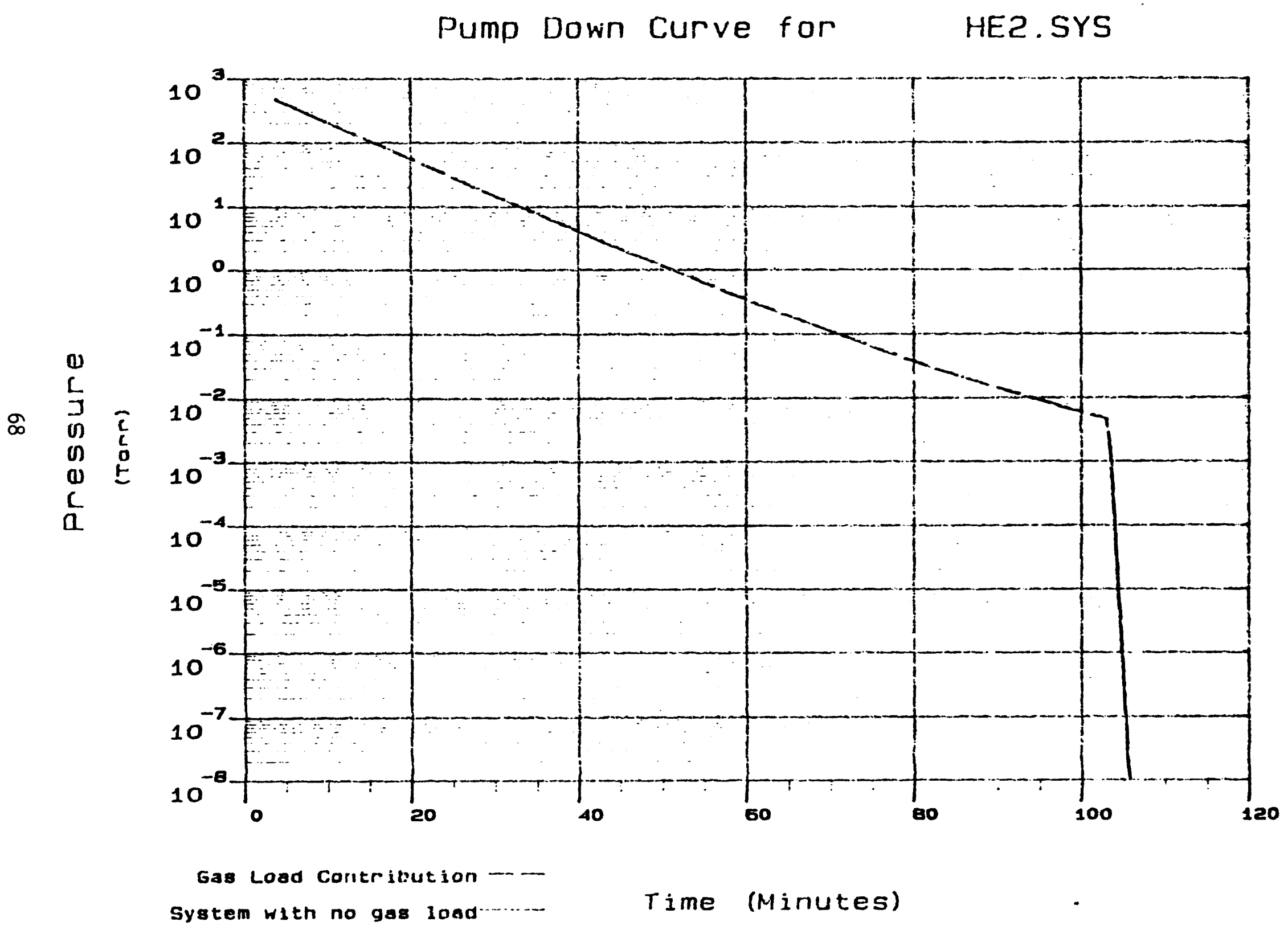


Pump Down Curve for HE4.SYS

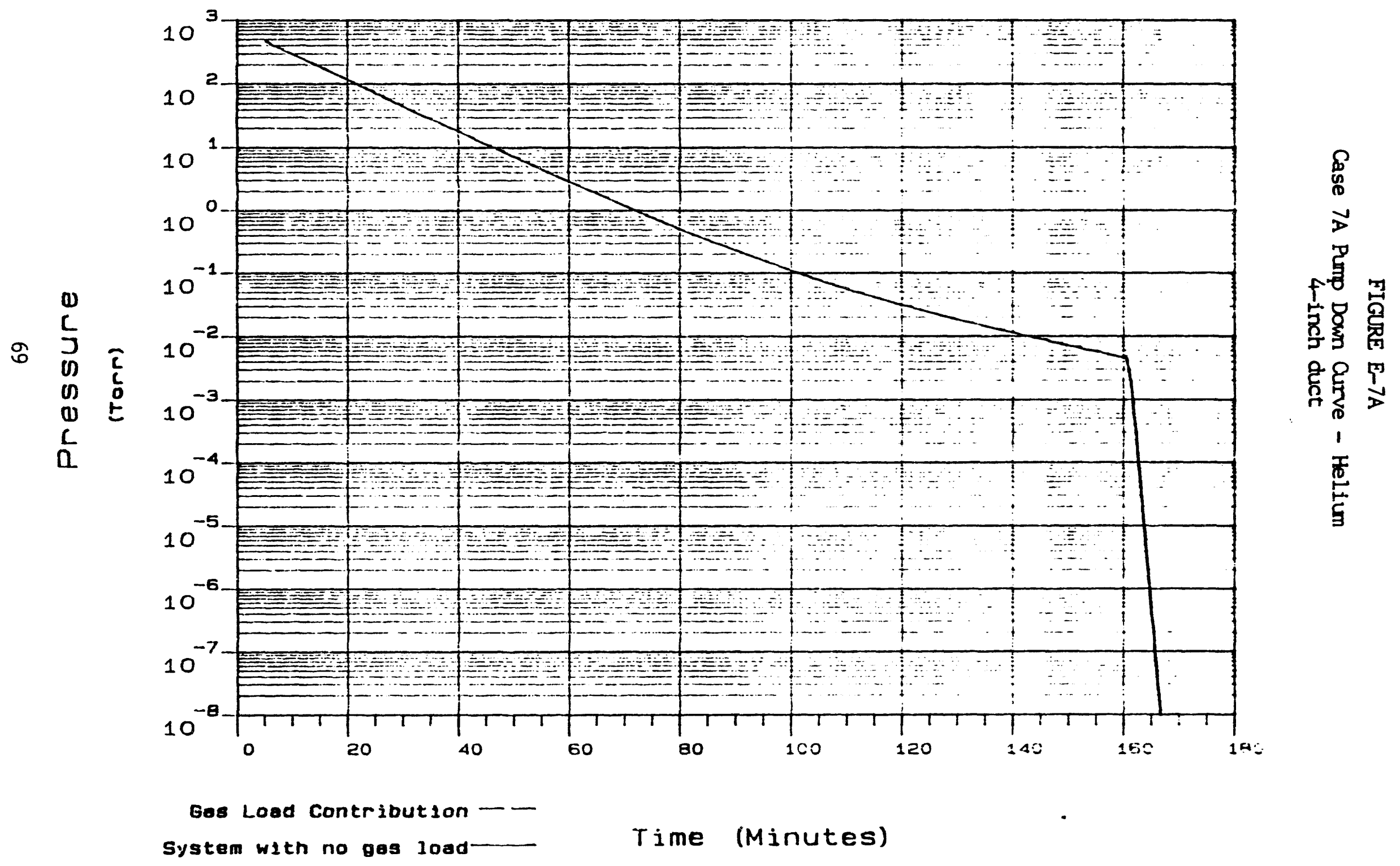


Pump Dowa Curve for

HЕ山.SYS

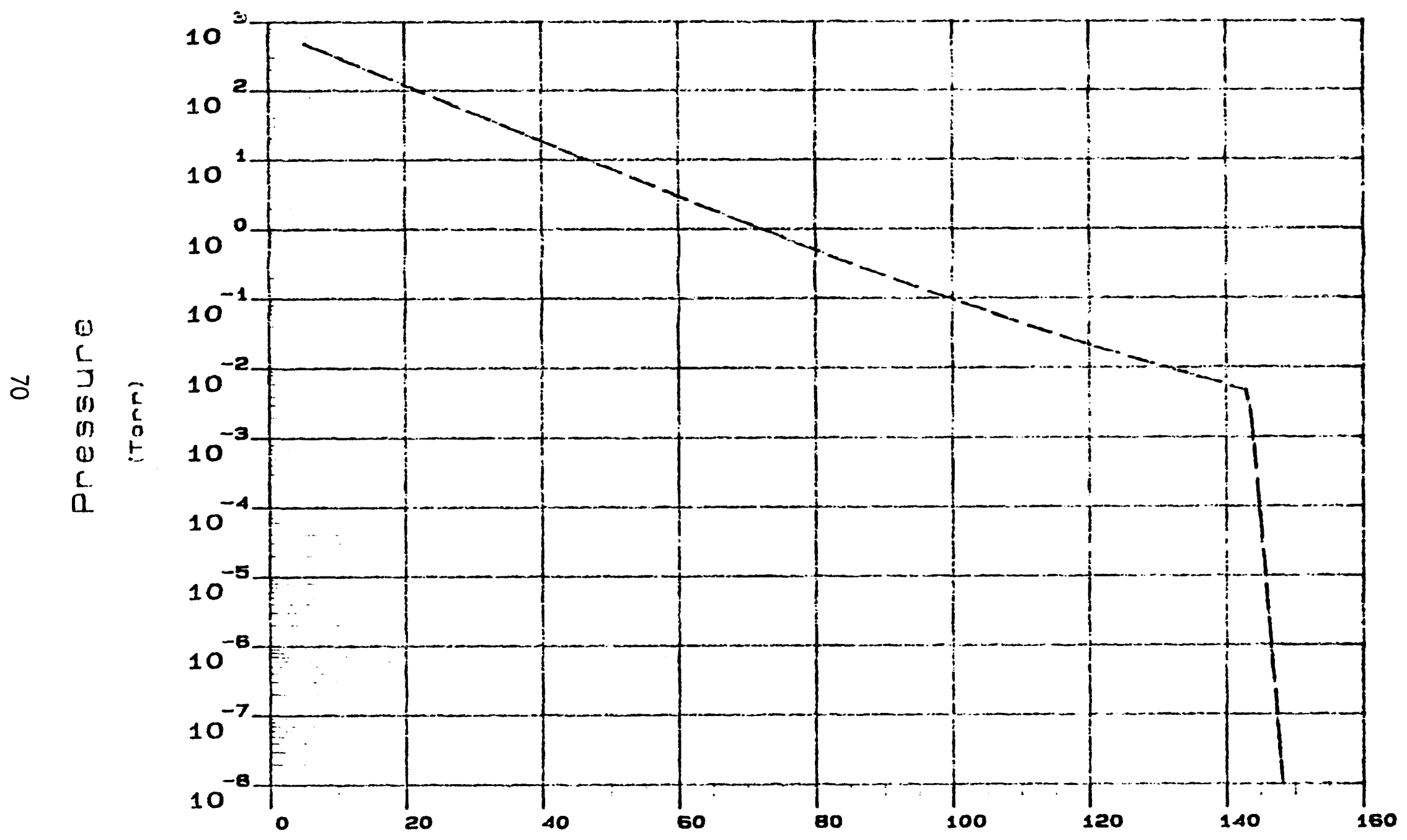

Gae Load Coritribution …system with no ous load line (Manites) 
FIGURE E-7C

Case 7C Pump Down Curve - Helium

8-inch duct

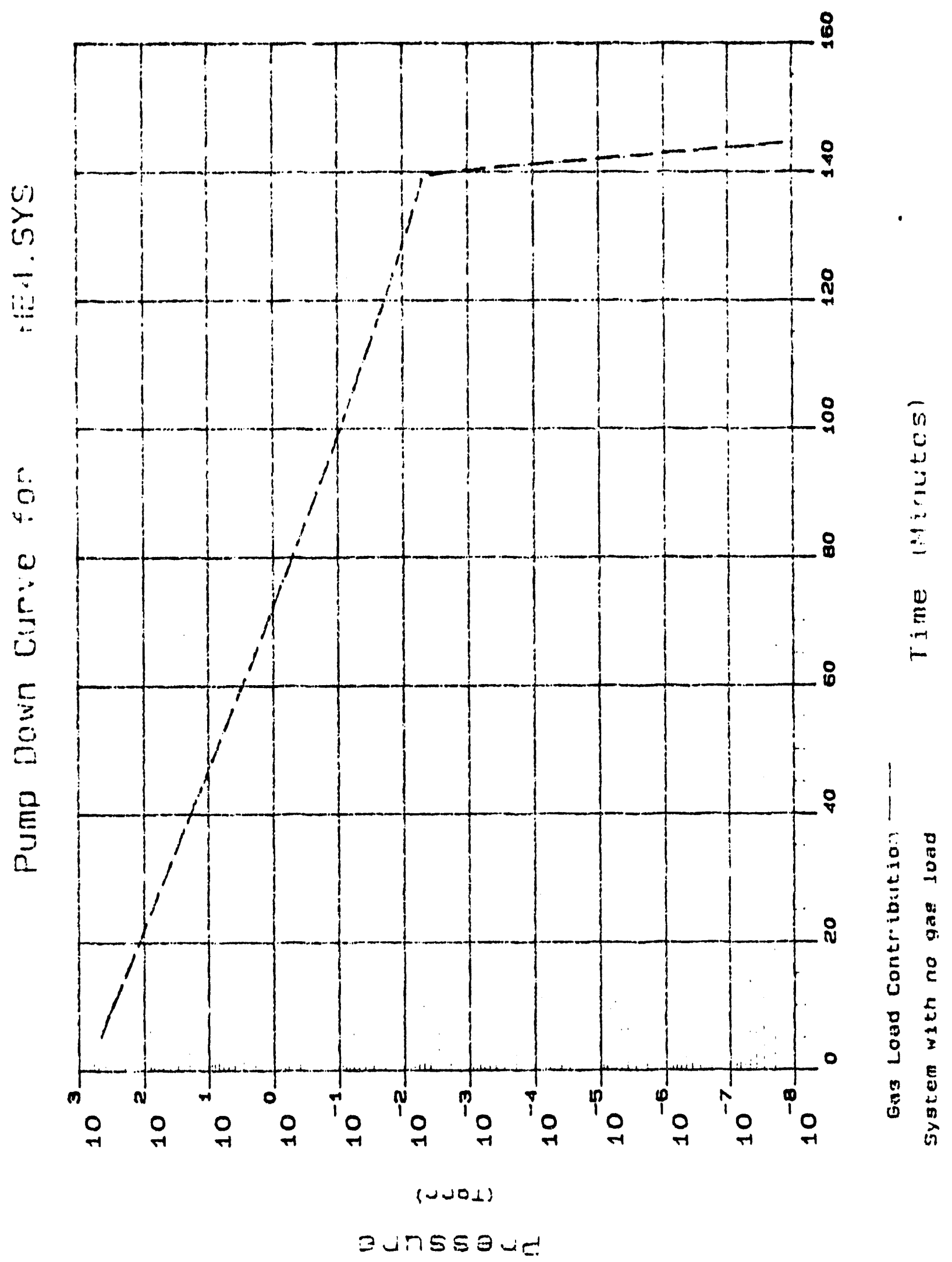




\section{Pump Down Curve for STOKES.SYS}

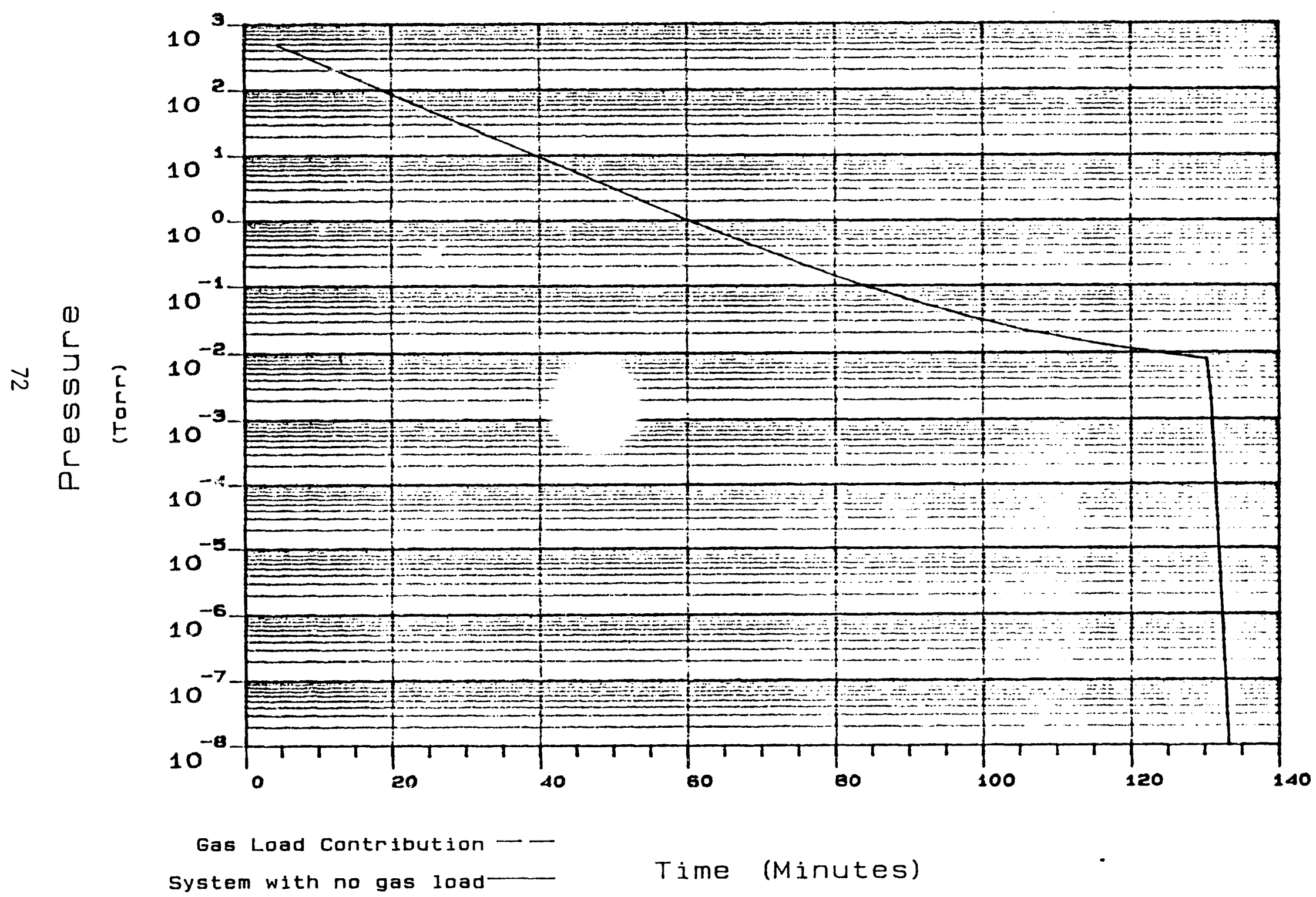




\section{Pump Down Curve for STOKES.SYS}

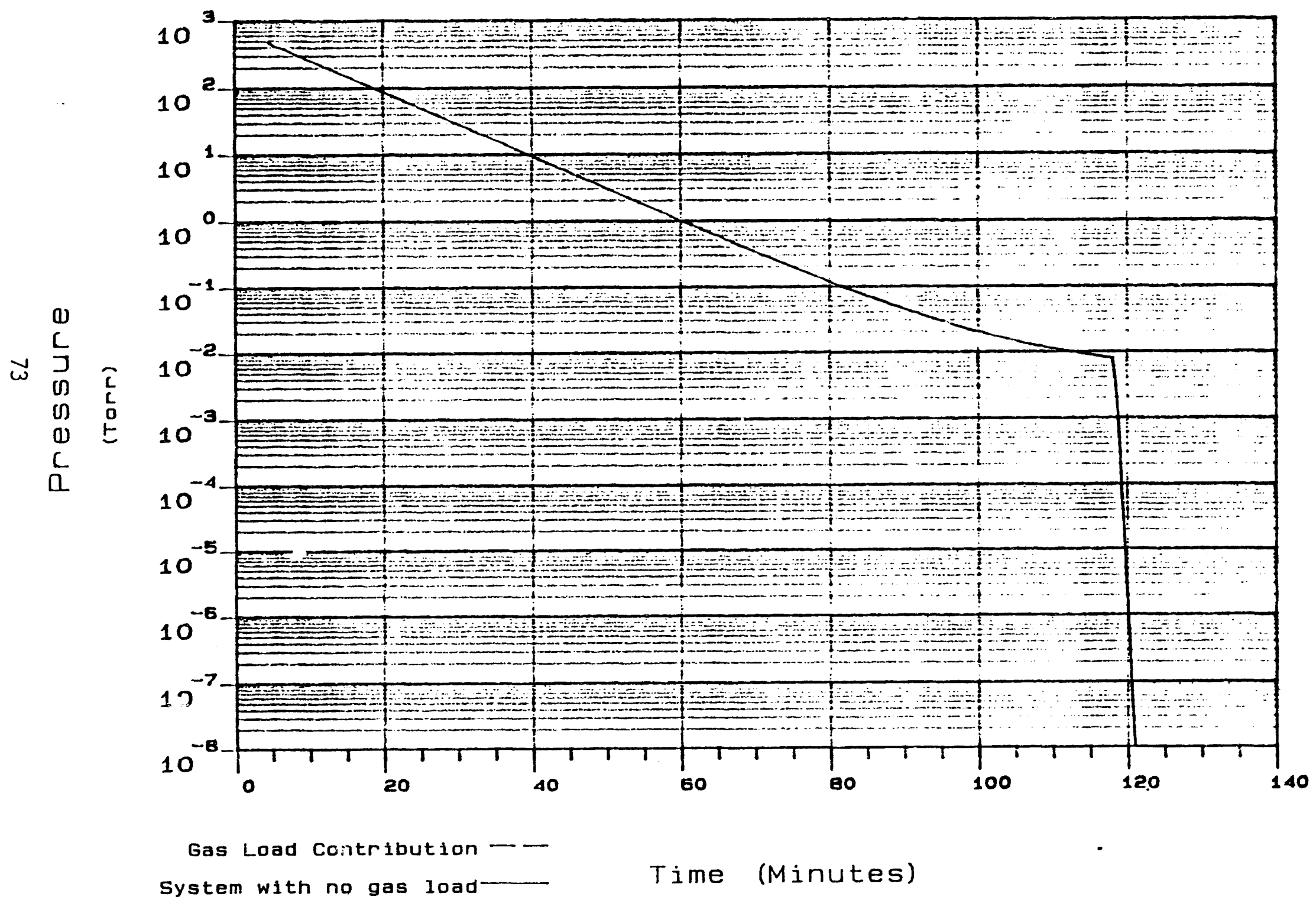




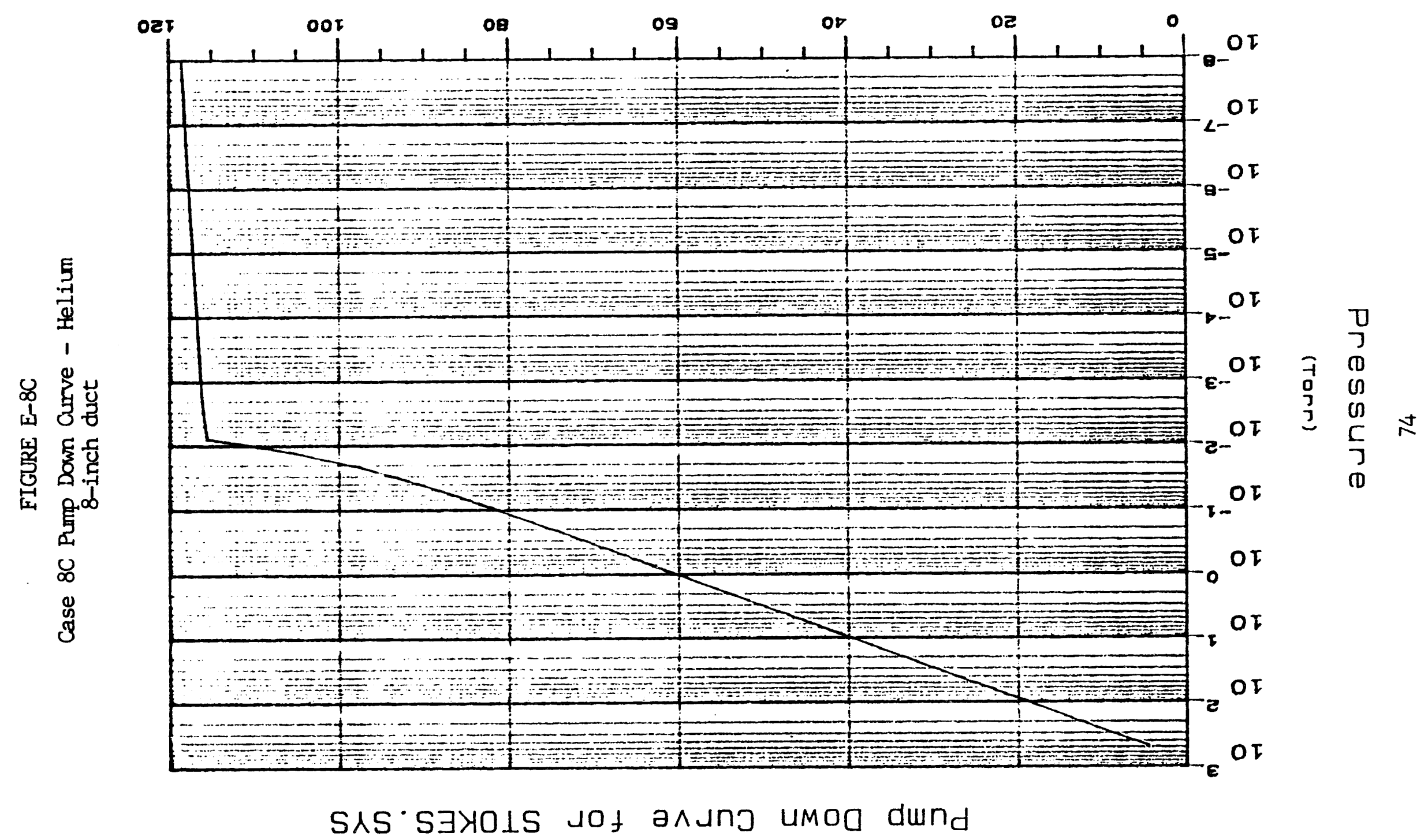

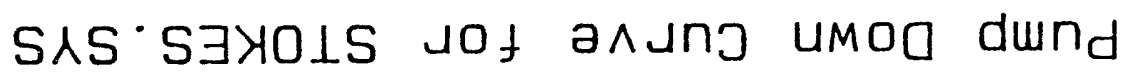


VACUUM SYSTEM DESIGN

Baseline Configration

$-$

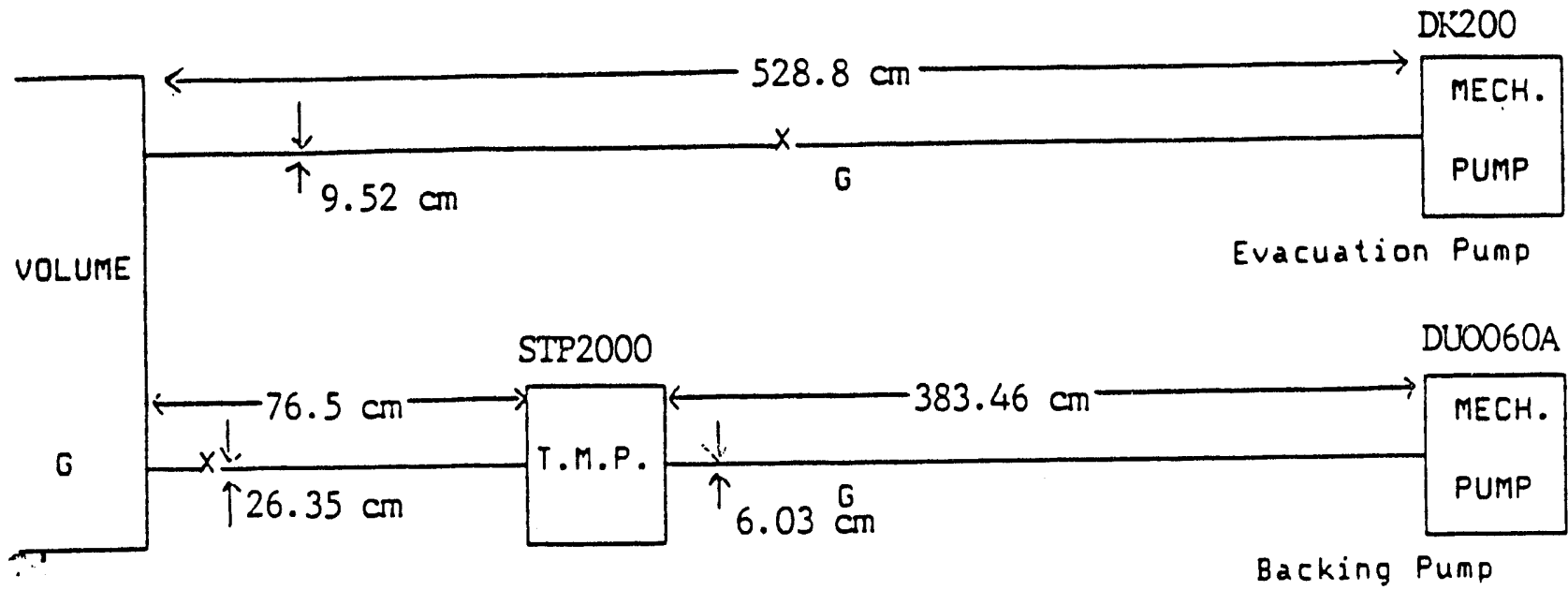

$\cdots$

$\because$

[Tab]-to Review

[Enter]-Accept Schematic

$[E s c]-B a c k u p$ 
COMMERCIAL TURBOMOLECULAR PUMP DATA

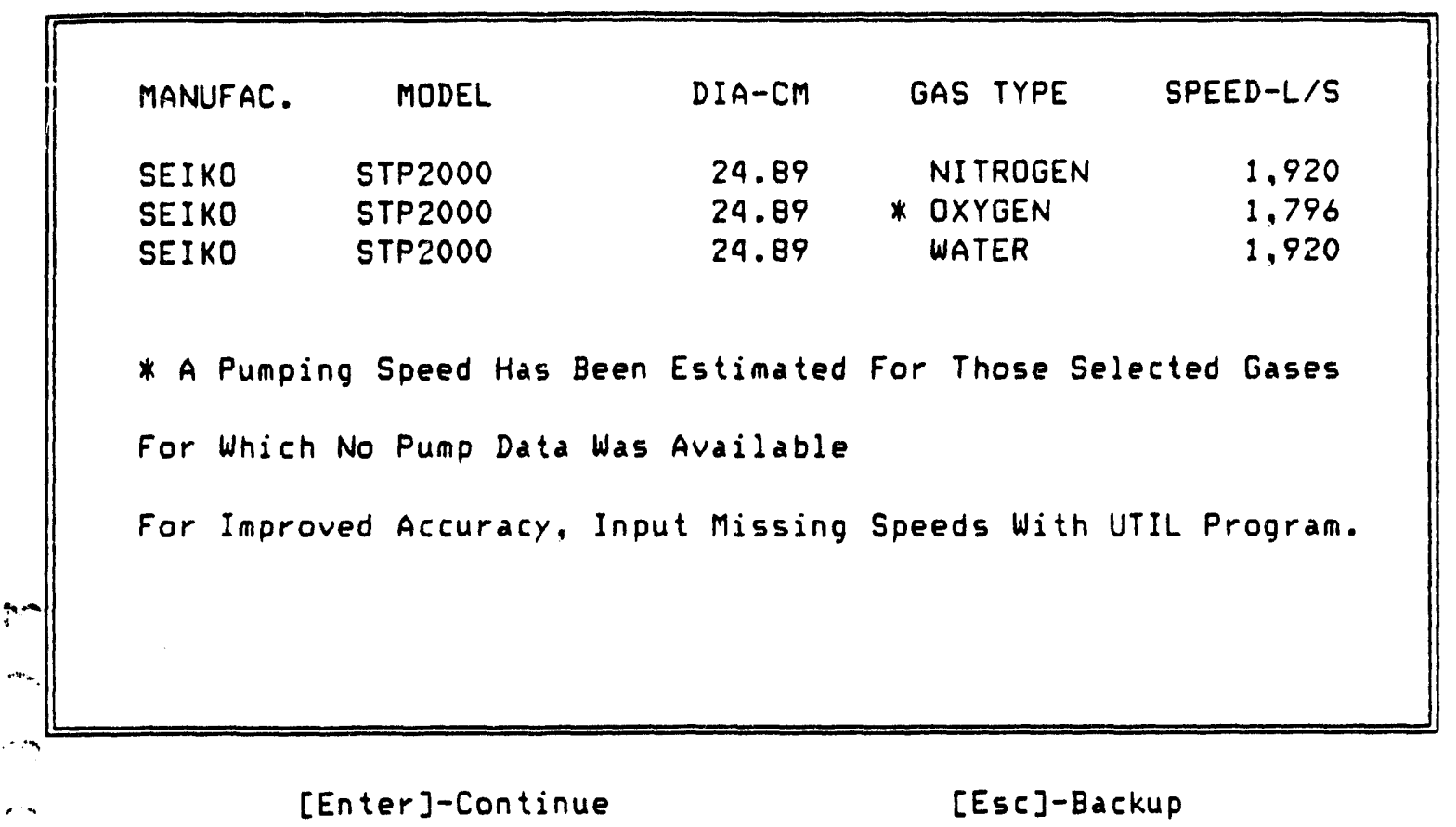

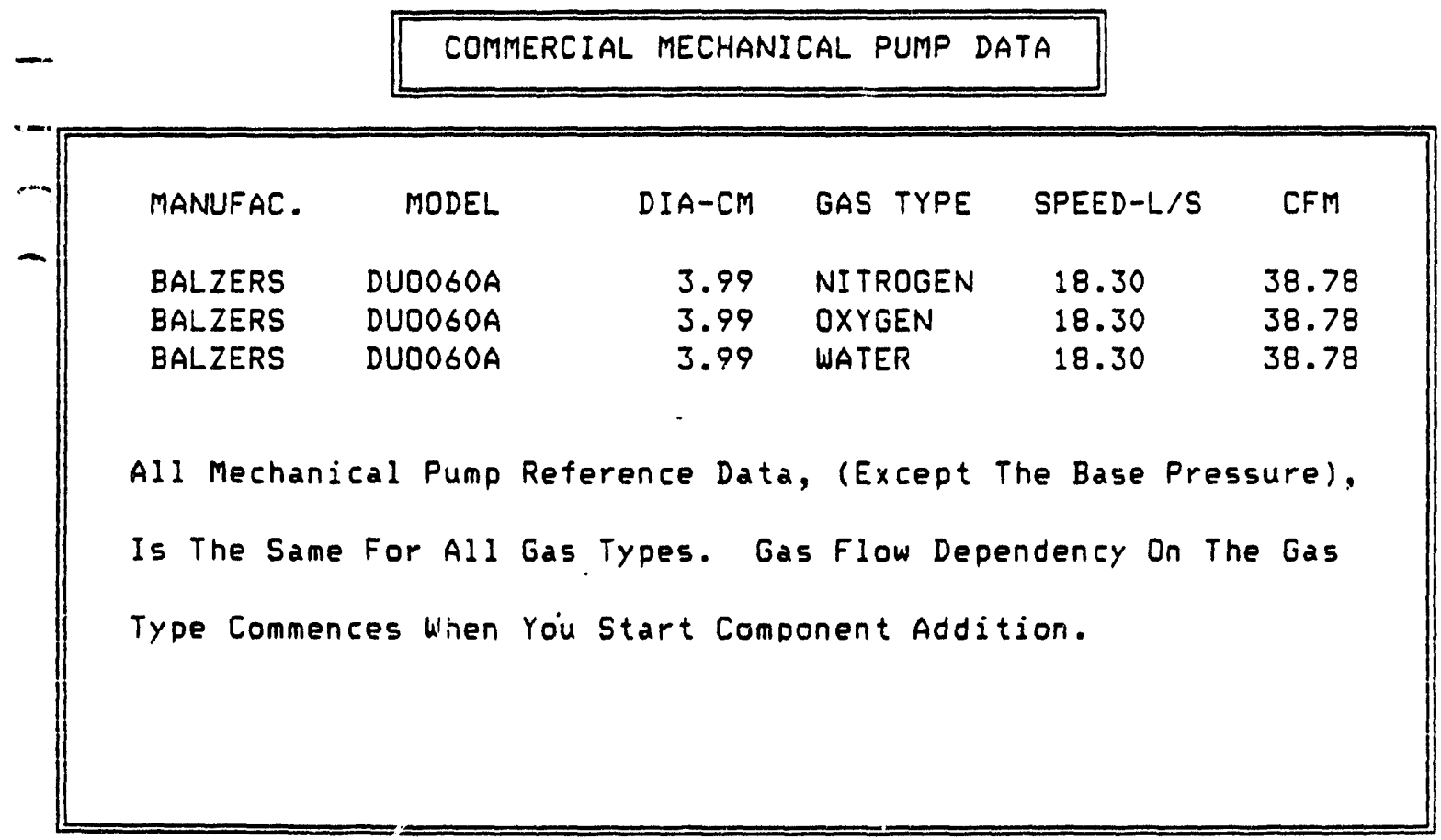

[Enter]-Continue

[Esc]-Backup 
COMMERCIAL MECHANICAL PUMP DATA

$\begin{array}{lrrlll}\text { MANUFAC. } & \text { MODEL } & \text { DIA-CM } & \text { GAS TYPE } & \text { SPEED-L/S } & \text { CFM } \\ & & & & & \\ \text { LEYBOLD } & \text { DK200 } & 7.62 & \text { NITROGEN } & 54.70 & 115.91 \\ \text { LEYBOLD } & \text { DK200 } & 7.62 & \text { OXYGEN } & 54.70 & 115.91 \\ \text { LEYBOLD } & \text { DK200 } & 7.62 & \text { WATER } & 54.70 & 115.91\end{array}$

All Mechanical Pump Reference Data, (Except The Base Pressure), Is The Same For All Gas Types. Gas Flow Dependency On The Gas Type Commences when You Start Component Addition.

[Enter]-Continue [Esc]-Backup




\begin{tabular}{|clcc|}
\hline FORELINE VAC. SYST T $=366 \mathrm{~K}$ & COMPONENT ADDITION (in CM) \\
\hline \hline NO. & DESCRIPTION & DIAMETER & LENGTH \\
1 & DUOO6OA & 3.99 & \\
2 & Circular Tube & 6.03 & 383.46 \\
3 & Tube with Bend & 6.03 & 11.99 \\
4 & Tube with Bend & 6.03 & 23.94 \\
5 & Straight thru Valve & 6.03 & 14.99 \\
6 & Gage Location & 6.03 & \\
7 & System Mult. $=2$ & 8.53 & \\
8 & Line Terminus & & \\
9 & & & \\
10 & & & \\
11 & & & \\
12 & & \\
13 & & \\
14 & & \\
15 & & \\
16 & & \\
17 & & \\
18 & & & \\
\hline
\end{tabular}

Press Any Key To Continue 


\begin{tabular}{|cc|cc|}
\hline HIGH VACUUM SYSTEM T $=366 \mathrm{~K}$ & COMPONENT ADDITION (in CM) \\
\hline No. & DESCRIPTION & DIAMETER & LENGTH \\
1 & STP2000 & 24.89 & \\
2 & Circular Tube & 26.35 & 76.50 \\
3 & Straight thru Valve & 26.35 & 14.00 \\
4 & Gage Location & 26.35 & \\
5 & System Mult. $=2$ & 37.26 & \\
6 & Line Terminus & & \\
7 & & & \\
8 & & & \\
9 & & & \\
10 & & \\
11 & & \\
12 & & \\
13 & & \\
14 & & \\
15 & & \\
16 & & \\
17 & & \\
18 & & \\
\hline
\end{tabular}

Press Any Key To Continue 


\begin{tabular}{|cc|cc|}
\hline EVACUATION SYSTEM & T= 366 K & COMPONENT ADDITION (in CM) \\
\hline No. & DESCRIPTION & DIAMETER & LENGiH \\
1 & DK200 & 7.62 & \\
2 & Circular Tube & 9.52 & 528.80 \\
3 & Tube with Bend & 9.52 & 23.94 \\
4 & Tube with Bend & 9.52 & 23.94 \\
5 & Tube with Bend & 9.52 & 14.63 \\
6 & Gage Location & 9.52 & \\
7 & Line Terminus & & \\
8 & & & \\
9 & & & \\
10 & & & \\
11 & & & \\
12 & & \\
13 & & & \\
14 & & \\
15 & & \\
16 & & \\
17 & & \\
18 & & & \\
\hline
\end{tabular}

Press Any Key To Continue 


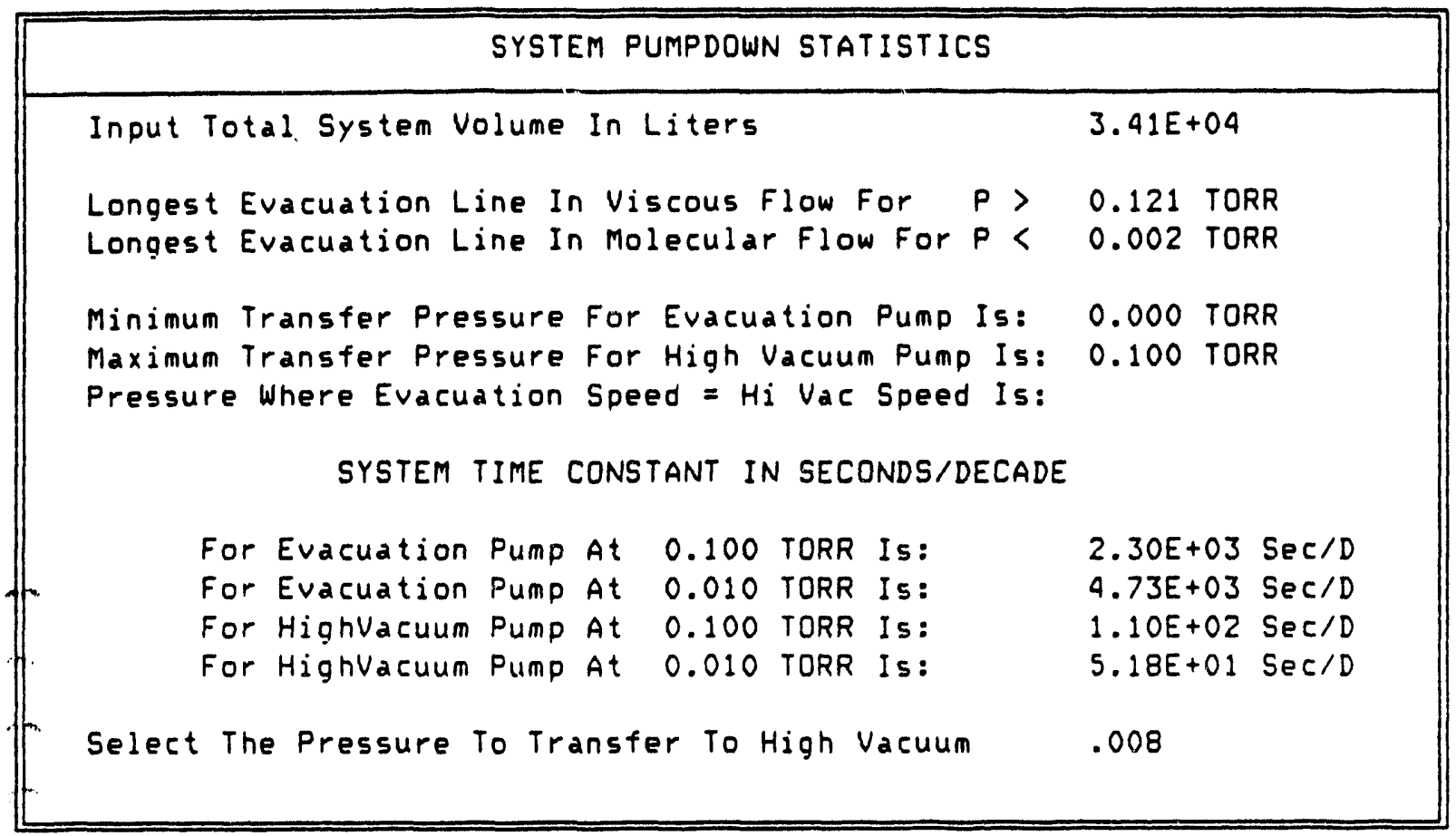



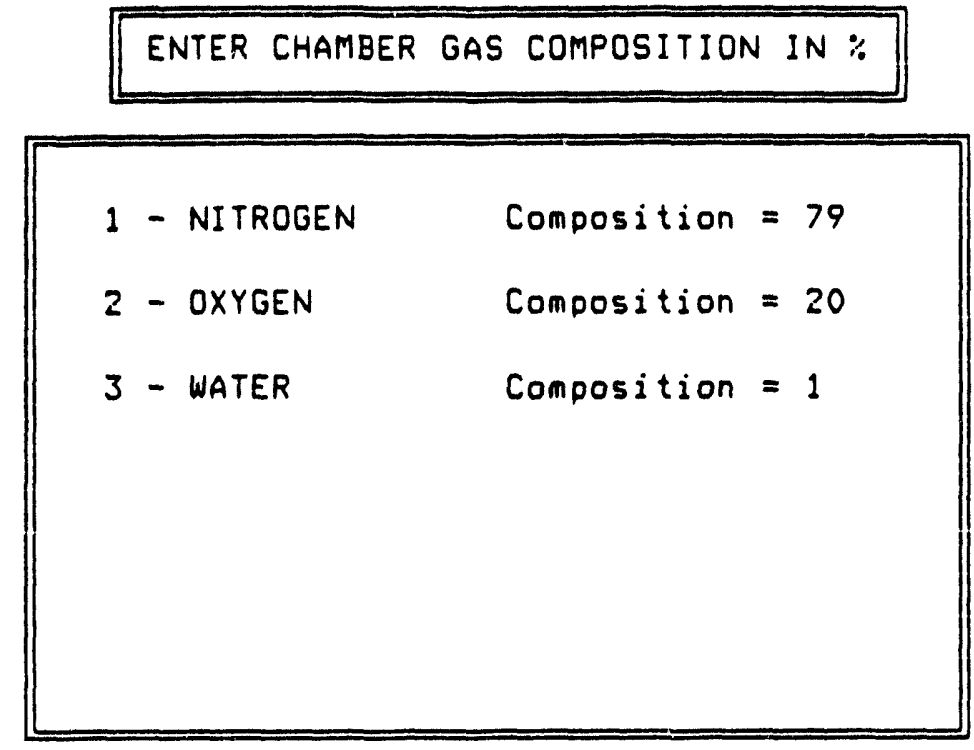


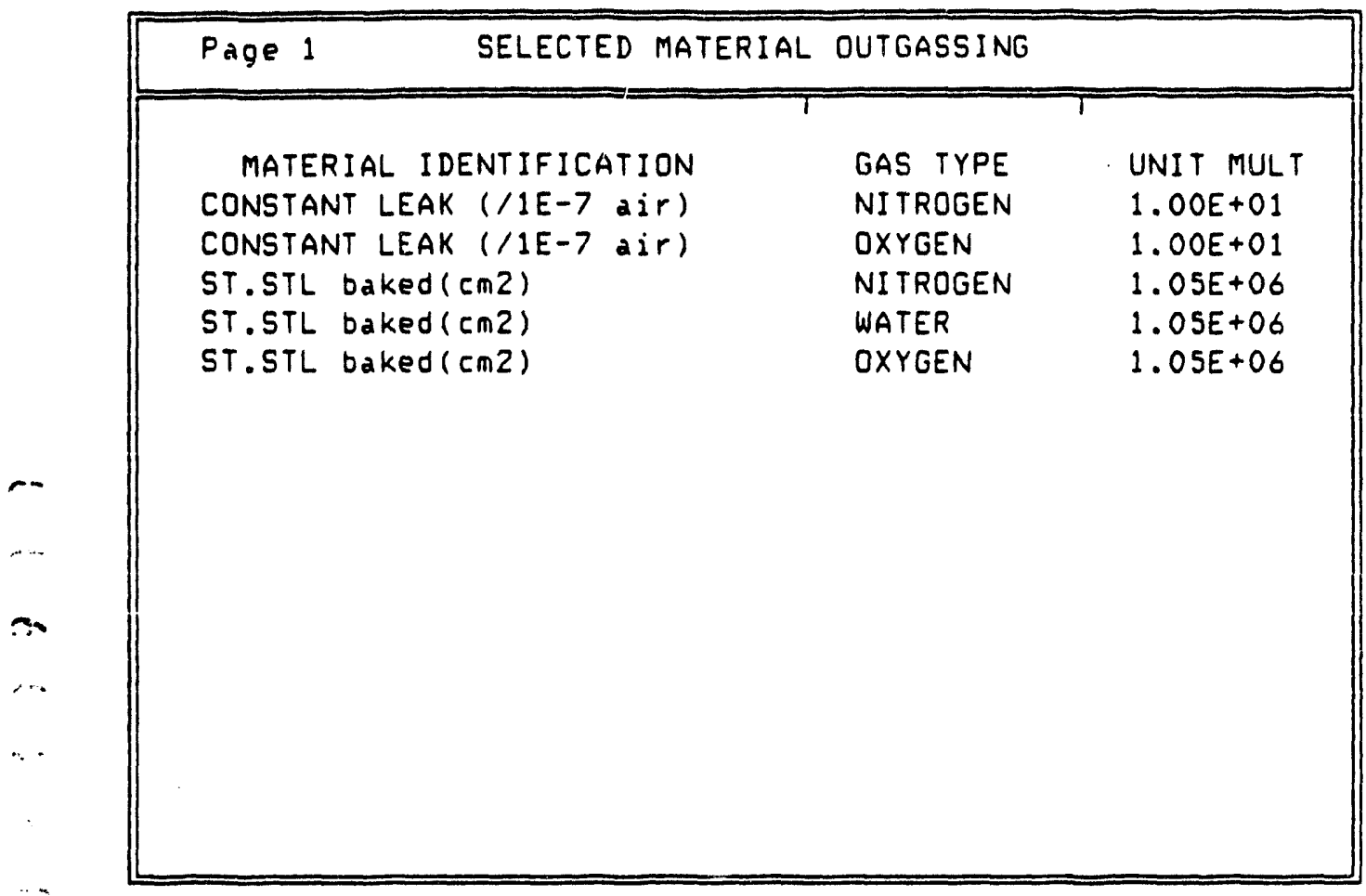

[Enter]-Next Page 


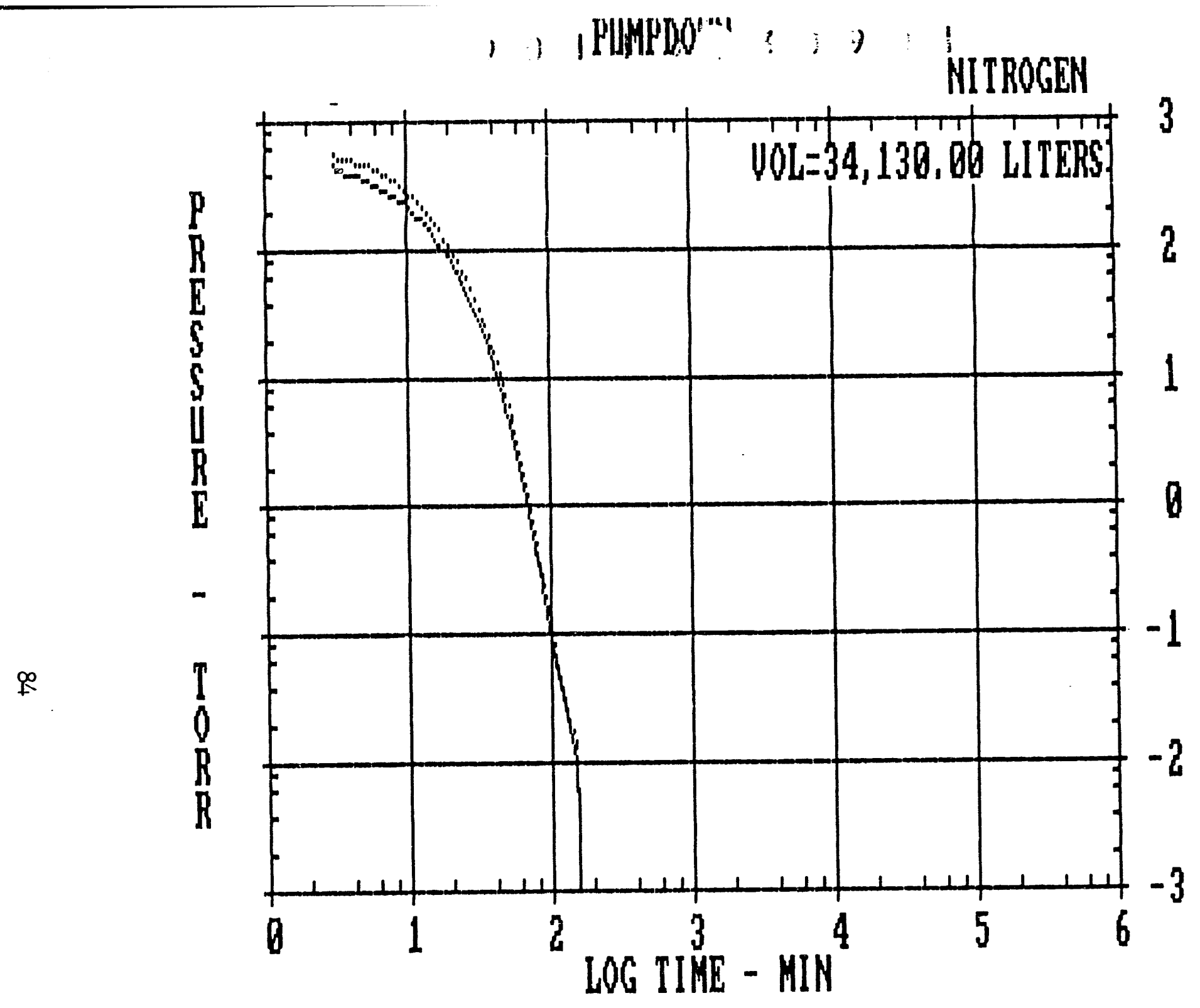

F1-Shift F2-Print F3-Read F4-Data F5-Cas [Enter]-Continue 


\begin{tabular}{|r|c|c|c|c|c|}
\hline No. & TIME & PUMPDOWN P & OUTGAS P & TOTAL PRES & OUTGASSING \\
\hline & MINUTES & TORR & TORR & TORR & $. T-L / S$ \\
\hline 1 & $3.08 E+00$ & $4.47 E+02$ & $0.00 E+00$ & $4.47 E+02$ & \\
2 & $3.56 E+00$ & $4.22 E+02$ & $0.00 E+00$ & $4.22 E+02$ & \\
3 & $4.11 E+00$ & $3.99 E+02$ & $0.00 E+00$ & $3.99 E+02$ & \\
4 & $4.74 E+00$ & $3.77 E+02$ & $0.00 E+00$ & $3.77 E+02$ & \\
5 & $5.48 E+00$ & $3.55 E+02$ & $0.00 E+00$ & $3.55 E+02$ & \\
6 & $6.33 E+00$ & $3.25 E+02$ & $0.00 E+00$ & $3.25 E+02$ & \\
7 & $7.30 E+00$ & $2.98 E+02$ & $0.00 E+00$ & $2.98 E+02$ & \\
8 & $8.44 E+00$ & $2.67 E+02$ & $0.00 E+00$ & $2.67 E+02$ & \\
9 & $9.74 E+00$ & $2.37 E+02$ & $0.00 E+00$ & $2.37 E+02$ & \\
10 & $1.12 E+01$ & $2.04 E+02$ & $0.00 E+00$ & $2.04 E+02$ & \\
11 & $1.30 E+01$ & $1.73 E+02$ & $0.00 E+00$ & $1.73 E+02$ & \\
12 & $1.50 E+01$ & $1.43 E+02$ & $0.00 E+00$ & $1.43 E+02$ & \\
13 & $1.73 E+01$ & $1.15 E+02$ & $0.00 E+00$ & $1.15 E+02$ & \\
14 & $2.00 E+01$ & $8.93 E+01$ & $0.00 E+00$ & $8.93 E+01$ & \\
15 & $2.31 E+01$ & $6.68 E+01$ & $0.00 E+00$ & $6.68 E+01$ & \\
16 & $2.67 E+01$ & $4.77 E+01$ & $0.00 E+00$ & $4.77 E+01$ & \\
17 & $3.08 E+01$ & $3.24 E+01$ & $0.00 E+00$ & $3.24 E+01$ & \\
& & & & & \\
\hline
\end{tabular}

[Enter]-Next Page

[Esc]-Exit

Page 2

PUMPDOWN

NITROGEN

\begin{tabular}{|c|c|c|c|c|c||}
\hline No. & TIME & PUMPDOWN P & OUTGAS P & TOTAL PRES & OUTGASSING \\
\hline & MINUTES & TORR & TORR & TORR & $. T-L / S$ \\
\hline 18 & $3.56 E+01$ & $2.07 E+01$ & $0.00 E+00$ & $2.07 E+01$ & \\
19 & $4.11 E+01$ & $1.24 E+01$ & $0.00 E+00$ & $1.24 E+01$ & \\
20 & $4.74 E+01$ & $6.92 E+00$ & $0.00 E+00$ & $6.92 E+00$ & \\
21 & $5.48 E+01$ & $3.54 E+00$ & $0.00 E+00$ & $3.54 E+00$ & \\
22 & $6.33 E+01$ & $1.65 E+00$ & $0.00 E+00$ & $1.65 E+00$ & \\
23 & $7.30 E+01$ & $7.08 E-01$ & $0.00 E+00$ & $7.08 E-01$ & \\
24 & $8.44 E+01$ & $2.83 E-01$ & $0.00 E+00$ & $2.83 E-01$ & \\
25 & $9.74 E+01$ & $1.12 E-01$ & $0.00 E+00$ & $1.12 E-01$ & \\
26 & $1.12 E+02$ & $4.64 E-02$ & $0.00 E+00$ & $4.64 E-02$ & \\
27 & $1.30 E+02$ & $2.11 E-02$ & $0.00 E+00$ & $2.11 E-02$ & \\
28 & $1.50 E+02$ & $1.04 E-02$ & $0.00 E+00$ & $1.04 E-02$ & \\
29 & $1.71 E+02$ & $2.23 E-08$ & $0.00 E+00$ & $2.23 E-08$ & \\
30 & $2.00 E+02$ & $0.00 E+00$ & $0.00 E+00$ & $0.00 E+00$ & \\
31 & $2.31 E+02$ & $0.00 E+00$ & $0.00 E+00$ & $0.00 E+00$ & \\
32 & $2.67 E+02$ & $0.00 E+00$ & $0.00 E+00$ & $0.00 E+00$ & \\
33 & $3.08 E+02$ & $0.00 E+00$ & $0.00 E+00$ & $0.00 E+00$ & \\
34 & $3.56 E+02$ & $0.00 E+00$ & $0.00 E+00$ & $0.00 E+00$ & \\
\hline
\end{tabular}

[Enter]-Next Page

$[E s c]-E x i t$ 


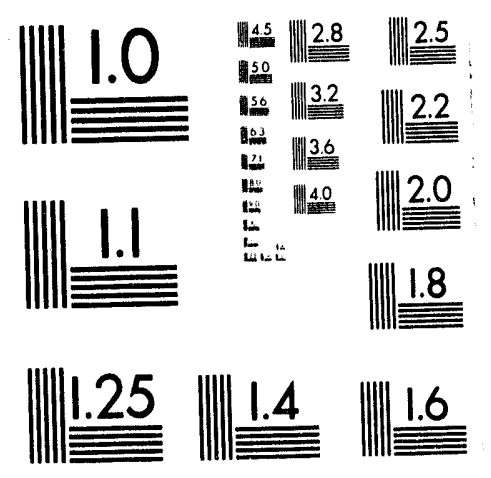


$\mid$
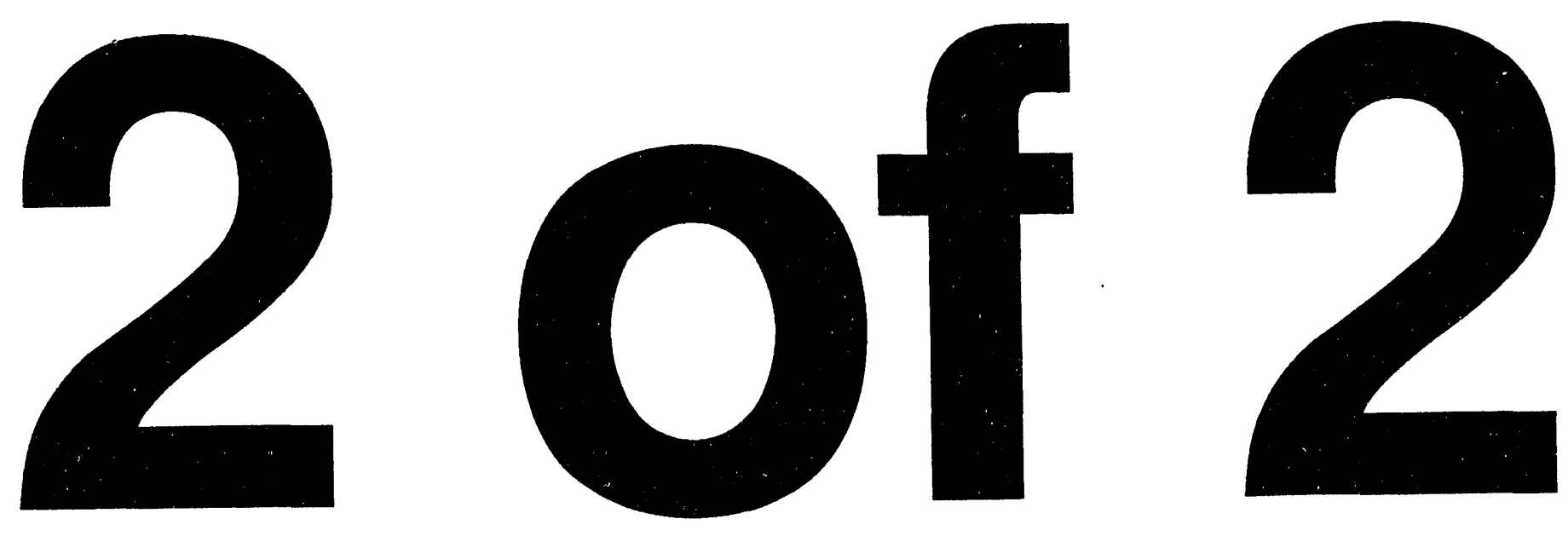


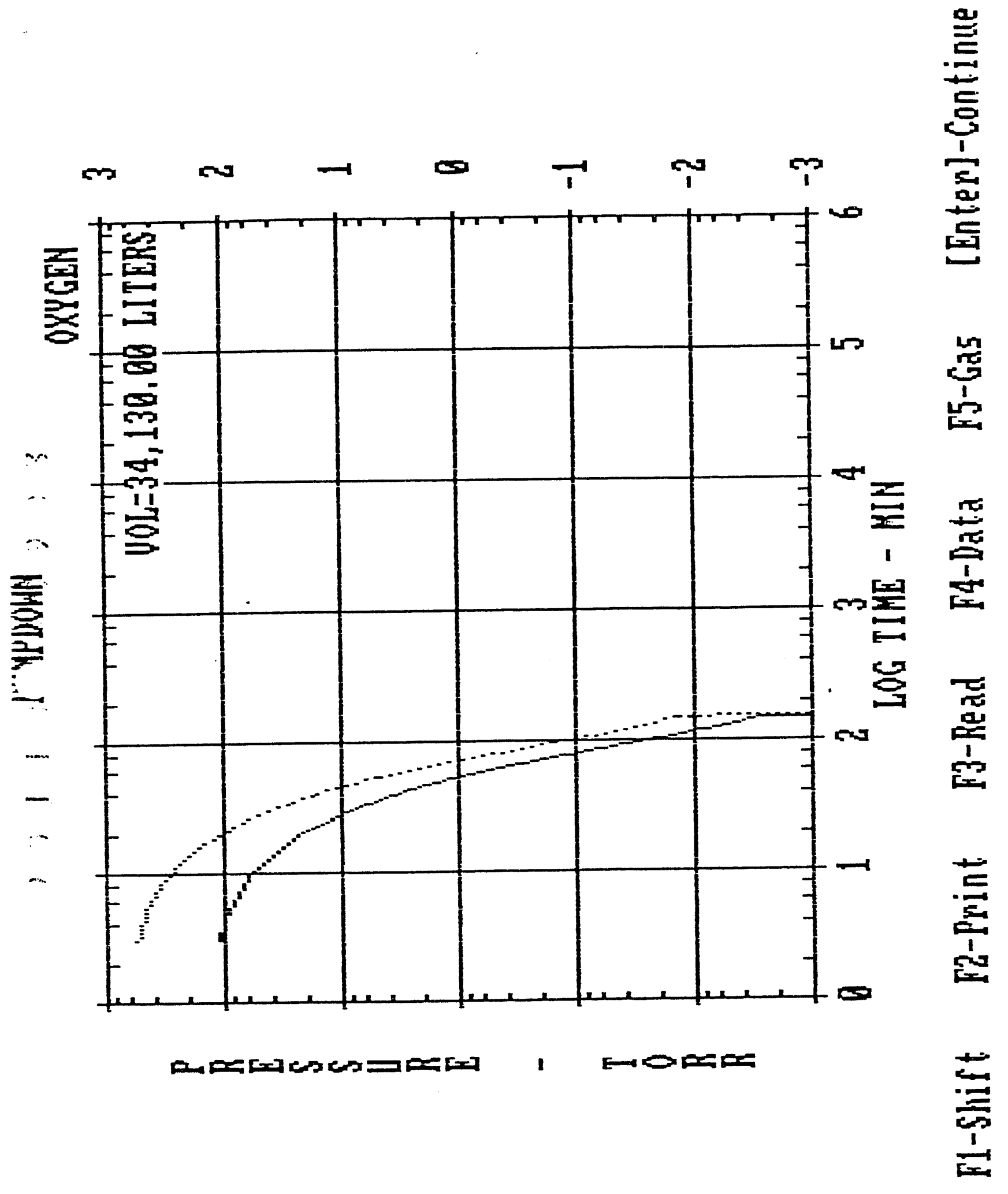


Page 1

PUMPDOWN

OXYGEN

\begin{tabular}{|c|c|c|c|c|c|}
\hline No. & TIME & PUMPDOWN P & OUTGAS P & TOTAL PRES & OUTGASSING \\
\hline & MINUTES & TORR & TORR & TORR & $. T-L / S$ \\
\hline 1 & $3.08 E+00$ & $1.13 E+02$ & $0.00 E+00$ & $1.13 E+02$ & \\
\hline 2 & $3.56 E+00$ & $1.07 E+02$ & $0.00 E+00$ & $1.07 E+02$ & \\
\hline 3 & $4.11 E+00$ & $1.01 E+02$ & $0.00 E+00$ & $1.01 E+02$ & \\
\hline 4 & $4.74 E+00$ & $9.56 E+01$ & $0.00 E+00$ & $9.56 E+01$ & \\
\hline 5 & $5.48 E+00$ & $8.98 E+01$ & $0.00 E+00$ & $8.98 E+01$ & \\
\hline 6 & $6.33 E+00$ & $8.23 E+01$ & $0.00 E+00$ & $8.23 E+01$ & \\
\hline 7 & $7.30 E+00$ & $7.53 E+01$ & $0.00 E+00$ & $7.53 E+01$ & \\
\hline 8 & $8.44 E+00$ & $6.75 E+01$ & $0.00 E+00$ & $6.75 E+01$ & \\
\hline 9 & $9.74 E+00$ & $5.99 E+01$ & $0.00 E+00$ & $5.99 E+01$ & \\
\hline 10 & $1.12 E+01$ & $5.17 E+01$ & $0.00 E+00$ & $5.17 E+01$ & \\
\hline 11 & $1.30 E+01$ & $4.39 E+01$ & $0.00 E+00$ & $4.39 E+01$ & \\
\hline 12 & 1. $50 E+01$ & $3.63 E+01$ & $0.00 E+00$ & $3.63 E+01$ & \\
\hline 13 & $1.73 E+01$ & $2.91 E+01$ & $0.00 E+00$ & $2.91 E+01$ & \\
\hline 14 & $2.00 E+01$ & $2.26 E+01$ & $0.00 E+00$ & $2.26 E+01$ & \\
\hline 15 & $2.31 E+01$ & $1.69 E+01$ & $0.00 E+00$ & 1. $69 E+01$ & \\
\hline 16 & $2.67 E+01$ & $1.21 E+01$ & $0.00 E+00$ & $1.21 E+01$ & \\
\hline 17 & $3.08 E+01$ & $8.19 E+00$ & $0.00 E+00$ & $8.19 E+00$ & \\
\hline
\end{tabular}

[Enter]-Next Page

[Esc]-Exit

Page 2

PUMPDOWN

OXYGEN

\begin{tabular}{|c|c|c|c|c|c|}
\hline NO. & TIME & PUMPDOWN P & OUTGAS P & TOTAL PRES & OUTGASSING \\
\hline & MINUTES & TORR & TORR & TORR & $. T-L / S$ \\
\hline 18 & $3.56 E+01$ & $5.25 E+00$ & $0.00 E+00$ & $5.25 E+00$ & \\
19 & $4.11 E+01$ & $3.15 E+00$ & $0.00 E+00$ & $3.15 E+00$ & \\
20 & $4.74 E+01$ & $1.75 E+00$ & $0.00 E+00$ & $1.75 E+00$ & \\
21 & $5.48 E+01$ & $8.95 E-01$ & $0.00 E+00$ & $8.95 E-01$ & \\
22 & $6.33 E+01$ & $4.19 E-01$ & $0.00 E+00$ & $4.19 E-01$ & \\
23 & $7.30 E+01$ & $1.79 E-01$ & $0.00 E+00$ & $1.79 E-01$ & \\
24 & $8.44 E+01$ & $7.18 E-02$ & $0.00 E+00$ & $7.18 E-02$ & \\
25 & $9.74 E+01$ & $2.83 E-02$ & $0.00 E+00$ & $2.83 E-02$ & \\
26 & $1.12 E+02$ & $1.18 E-02$ & $0.00 E+00$ & $1.18 E-02$ & \\
27 & $1.30 E+02$ & $5.35 E-03$ & $0.00 E+00$ & $5.35 E-03$ & \\
28 & $1.50 E+02$ & $2.64 E-03$ & $0.00 E+00$ & $2.64 E-03$ & \\
29 & $1.71 E+02$ & $5.65 E-09$ & $0.00 E+00$ & $5.65 E-09$ & \\
30 & $2.00 E+02$ & $0.00 E+00$ & $0.00 E+00$ & $0.00 E+00$ & \\
31 & $2.31 E+02$ & $0.00 E+00$ & $0.00 E+00$ & $0.00 E+00$ & \\
32 & $2.67 E+02$ & $0.00 E+00$ & $0.00 E+00$ & $0.00 E+00$ & \\
33 & $3.08 E+02$ & $0.00 E+00$ & $0.00 E+00$ & $0.00 E+00$ & \\
34 & $3.56 E+02$ & $0.00 E+00$ & $0.00 E+00$ & $0.00 E+00$ & \\
\hline
\end{tabular}

[Enter]-Next Page

$[E s[]-E x i t$ 


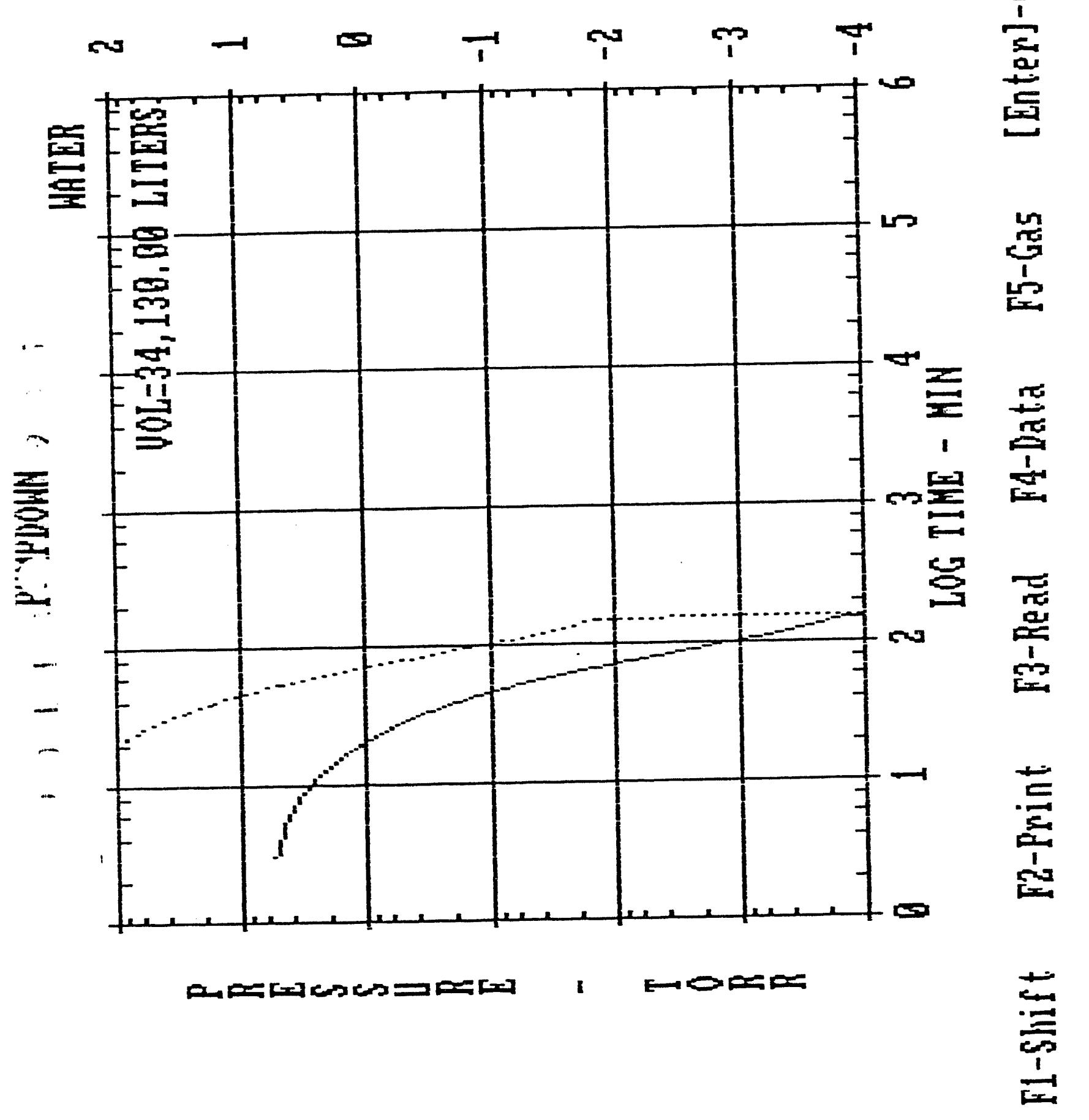




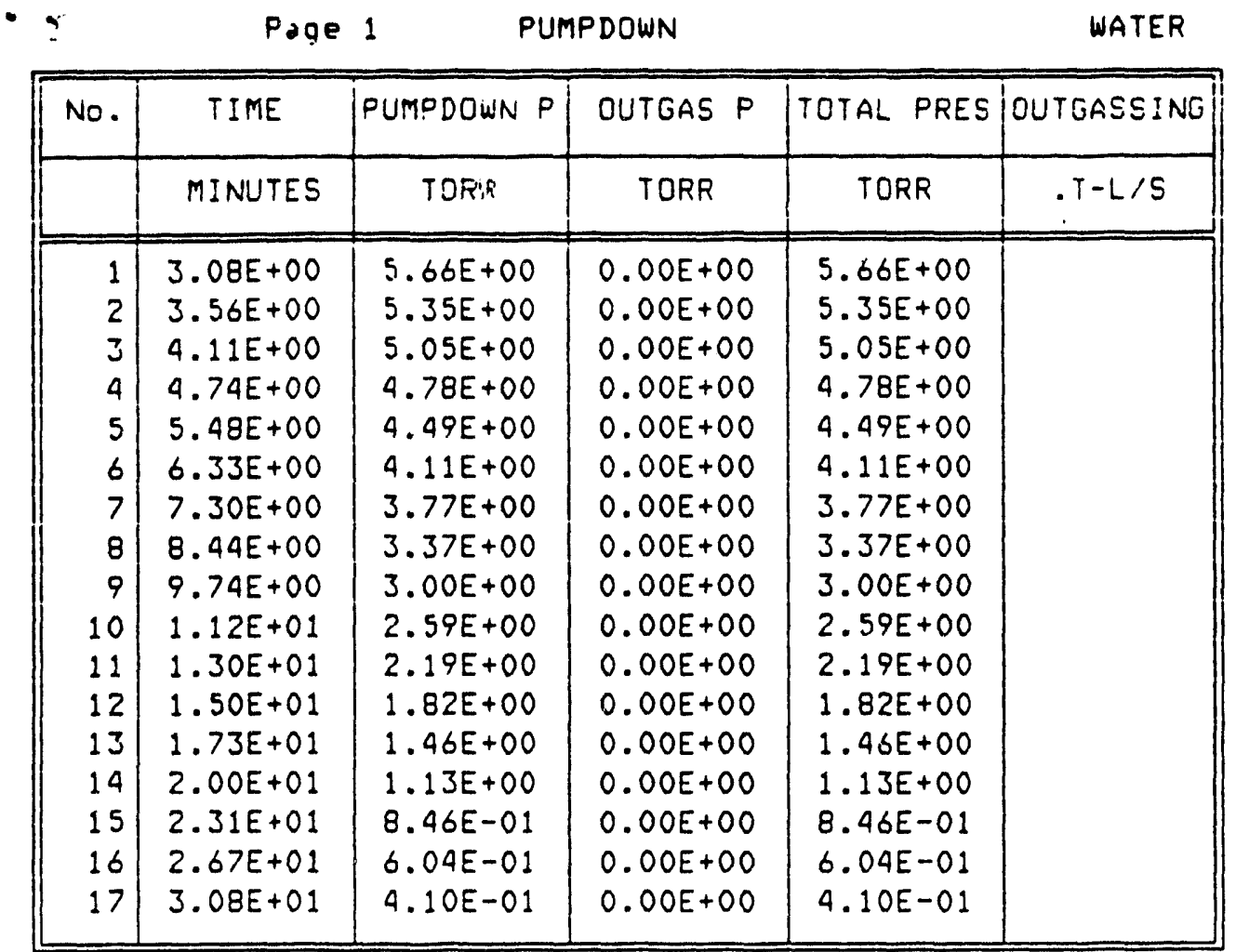

[Enter]-Next Page

[Esc]-Exit

Page 2

PUMPDOWN

WATER

\begin{tabular}{|c|c|c|c|c|c|}
\hline NO. & TIME & PUMPDOWN P & OUTGAS P & TOTAL PRES & OUTGASSING \\
\hline & MINUTES & TORR & TORR & TORR & $. T-L / S$ \\
\hline 18 & $3.56 E+01$ & $2.62 E-01$ & $0.00 E+00$ & $2.62 E-01$ & \\
19 & $4.11 E+01$ & $1.58 E-01$ & $0.00 E+00$ & $1.58 E-01$ & \\
20 & $4.74 E+01$ & $8.76 E-02$ & $0.00 E+00$ & $8.76 E-02$ & \\
21 & $5.48 E+01$ & $4.48 E-02$ & $0.00 E+00$ & $4.48 E-02$ & \\
22 & $6.33 E+01$ & $2.09 E-02$ & $0.00 E+00$ & $2.09 E-02$ & \\
23 & $7.30 E+01$ & $8.96 E-03$ & $0.00 E+00$ & $8.96 E-03$ & \\
24 & $8.44 E+01$ & $3.59 E-03$ & $0.00 E+00$ & $3.59 E-03$ & \\
25 & $9.74 E+01$ & $1.41 E-03$ & $0.00 E+00$ & $1.41 E-03$ & \\
26 & $1.12 E+02$ & $5.88 E-04$ & $0.00 E+00$ & $5.88 E-04$ & \\
27 & $1.30 E+02$ & $2.68 E-04$ & $0.00 E+00$ & $2.68 E-04$ & \\
28 & $1.50 E+02$ & $1.32 E-04$ & $0.00 E+00$ & $1.32 E-04$ & \\
29 & $1.71 E+02$ & $2.83 E-10$ & $0.00 E+00$ & $2.83 E-10$ & \\
30 & $2.00 E+02$ & $0.00 E+00$ & $0.00 E+00$ & $0.00 E+00$ & \\
31 & $2.31 E+02$ & $0.00 E+00$ & $0.00 E+00$ & $0.00 E+00$ & \\
32 & $2.67 E+02$ & $0.00 E+00$ & $0.00 E+00$ & $0.00 E+00$ & \\
33 & $3.08 E+02$ & $0.00 E+00$ & $0.00 E+00$ & $0.00 E+00$ & \\
34 & $3.56 E+02$ & $0.00 E+00$ & $0.00 E+00$ & $0.00 E+00$ & \\
\hline
\end{tabular}

[Enter]-Next Page

[Esc]-Exit 

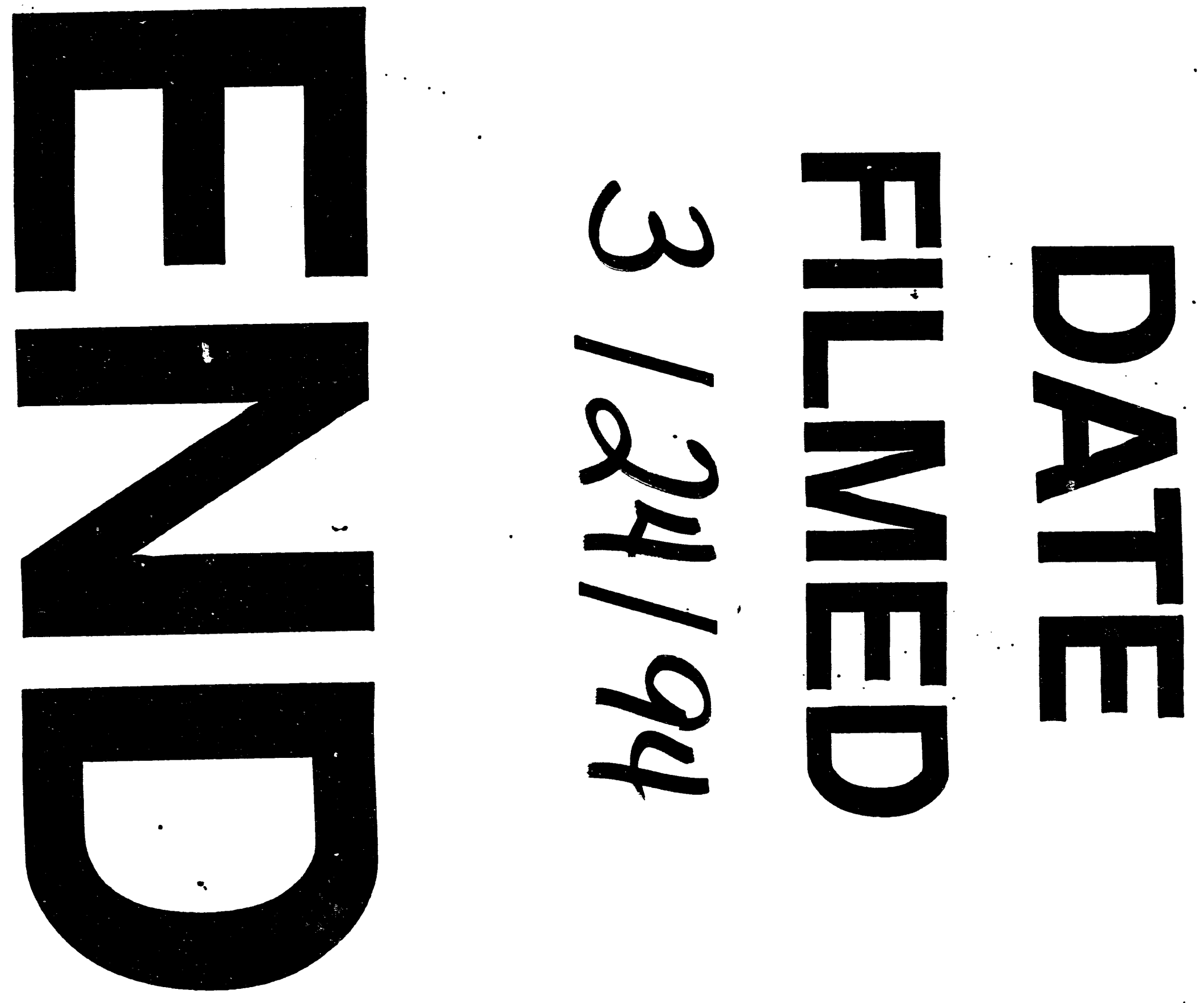


$$
-
$$

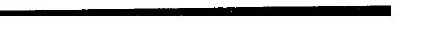

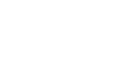

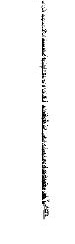

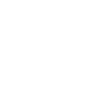

